\title{
Dynamics of galaxies with triaxial haloes
}

\author{
A.A. El-Zant ${ }^{\mathrm{a}, \mathrm{b}}$ B. Hassler ${ }^{\mathrm{a}, \mathrm{c}}$ \\ a Astronomy Centre, University of Sussex, Brighton, BN1 9QH, UK \\ b Physics Department, Technion - Israel Institute of Technology, Haifa 32000, \\ Israel \\ ${ }^{\mathrm{c}}$ DESY, Hamburg, Germany
}

\begin{abstract}
We study the stability of trajectories near the disk plane of galaxy models with a triaxial dark matter halo component. We also examine the effect of weak discreteness noise, rapidly rotating bar perturbations and weak dissipation on these trajectories. The latter effect is studied both by adding a dissipative component to the force law and by using particle simulations. If the matter distribution is triaxial and has a constant density core, dissipation leads to inflow of material inside the core radius to the centre (since no non-self-intersecting closed orbits exist in the central areas). This leads to the formation of central masses which in turn destabilise the trajectories of any stars formed in these regions. In particular, even if the gas settles by dissipation into a flat disk, stars formed in that disk will later form a bulge like distribution the extent of which would be related to the core radius of the halo and the original asymmetry in the plane. The process of gas inflow is regulated by the fact that too large and condensed a central mass leads to the creation of stable closed loop orbits in the central area around which the gas can move. This would appear to stop the accumulation of central mass before it becomes large enough for rapid loss of halo triaxiality. It was found that weak discreteness noise can increase the fraction of such trajectories significantly and can therefore have important consequences for the modelling of galaxies. The addition of rapidly rotating bar perturbations also increases the degree of instability dramatically. So if bars can form in triaxial haloes they are likely to be quickly destroyed leaving a bulge like structure behind (which may explain the absence of bars in surveys of high redshift galaxies). In they do survive their main effect on the gas dynamics is to create attractors other than the centre around which the gas can move. We discuss some possible consequences of the aforementioned effects. In particular, it is suggested that the halo core radius and initial asymmetry may be important in determining the relative disk-halo contribution to the rotation curve of a galaxy and hence its Hubble type.
\end{abstract}

Key words: galaxies: evolution - chaos - galaxies: haloes - instabilities - galaxies: kinematics and dynamics

PACS: 98.10.+z,95.10.Fh,98.62.Gq

Preprint submitted to Elsevier Preprint

20 September 2018 


\section{Introduction}

Numerical simulations of dissipationless collapse starting from cosmological initial conditions consistently predict triaxial final states for cold dark matter (CDM) halos (e.g., Dubinsky \& Carlberg 1991; Warren et al. 1992; Cole \& Lacey 1996). Considering the fact that collapsing spherical objects are unstable towards non-spherical perturbations (Lin, Mestel \& Shu 1965), and that violent relaxation is not completely effective in washing out initial asymmetries (Aarseth \& Binney 1978), these results are perhaps not that surprising. In fact, given the centrally concentrated radial density distribution of the resulting halos, the observed asymmetries should be considered as lower limits, since, for such structures, many of the trajectories will be chaotic. As these trajectories explore their phase space, the equilibrium figure will tend to relax towards more symmetric shape. This process would be expected to be strongly amplified by discreteness noise (Merritt \& Vallury 1996 and Section 4.2 of the present paper) which is much larger in numerical simulations than in CDM system.

Using parameters obtained from numerical simulations (of Dubinsky \& Carlberg 1991), Kuijken \& Tremaine (1991) estimated that a disk equipotential axis ratio of $\sim 0.9$ can be inferred. Since then, there has been a number of papers suggesting that such a value is incompatible with observations. These include arguments that the scatter in the Tully-Fisher relation would be too large (Franx \& de Zeeuw 1992). As was pointed out by Rix \& Zaritsky (1995) however, the fact that the Tully-Fisher relationship is used for distance measurement might make the sample used particularly biased against galaxies with large-scale distortions. These authors use the shapes of the isodensity contours from near-infrared images of external galaxies to obtain an axis ratio of $\sim 0.95$ for the disk isopotentials — this is consistent with a halo potential axis ratio $\sim 0.9$, if the disk and halo contribute more or less equally to the potential. Another argument for axisymmetric haloes makes use of the velocity dispersion of gas clouds in the Milky Way. From such considerations Blitz \& Spergel (1991) deduce that the potential axis ratio is near 1 (axisymmetric) unless the Sun lies near a symmetry axis of the potential. Kuijken \& Tremaine (1994), on the other hand, study in detail both local and global photometric and kinematic properties of our galaxy, and deduce that the Sun lies near the minor axis of the Milky Way (give or take 10 degrees), and that the data is consistent with a disk equipotential axis ratio of $\sim 0.9$ (meaning a halo potential axis ratio nearer to 0.8). Less controversial are measurements of the flatness of the halo. These can be obtained from observations of polar ring galaxies. They suggest a density axis ratio of about a half (e.g., Sackett 
\& Sparke 1990; Sackett et al. 1994). In general, therefore, one should expect the dark halos of galaxies to be very aspherical - in contrast to how they are usually modelled in simple treatments.

Whatever the shapes of haloes today, one may like to speculate about their evolution towards the present state and their influence on the early dynamics of disk galaxies. The fact that the flatness of haloes appears to be larger than the asymmetries in the disk plane, and that some studies constrain this asymmetry to be rather small, led some investigators to suggest that the dissipational formation of a disk in the centre of a triaxial halo causes the latter to evolve to more oblate nearly axisymmetric state: dissipation might lead to the formation of central concentration, which in turn might destroy the triaxiality (we consider this in detail later in this paper). However this is expected to be a slow process which should occur over many dynamical times. Indeed, triaxial figures of equilibrium with mild central density cusps have been successfully built in a self consistent manner (Merritt \& Fridman 1996: see also Schwarschild 1993 for an application to the singular logarithmic potential), and numerical simulations seem to show that there is a threshold for the ratio of the central mass to that of the triaxial figure ( $\sim$ a few percent) below which rapid loss of triaxiality does not occur (Merritt \& Quinlan 1997).

Studies of dissipational galaxy formation (e.g., Katz \& Gunn 1991) appear to contradict this. It is found that, in the presence of dissipation, the resulting haloes become nearly axisymmetric, and that this happened in the initial formation stages. However, modelling dissipational galaxy formation is not an exact science since the exact role and relative importance of the various hydrodynamic and thermodynamic effects is still unclear. Even more difficult to predict is the effect of star formation and the accompanying energy feedback. These effects, if underestimated, can cause over-dissipation leading to the buildup of central concentrations far greater than what can be realistically expected. In addition, the small number of particles used by Katz \& Gunn (about 4000), means that the discreteness noise is large. As mentioned in the opening paragraph, this can lead to artificial relaxation. A more controlled study was undertaken by Dubinsky (1994) who considers the slow growth of a Kuzmin Toomre disk initially placed at the centre of a live halo and the motion of which is evolved as a particle in the simulation. His conclusion is that, in the presence of the disk, the halo orbits change adiabatically, in a way as to cause the halo to become more oblate and less asymmetric in the disk plane. Nevertheless, the halo retains some of its triaxiality.

Indirect evidence that some disk galaxies might have had a triaxial halo at one point in their history but may have evolved towards axisymmetry relates to how one interprets the counter-rotating galactic disks that are observed (particularly the case of the S0 galaxy NGC 4550: Rubin et al. 1992; Rix et al. 1992). According to Evans \& Collett (1994), these symmetric populations 
result from stars which were on box orbits when the potential was triaxial, and follow loop orbits when the potential is axisymmetric. The stars on the box orbits in the original triaxial potential are either formed on these eccentric orbits or subsequently "heated" into box orbits. In fact, if the inner parts of galaxies are non-axisymmetric and are dominated by a nearly harmonic constant density core, it would be natural for stars confined within that core to move on box orbits. Indeed, the high velocity dispersions found in the inner regions of galactic disks (Lewis \& Freeman 1989) suggest that a large fraction of stars in these regions either once were or still are on box orbits. Unless the harmonic core is very large, this population is likely to be confined to the inner few kpc. Moreover, we shall see in this paper, in the presence of a central mass, this group of stars can be transformed into a bulge population. This may explain why large scale counter-rotating disks are not commonly observed (Kuijken et al. 1996).

The work of Dubinsky (1994) and Evans \& Collett (1994) are examples of the evolutionary effects that disks can induce on galactic halos and vice-versa. The two effects described above however depend on the properties of the regular orbits in the potential: how they can be arranged in different ways. A richer variety of interesting phenomena are related to changes in the qualitative structure of orbits - that is the transition of orbits from regular to chaotic and vice-versa. In this case, a whole set of new phenomena may appear. Strongly chaotic orbits (visiting most of their allowed energy subspace) will usually have a time averaged density in the 3 dimensional configuration space that is much more round and isotropic than the underlying density distribution. The existence of a large number of these in a certain potential therefore implies that the velocity and density fields will evolve towards more isotropic distributions. An example of this effect is now well documented in the case of galactic bars. When a central mass is present, the once regular trajectories trapped around the $x_{1}$ closed periodic orbits (which support the asymmetric structure of the bar) become chaotic as the latter are destabilised - leading to the dissolution of the bar and the growth of bulge like structures (e.g., Hasan et al. 1993; Pfenniger \& Friedli 1991; Friedli \& Benz 1993; Norman et al. 1995). Similar effects are also thought to be important in the evolution of elliptical galaxies, where it is thought that the presence of a central mass or a significant density cusp causes these galaxies to lose their triaxiality (Norman et al. 1985; Gerhard \& Binney 1985; Merritt \& Fridman 1996; Merritt \& Quinlan 1997).

We wish here to study similar effects in models of disk galaxies with triaxial halos. In this situation, both processes described above may act. One expects that the effect of chaotic behaviour will modify the disk stellar distribution, while at the same time causing the halo to become more axisymmetric. The model characteristics that are expected to be important in the determining the extent of chaotic behaviour (the degree of asymmetry and central concentration) are the same in this new context (Section 3.2 and 4.1), though the 
interpretations may be different. In addition, also interesting here are situations in which both a non-rotating triaxial halo and a rapidly rotating bar are present (Section 4.3). We will also be examining the effect of weak discreteness noise (Section 4.2) and weak dissipation (Section 4.4 and 4.5). We will find that the latter property has the interesting effect of triggering significant inflow of material to the central regions of our models.

We will be concerned with the stability of motion near the disk plane of fully three dimensional models. By symmetry, all orbits have to pass at least once by the disk plane. Trajectories that remain confined near the disk plane however are usually regular. Those exploring the full configuration space bounded by the (approximately ellipsoidal) zero velocity surface are strongly chaotic (an exception was found in the case of models with rotating bars, but here one is mainly interested in the bars' effect on orbits in the plane in any case). Thus, testing for vertical stability near the disk plane, at the same time gives an idea of the fraction of chaotic orbits in a given model, while also determining the fraction of trajectories that would be vertically unstable from an initial distribution started near the disk plane. These two related effects determine the plausibility of maintaining a triaxial halo, and a flat distribution of stars in the inner hotter areas of the disk. While a search confined to the vicinity of the disk plane is obviously not exhaustive, it should be representative since the three dimensional regular orbits are usually parented by those in the symmetry planes (e.g., Binney \& Tremaine 1987 (BT)) and strongly chaotic orbits should pass at any given point in the disk plane with a vertical velocity close to zero (Poincaré recurrence theorem). On the other hand, the reduction in the number of phase space parameters to be searched, can be used in exploring the extensive set of models which we will now describe.

\section{The models}

\subsection{The halo}

A well known simple expression for a triaxial halo with an azimuthally averaged rotation curve that flattens at large radii is

$$
\Phi_{H}=\frac{1}{2} v_{0}^{2} \log \left(R_{0}^{2}+x^{2}+p y^{2}+q z^{2}\right)
$$

where $v_{0}$ is the maximum rotation velocity and $R_{0}$ is the radius of a core where the potential is nearly harmonic. The parameters $p$ and $q$ are related to the potential axis ratios by $p=(a / b)^{2}$ and $q=(a / c)^{2}$, with $a>b>c$ for a triaxial halo. 
We will usually fix $v_{0}$ to a value close to $200 \mathrm{Km} / \mathrm{s}$. This is consistent with the value attributed to the halo contribution in the Schmidt (1985) model of the Galaxy (see also van der Kruit 1986). In this model, the value for $R_{0}$ is found to be about $2.8 \mathrm{kpc}$. $R_{0}$ also can be determined by decomposing the rotation curve into disk, halo and bulge contributions using the "maximum disk" procedure (see, e.g., van der Kruit 1989). It is still not clear whether real galaxies satisfy this assumption, although some authors do argue in that direction (e.g., Freeman 1993). In this decomposition method $R_{0}$ can be very large (Verdes-Montenegro et al. 1996 and Botteno 1996 found it to be 11 and $30 \mathrm{kpc}$ respectively). By spanning a range of possible core radii however (not only those consistent with maximum disk), it is possible to find smaller values. For example for NGC 2903, Hofner \& Sparke (1994) find values between 2.4 to $8 \mathrm{kpc}$. Numerical simulations based on CDM models on the other hand predict no core radius at all, but a density increasing as $1 / r$ in the central areas. It has been argued however (Flores et al. 1993) that small (or zero) values for the core radius would mean that the density distribution is halo dominated even in the inner regions of bright galaxies, making it difficult to accommodate for the luminous contributions of galactic disks (which can explain the observed rotation in the inner regions of such galaxies: e.g., Persic, Salucci \& Stel 1996; Tully \& Verheijen 1997. See however Navarro 1996). In addition, dynamical arguments concerning the braking of rotating bars, suggest that long lived bars must reside in nearly maximal disk density distributions (Debattista \& Sellwood 1996). Moreover, for low surface brightness galaxies, where the halo does indeed dominate over all radii, such centrally concentrated halo densities as those predicted by simulations would be incompatible with the observed rotation curves (Flores \& Primack 1994). Thus, despite the success of CDM models in reproducing other observed features of dark haloes (like correlations between total mass and scale lengths), observational evidence suggests that dark haloes should have substantial harmonic cores (Burkert 1997; Persic, Salucci \& Stel 1996).

The importance of a harmonic core lies in the fact that within it only box orbits can exist. Dissipation therefore implies that the only stable attractor is the centre (since there will be no stable closed loop orbits to oscillate against: Section 4.4.1). This will imply that, as a disk settles down in such a potential, there will be a building up of central mass concentration, which in turn would destabilise both the halo box orbits and those stars formed in the plane where the disk is settling which, at least in the central areas, will also mostly have to be on box orbits. On the other hand, in the case when the core radius is very small, stable loop orbits exist everywhere, and gas can settle around them. Nevertheless, stars born in rapidly evolving non-equilibrium situations, or subsequently heated by discreteness effects (e.g., encounters with gas clouds) from their nearly circular orbits, can have eccentric trajectories - and in cusped potentials these are usually chaotic. The very high velocity dispersion found for old stars in the inner disk by Lewis \& Freeman (1989) suggests that one 
of the above scenarios should hold.

In our models we have tried values between $R_{0}=2 \mathrm{kpc}$ and $R_{0}=6 \mathrm{kpc}$ which span a range of observationally allowable values. We also did some runs with $R_{0} \sim 0$ with and axisymmetric halos — on the assumption that such highly cusped structures would not remain triaxial.

The concept of triaxiality can be made more formal by using the "triaxiality parameter" (Warren et al. 1992; Franx et al. 1991)

$$
T=\frac{a^{2}-b^{2}}{a^{2}-c^{2}} .
$$

If $T \sim 0$ then the halo figure shape is nearly oblate, while when $T \sim 1$ the halo it is essentially prolate. We choose two values for the triaxiality parameter in our models: $T=0.85$, corresponding to a strongly prolate shape close to the most probable values found by Warren et al. (1992) for the equidensities, and $T=0.65$ - close to the value found by Dubinsky (1994) for his equidensities (we assume here that the triaxiality of the potential distribution follows that of the density, at least in the case when the asymmetry is not too large). Although a value of $T=0.65$ still describes a shape that is more prolate than oblate, we will refer to halos that have $T=0.65$ as "oblate" to distinguish them from those with $T=0.85$ which will be referred to as "prolate".

\subsection{The disk}

Although an exponential disk is most realistic in modelling the galactic disk density distribution, we have decided to use a Miyamoto-Nagai (Miyamoto \& Nagai 1975) disk because it enables one to model simultaneously a bulge and disk distribution without any additional components. Is is also easily handled computationally with the force obtained in closed form by taking the derivatives of the potential given by

$$
\Phi_{D}=-\frac{G M_{D}}{\sqrt{x^{2}+y^{2}+\left(a_{d}+\sqrt{b_{d}^{2}+z^{2}}\right)^{2}}},
$$

where $G M_{D}$ is the mass of the disk in units in which the gravitational constant is unity. The parameters $a_{d}$ and $b_{d}$ determine the central concentration and the flatness of the disks respectively. In our models the mass of the disk was usually about $3 \times 10^{10}$ or $6 \times 10^{10}$ solar masses, and the scale length $a_{d}$ was usually taken to be either $1.5 \mathrm{kpc}$ or $3 \mathrm{kpc}$ while the scale height $b_{d}$ was usually either $0.5 \mathrm{kpc}$ or $1 \mathrm{kpc}$. 


\begin{tabular}{l|l|l|r|r|l}
\hline Model & $V_{0}$ & $R_{0}$ & $G M_{D}$ & $a_{d}$ & $b_{d}$ \\
\hline Model 1 & 0.2 & 2 & 0.15 & 3 & 1 \\
\hline Model 2 & 0.19 & 6 & 0.3 & 3 & 1 \\
\hline Model 3 & 0.205 & 6 & 0.15 & 1.5 & 0.5 \\
\hline Model 4 & 0.19 & 6 & 0.3 & 7.8 & 0.2 \\
\hline Model 5 & 0.18 & 4 & 0.3 & 3 & 1 \\
\hline Model 6 & 0.1 & 4 & 0.6 & 3 & 1
\end{tabular}

Table 1

Parameters of the different disk-halo models that were studied. The first three models are discussed in detail in this paper

\subsection{Models with disk and halo}

The choice of simple analytic formulae for our potentials ensured that the force calculations are not CPU-time consuming. This enabled us to explore large parts of the parameter spaces. In Table 1 we list the different disk and halo parameters that were used. These are given in units of $\mathrm{kpc}, \mathrm{kpc} / \mathrm{Myr}$ and $G=1$ - which implies that the unit of mass is about $2 \times 10^{11} M_{\odot}$. Any numerical values for the different dynamical variables will be assumed throughout the rest of this paper to be expressed in terms of the above units unless it is explicitly stated otherwise.

We model a sequence of galaxies with different disk-halo contributions to the rotation curve: from those for which the halo dominates everywhere (e.g. Model 1), which is likely to be the case in low surface brightness galaxies, to others where this component dominates only in the outer region (e.g., Model 2 or Model 3). Clearly, in the latter case, the disk will have to be either more centrally concentrated or more massive. These two situations are represented by Models 3 and 2 respectively. The first case is a typical near maximal disk situation, while the second case represents a situation where both components are comparable (as suggested by Navarro 1996). The three first models are therefore representative of the range of objects of interest. Nothing qualitatively interesting arose from the examination of the other models and so they will not be discussed any further.

The rotation curves of the three chosen models are shown in Fig. 1. All model parameters were fixed so that the rotational velocity at $20 \mathrm{kpc}$ was $\sim 0.22$ kpc/Myr ( $220 \mathrm{~km} / \mathrm{s})$. Although according to Persic, Salucci \& Stel (1996) different galaxy types are characterised by different maximal rotational velocities, it facilitates the comparison of dynamical properties if all models have 

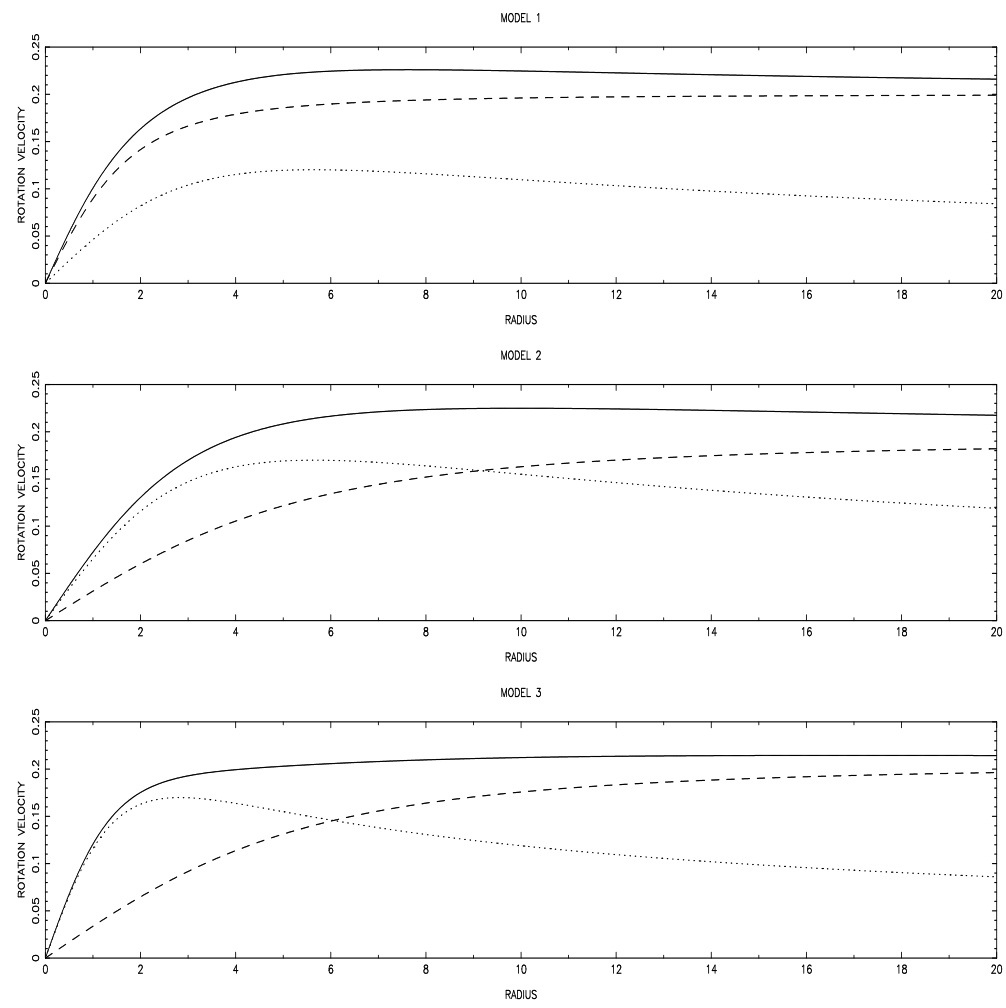

Fig. 1. Rotation curves of the three disk-halo models studied in this papers. The dotted lines correspond to disk contributions, the dashed lines to the halo contributions while the combined disk-halo rotation curves are shown by the solid lines

the same rotation speed at a given radius (and hence roughly the same total mass distribution and dynamical time-scale). In practice, the variation of total mass and mass to light ratios is rather small over a wide range of Hubble types, and the scatter in galaxy properties is such that it is easy to find galaxies of different types with the same maximal rotation speed (Roberts \& Haynes 1994).

To completely fix the system parameters, there remains now the task of determining the values taken by the potential axis ratio of the halo in the disk principal plane. This fixes $p$ and, since we have chosen two definite values for the triaxiality parameter $T$ (see Section 2.1), $q$ is then automatically fixed. This was done by looking at the axis ratios of the closed periodic orbits, which is the main observable measure of the total asymmetry in the disk-halo system that is produced by a given asymmetry in the halo (e.g., Rix 1995).

The variations of the axis ratio of the closed loops with radius for the three main models with halo potential axis ratio $b / a=0.7$ are shown in Fig. 2 , while the plots in Fig. 3 show the variation of this parameter as a function radius for Model 2 with potential axis ratios $b / a=0.9,0.8$. Clearly, in general, an axis ratio of $b / a=0.9$ is compatible with all current observational constraints (Section 1) on the eccentricity of galactic disks, an axis ratio of $b / a=0.8$ is 

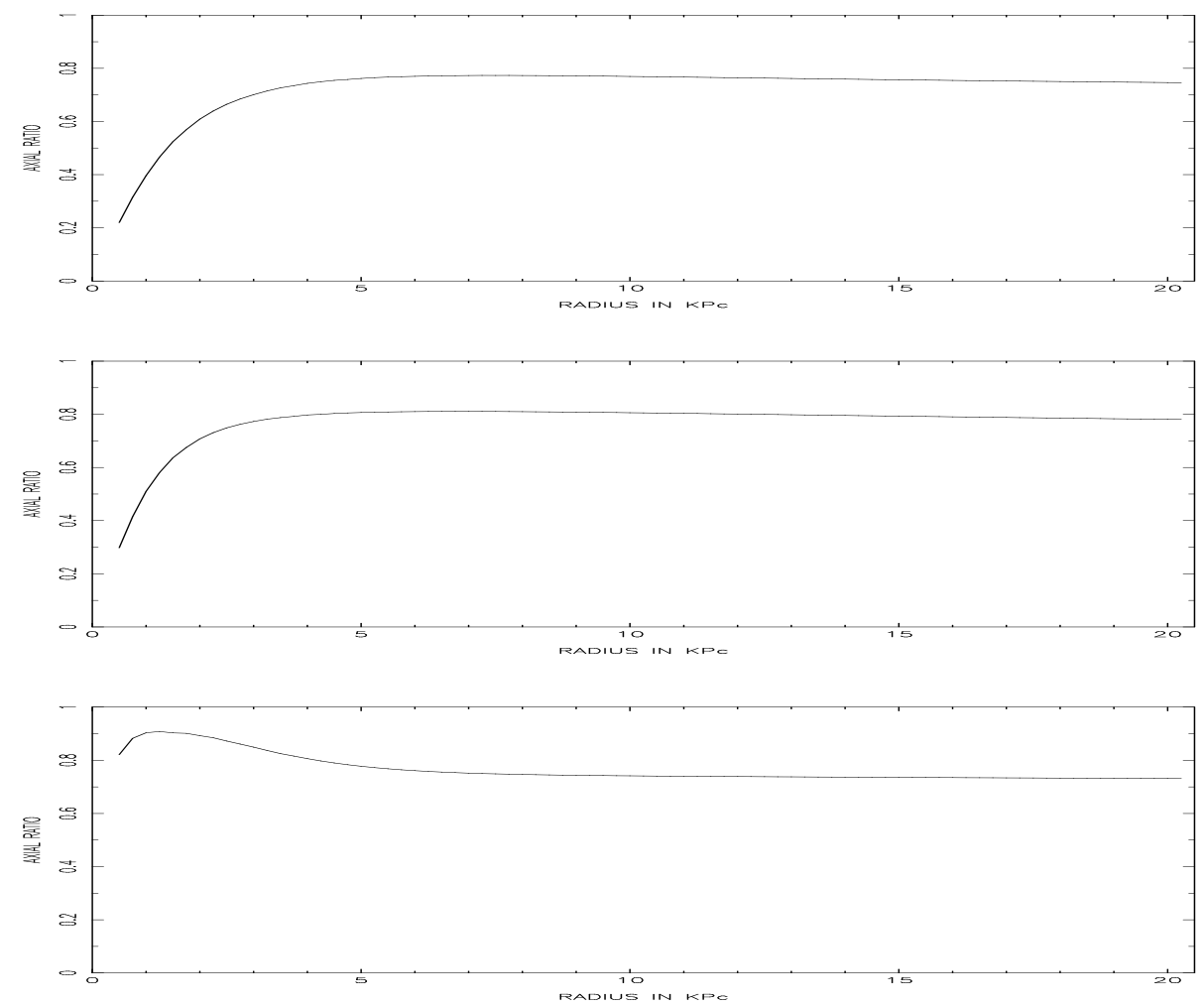

Fig. 2. Variation with radius of the axis ratios of the closed loop orbits in the disk planes of Model 1 (top), Model 2 (centre) and Model 3 (bottom) when the halo potential axis ratio in that plane is $b / a=0.7$
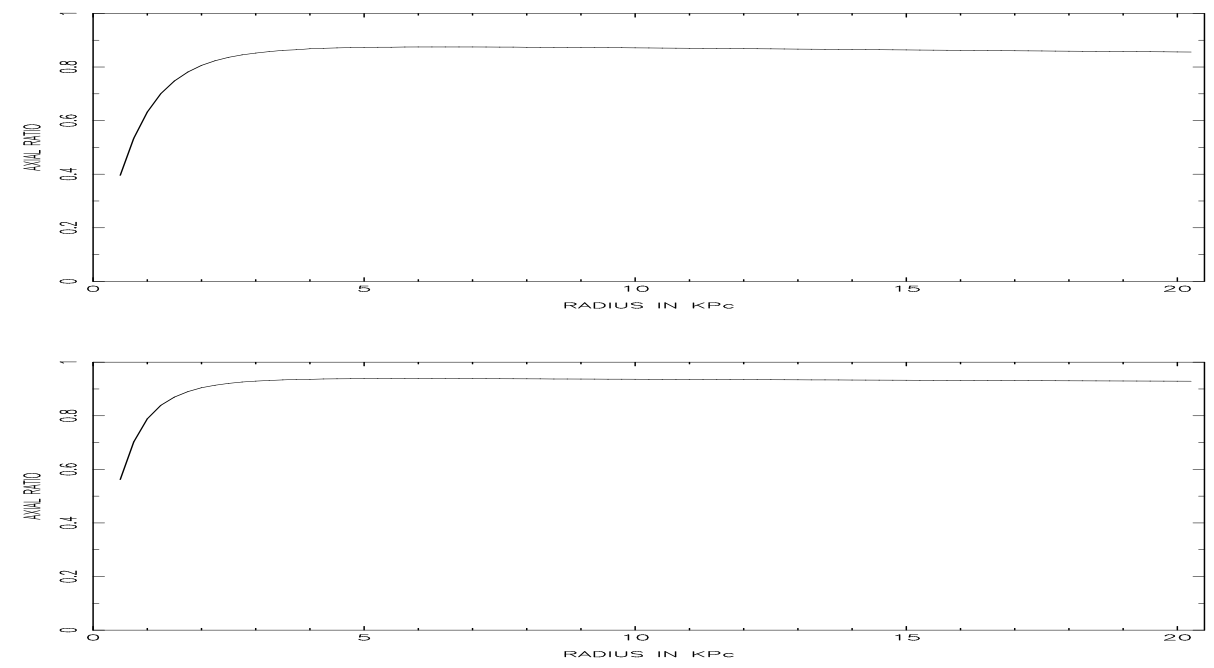

Fig. 3. Variation with radius of the axis ratios of the closed loop orbits in the plane of Model 2 when the potential axis ratio in that plane is $b / a=0.8$ (top) and when it takes the value $b / a=0.9$ (bottom) 
more or less compatible with the results of Kuijken \& Tremaine $(1991,1994)$, while an axis ratio of $b / a=0.7$ is more eccentric than what is compatible with current observations (except perhaps for certain regions of Model 3) it is not ruled out however that such eccentricities were present at earlier stages of galaxy evolution. For the prolate case $(T=0.85)$ all three values of $b / a=(0.9,0.8,0.7)$ were used, for the oblate $(T=0.65)$ case only the values 0.9 and 0.8 were used. In the latter case, the value 0.7 was not used because it corresponds to a density distribution that was seriously dented and because one expects the oblate shapes, which form after dissipation is complete, to be less asymmetric (Dubinsky 1994).

Although the logarithmic halo is known to have wild dents in its density distribution when the asymmetry is too great, the models we have chosen have a well behaved density distribution that is positive everywhere. The conditions for this to be the case as well as equidensity contours for some of the models studied here are given in the Appendix.

\subsection{Central mass}

This was taken to be a Plummer sphere with core radius $b_{p}=0.01 \mathrm{kpc}$. The potential for this object is

$$
\Phi_{C}=-\frac{G M_{C}}{\sqrt{b_{p}^{2}+x^{2}+y^{2}+z^{2}}} .
$$

Many values of the mass $G M_{C}$ were experimented with but only runs corresponding to models with no central mass, or with a "weak" central mass of $G M_{C}=0.0005$ (about $10^{8} M_{\odot}$ which amounts to $\sim 0.05 \%$ the total mass at 20 kpc), or a "strong" central mass of $G M_{C}=0.01$ (which is about $2 \times 10^{9} M_{\odot}$, $\sim 1 \%$ the total mass at $20 \mathrm{kpc}$ ) are discussed in detail since they convey the essential results.

\subsection{Naming disk-halo-central mass systems}

Although we have tried to keep this to a minimum, the following nomenclature was sometimes used in referring to a certain run. If the model used was Model 1 for example we refer to it as M1, this is followed by either the letter $\mathrm{P}$ (for prolate models) or $\mathrm{O}$ (for oblate ones) and then by one of the following numbers 1 (for spherical halo) or by 9,8 or 7 for halo potential axis ratio $b / a=0.9,0.8,0.7$. If no central mass was present, this is followed by $\mathrm{C} 1$, if $G M_{C}=0.0005$ it is followed by $\mathrm{C} 2$ while if $G M_{C}=0.01$ this is followed by C3. 
Thus a complete description of a run is referred to by a series of symbols like M1P7C2 (which in this case describes a system with the general characteristics of Model 1 and when the halo is prolate, $b / a=0.7$ and $G M_{C}=0.0005$ ). If a disk is not present in the given system then -D is added (e.g., M1-DP7C2).

\subsection{Bars}

In some runs a rapidly rotating barred perturbation was added in the disk plane. Two types of bar models were considered: a Ferrers ellipsoid of order two (BT; Pfenniger 1984a) and a homogeneous rectangular box (Macmillan 1958). The density of the Ferrers bar is constant along contours given by

$$
m^{2}=x^{2} / a_{b}^{2}+y^{2} / b_{b}^{2}+z^{2} / c_{b}^{2}
$$

where $a_{b}>b_{b}>c_{b}$ are the semi-axes of the density distribution. Inside the mass distribution $(m<1)$ the density varies as

$$
\rho=\rho_{c}\left(1-m^{2}\right)^{2}
$$

where the central density $\rho_{c}$ is determined by the total mass of the bar $G M_{B}$. The density is zero for $m \geq 1$.

The semi-axes are taken as $a=6, b=1.5$ and $c=0.5$ (as in Pfenniger 1984a). The rotation speed of the bars was always chosen so that the bar major axis coincided with the distance from the center to the Lagrangian point $\mathrm{L}_{1}$ near corotation (calculated while assuming an axisymmetric halo and not including the bar's own contribution to the potential). This corresponded to an angular speed of about $0.0372,0.0361,0.0343$ in Models 1,2 and 3 respectively. Unless otherwise stated, the bar mass was taken to be $G M_{B}=0.02\left(\sim 4 \times 10^{9}\right)$, which amounts to $13 \%$ of the disk's total mass in Models 1 and 3 and $6.5 \%$ of that mass in Model 2.

\section{$3 \quad$ Stability of periodic orbits}

\subsection{Order and chaos in triaxial galaxy models and the role of periodic solu- tions}

It is well known (e.g., LL; BT) that the stability properties of periodic orbits are of central importance in determining the qualitative phase space structure 
of Hamiltonian systems. For regular trajectories are parented by stable periodic orbits while at least some periodic orbits have to be unstable for a system to support chaotic trajectories (i.e., be non-integrable). If periodic solutions form a dense set of unstable trajectories, then a system can be said to be uniformly hyperbolic and almost all trajectories are chaotic. Separable systems on the other hand have a finite number of stable and unstable closed orbits with the remainder being marginally stable. General systems will lie on the transition between these two extremes (for an interesting original perspective on the nature of this transition see Aubry 1985).

It is intuitively clear from the above remarks that as the density of periodic solution increases (for a given period length), it is less likely that we have the remarkable situation of a separable system where all but a finite number of closed solutions are marginally stable. Indeed, it can be shown (e.g., Gutzwiller 1990) that the number of periodic solutions in a chaotic system increases much faster (exponentially) with the period than that in a regular system. The density of periodic orbits will depend on the nonlinearity of a given system. For example, in a linear system the frequencies of the independent oscillations do not depend on the amplitudes. In this case therefore, the ratios of the oscillation frequencies in the different degrees of freedom are constant and are likely to be irrational. Therefore, without getting into the details, one can guess that the more the oscillation frequencies vary with amplitude, the denser is the set of resonant solutions and the more likely the system is to be chaotic. In particular, inside the nearly harmonic cores of systems studied here, we expect most orbits to be regular and parented by the axial orbits (e.g., BT). Instability in this "box" orbit family will appear only when nonlinearity starts becoming unimportant and initially around the lower order resonances (in particular we will see that the 2:1 resonance is highly effective). The addition of a central mass causes the oscillation frequencies along the principal axes to vary strongly with amplitude. This creates a dense set of unstable periodic orbits (represented by gaps along an axial orbit) and the axial orbit is destabilised almost everywhere. When a sufficiently strong central mass is added, the boxes, which are parented by the axial orbits, are therefore destroyed and replaced by other families of orbits.

Even in a situation where the frequencies of oscillations in the different degrees of freedom vary (may be even strongly) with amplitude it is possible that their ratios remain constant. For example, in power law potentials, the ratio of the frequency of circular motion to that of a perturbation normal to it (in the plane and in the vertical direction) is constant. In such a situation one again expects the trajectories parented by the stable closed nearly circular orbits to be regular quasiperiodic. In potentials with a harmonic core and which are superpositions of two power law terms (as is the case with our disk-halo systems) the scale free nature of the potential is destroyed and the frequency ratios are only nearly constant. However these will vary slowly 
Fig. 4. Poincaré maps of the ensemble of orbits in the disk plane of Model 1 when $b / a=0.8$. Initial conditions are given by Equations (12) to (16) (except that in the disk plane $z=0$ for all $i$ and $j$ ). Three different values for the central mass are chosen: $G M_{C}=0.0$ (top), $G M_{C}=0.0005$ (centre) and $G M_{C}=0.01$ (bottom). THIS FIGURE WAS TOO LARGE TO BE INCLUDED

enough for the above statement to be essentially true - especially far outside the core (precisely where motion on loop orbits is predominant). Even more generally, the frequency ratios of nearly circular orbits do not vary much with radius for any potential in which the density decreases outwards (e.g., BT) and therefore this situation is likely to be representative of most nonrotating triaxial potentials. The only way one breaks this symmetry is by adding a rotating perturbation - in which case it is possible to have a concentration of resonances and instability in the nearly circular $x_{1}$ orbit family — an effect which is again enhanced by the presence of a central mass concentration (see Pfenniger \& Norman 1990, especially the appendix).

Finally, for a system to support chaotic orbits, it cannot have too much symmetry. This can be either trivial symmetry as in axisymmetry leading to angular momentum conservation or more subtle symmetries in special integrable potentials. For a generic potential that contains no special symmetries one will expect that the more asymmetric the potential (as measured by the flatness and the triaxiality parameter), the further it will be from being integrable. In the case when the potential is inherently time dependent, time symmetry may also be broken and the Hamiltonian may not be conserved. In general it will be the combination of the factors mentioned above that will determine how widespread the chaotic behaviour is in any one of our models.

We illustrate these ideas with $y-\dot{y}$ Poincaré maps of trajectories in the $x-y$ plane of Model 1. The initial conditions chosen are those described in Section 4.1. These span a whole range of energies. Since Poincaré maps however are most effective when taken at definite energies (in that case, for example, the projections of different KAM tori do not overlap), we group the various orbits into six different energy levels. We then displace the $y$ coordinates of the orbits according to the energy interval they lie in: orbits in the first (lowest energy) interval do not have their coordinates displaced while orbits in the subsequent groups have their coordinates displaced by $-5 \mathrm{kpc},-11 \mathrm{kpc},-20$ $\mathrm{kpc},-34 \mathrm{kpc}$ or $-50 \mathrm{kpc}$ respectively. We vary the central mass while keeping the axis ratio of the halo constant with a value $b / a=0.8$. The integrations are done for $50000 \mathrm{Myr}$.

The results for central masses of $G M_{C}=0, G M_{C}=0.0005$ and $G M_{C}=0.01$ are shown in Fig. 4. When no central mass is present, most orbits belong to either the box or the loop orbit families. In the first energy level (nearest to the centre) all orbits are box orbits while at the second, third, and fourth energy levels the loop orbits start appearing in progressively larger fractions. 
Fig. 5. First two energy levels of Poincaré maps which are similar in all respects to those shown in Fig. 4 except that the following values for the central mass are adopted. $G M_{C}=0$ (top left), $G M_{C}=1 \times 10^{-6}$ (top right), $G M_{C}=1 \times 10^{-5}$ (centre left), $G M_{C}=5 \times 10^{-5}$ (centre right), $G M_{C}=0.0001$ (bottom left) and $G M_{C}=0.0005$ (bottom right). THIS FIGURE WAS TOO LARGE TO BE INCLUDED

The last two energy levels, contain a few higher order islands corresponding to stable "boxlet" and "looplet" orbits (see ,e.g., Schwarzschild 1993 and the references therein) and the first appearance of weakly chaotic trajectories.

A central mass of 0.0005 leads to the destruction of all box orbits. Most resulting chaotic orbits are "strongly stochastic" — not venturing only in the areas where the numerous higher order islands are present. Increasing the central mass further to 0.01 destabilizes many of these islands however and the chaotic orbits in these regions are free to roam through a still larger area of the phase space. This is especially so in the inner regions where the nonlinearity of the potential is strongest. In the outer regions some regular trajectories are found. These are parented either by higher order periodic orbits or by the closed loops (which remain regular since there is no rotating perturbation).

A more detailed view is provided in Fig. 5 where we have reproduced the Poincaré maps of the first two energy levels for a series of central mass concentrations of values up to $G M_{C}=0.0005$. It is clear that a central mass $G M_{C}=1 \times 10^{-5}$ (which is about $10^{-5}$ the mass of the system at $20 \mathrm{kpc}$ ) is already sufficient to destroy all box orbits in the central regions (although in that case most are still replaced by regular boxlets). Although by $G M_{C}=0.0005$ most trajectories in the inner region form part of a connected 'stochastic sea', significant remaining structure is still evident. Over shorter time-scales this structure is much more evident but its destruction is often speeded up by additional perturbations. It is therefore important in determining a system's response to these. We will discuss this in a little more detail in Section 4.4.

\subsection{Statistical stability properties of periodic orbits in disk halo models}

\subsubsection{Method}

A periodic orbit is an orbit whose trajectory returns to exactly the same point in phase space and appears as a set of isolated points on a Poincaré map. Therefore, if one chooses an arbitrary point $\mathbf{x}$ on a periodic orbit, under the mapping transformation $\mathbf{A}$ one has

$$
\mathbf{A}^{k}(\mathbf{x})=\mathbf{x}
$$


where $k$, the winding number, is an integer depending on the ratio of oscillation frequencies in the different degrees of freedom, and the plane chosen for the Poincaré mapping. An orbit with a given value of $k$ will return to its original location on the map after $k$ iterations. It is the low $k$ orbits which determine the basic phase space characteristics (e.g., LL).

We have made extensive searches for closed periodic orbits in the disk plane up to order four in $k$. For each orbit we checked the stability to perturbations in and normal to the disk plane. We did not follow in detail the main orbital families and their bifurcations. In a far from integrable system this is a fairly intricate task due to the large number of orbital families and accompanying bifurcations. It also misses "irregular" orbits (Barbanis \& Contopoulos 1995) which do not bifurcate from other families. The above procedure is especially complex when one has to deal with several bifurcation parameters - the flatness, the triaxiality, the energy and the central mass. In such a situation, it might seem that the best chance of obtaining a representative sample of the types of closed orbits populating phase space, is by undertaking a systematic search of that space. This search was made possible by the use of the globally convergent search technique (see below) for solving Eq. 7. Whereas in the standard method one finds the main families at one energy by taking a Poincaré map and then simply following their characteristic curves using local search routines, using a globally convergent routine means that one can search the whole phase space. This method is of course more time consuming and the grid of trial initial conditions is necessarily coarse. In addition, the routine may fail for some initial conditions. Nevertheless, due to the advantages stated above, we have found it preferable.

The initial conditions for the searches were chosen as follows. Five values of the energy were chosen. These corresponded to the values of the potential energies on the $x$-axis at 1 and $21 \mathrm{kpc}$ and three intermediate values sampled at regular intervals. The initial positions for the searches — which are taken along the $x$-axis - are chosen to be between 1 and $15 \mathrm{kpc}$ (inclusive) and at intervals of $2 \mathrm{kpc}$. The initial $x$ velocities are then varied at intervals of 0.06 $\mathrm{kpc} / \mathrm{Myr}$. One stops incrementing the $x$ velocities when the corresponding $y$ velocities, which are obtained from

$$
\dot{y}=\sqrt{2(E-V)-(\dot{x})^{2}},
$$

become imaginary.

The number of iterations required by the routine to solve Eq. 7 of $n$ variables grows as $n^{2}$. It is therefore important to minimize the number of variables involved. On the Poincaré section on which the periodic solutions are searched there are three variables. It is possible to explicitly eliminate one more variable by using condition (8). However, during its search far from a root, the routine 
is likely to venture into regions of the phase space where this relation implies complex $\dot{y}$ causing frequent failure. An alternative strategy (implicitly using the conservation of energy) was therefore adopted. At each iteration, the $x$ coordinate was preset to a given value (the one from which the search originally started) and the search was conducted over the two velocity coordinates which were left to vary until the final values were identical to the original preset values (within a relative error of $\sim 10^{-10}$ ) for two successive iterations. Because energy is conserved during the integration and $y=0$ on the map, the $x$ coordinate is then automatically determined to within a sign. This is fixed by requiring the mapping to contain the condition of $x \geq 0$ in addition to the standard condition $\dot{y} \geq 0$ (the symmetry of the potential means that this constraint causes no loss of generality). This procedure of course means that the final energy of the periodic orbit is different from that of the initial search energy. It is usual however that this procedure finds the roots nearest to the search energy, so that a more or less uniform distribution of energies emerges. This however was not always found to be the case and some orbits were found that extended to very large radii.

Periodic orbits are located by finding zeros of the function

$$
\mathbf{F}(\mathbf{x})=\mathbf{A}^{k}(\mathbf{x})-\mathbf{x}
$$

For this purpose, we have used the globally convergent hybrid method of Powell (1968) as implemented in the NAG routine C05NBF. One starts from an initial guess to the solution which need not necessarily be close to an actual periodic orbit as in the Newton-Raphson method (e.g., Parker \& Chua 1989). This latter procedure is only applied after a scaled gradient minimizing technique has identified such a neighbourhood from which it is not likely to fail. It then converges quadratically in this neighbourhood (i.e., at each iteration, the number of digits to which the solution is accurate is doubled).

The linear stability of periodic orbits is determined by the derivatives of the map (7). In a Poincaré section, at constant energy, two coordinates are eliminated (which in our case happen to be $y$ and $\dot{y}$ ). For orbits in the symmetry plane of a galactic potential, the variational equations are decoupled in $x-\dot{x}$ and $z-\dot{z}$ independent components (since in that case mixed derivatives of the potential vanish: Hénon 1973). The stability of periodic orbits near the disk plane can then be expressed in terms of the stability parameters $b_{x}=\partial A_{x} / \partial x+\partial A_{\dot{x}} / \partial \dot{x}$ and $b_{z}=\partial A_{z} / \partial z+\partial A_{\dot{z}} / \partial \dot{z}$. These were approximated using

$$
\frac{\partial A_{i}}{\partial x_{j}}=\frac{A_{i}\left(x_{1}, \ldots, x_{j}+\Delta x_{j}, \ldots\right)-A_{i}\left(x_{1}, \ldots, x_{j}-\Delta x_{j}, \ldots\right)}{2 \Delta x_{j}}
$$



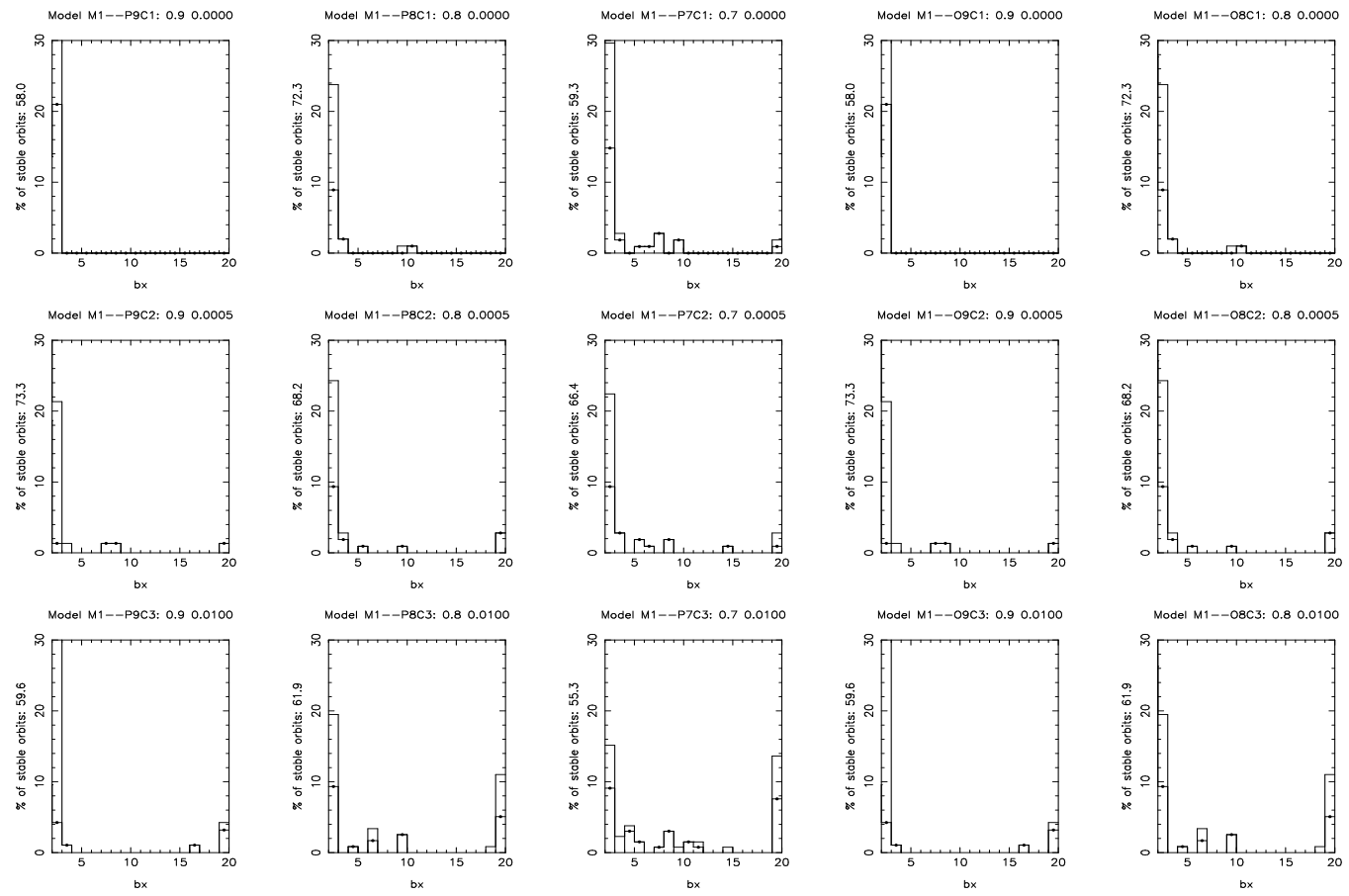

Fig. 6. Distribution of horizontal stability parameter $b_{x}$ for the periodic orbits of Model 1 of winding number up to 4 . The different runs are labeled according to the convention described in Section 2.5. Orbits with $\left\|b_{x}\right\| \leq 2.00005$ are labeled stable and the fraction of such orbits is indicated on each diagram

For each derivative, several values of the increment were tried (so as to ensure that the result did not sensitively depend on the increment, for as is well known, taking numerical derivatives is a numerically unstable operation which has to be performed with care) and an error estimate was derived. The increments were chosen so as to ensure that the relative error was less than $10^{-4}$.

In each of the models many hundreds of orbits were recovered but most were found to duplicate each other. After removing the duplicates one hundred or so orbits were found in each model. For these orbits, we bin the horizontal and vertical stability indices depending on their absolute values. The first bin contains all stable (and very close to stable) values $\left\|b_{x}\right\|,\left\|b_{z}\right\| \leq 2.0005$. The subsequent bins cover the interval from $\left\|b_{x}\right\|,\left\|b_{z}\right\|=2.0005$ to $\left\|b_{x}\right\|$ , $\left\|b_{z}\right\|=20.0$ in intervals of one (except for the second bin which covers the slightly smaller interval from values of 2.0005 to 3 ). The number of orbits is then normalized to a hundred.

\subsubsection{Results}

The resulting histograms for values of $b_{x}$ in Model 1 are shown in Fig. 7. As would be expected, since one is testing stability in the plane, the results are 
unaffected by the flatness of the halo and depend only on the asymmetry in the $x-y$ plane and on the existence and strength of the central mass. Also as expected from the discussion above (Section 3.1), the fraction of unstable orbits increases with both of these quantities. For as can be seen from this figure, the periodic orbits found for Model M1-P9C1 for example are all stable, or very close to being so (i.e, close to marginal stability). When a central mass $G M_{C}=0.0005$ is present, the fraction of stable periodic orbits actually increases. This is due to many previously marginally (un)stable orbits becoming stable. A corresponding number of orbits become highly unstable $\left(\left\|b_{x}\right\|>3\right)$. Increasing the central mass to $G M_{C}=0.01$ increases the number of unstable orbits - both the ones near marginal stability and ones with largest absolute values of $b_{x}$. This happens at the expense of the fraction of stable orbits which decreases through second order bifurcations.

The above effects are more pronounced for models with smaller values of $a / b$. However, there is always a significant fraction of stable periodic orbits. These include the closed loops and the boxlets which do not pass near the centre. It is interesting to note however that many of the most unstable orbits in some of the runs (e.g., M1-P8C3 and M1-P7C3) are tubelets which do not pass by the centre. This last result suggests that although one may think of the reason orbits in centrally concentrated potentials are unstable is that they are "scattered" by the central mass, it is more appropriate to see the central mass as introducing strong non-linearity which, in potentials without special symmetry, creates unstable closed orbits. General orbits are then repelled in phase space by the unstable periodic orbits destabilised by the non-linearity. The reason that loop orbits remain regular is that (for reasons outlined in Section 3.1) the closed loops remain stable in the absence of rapid rotation. In a rapidly rotating centrally concentrated potential, the closed loop (or $x_{1}$ ) orbits are also destabilised - and general loop orbits can be chaotic even though they do not pass close to the centre.

While the horizontal stability of periodic orbits depends only on the potential axis ratio in the plane, their vertical stability crucially depend on the flatness of the halo. In general, more and more orbits become unstable with increasing flatness. For example, it can be seen from Fig. 7 that the fraction of unstable orbits in the oblate models is significantly larger than the corresponding prolate ones with the same axis ratio in the plane. It can be also be seen that the oblate models with $b / a=0.8$ also usually have a larger fraction of unstable orbits than the prolate models with $b / a=0.7$.

The fact that the flatness of the halo is such an important factor in determining the stability properties of the periodic orbits suggests that the existence of disks in the centre of dark halos may also have a similar effect. This was indeed found to be the case and was more pronounced in Model 3 which contains a flatter disk. In the absence of a disk, the halo corresponding to that 

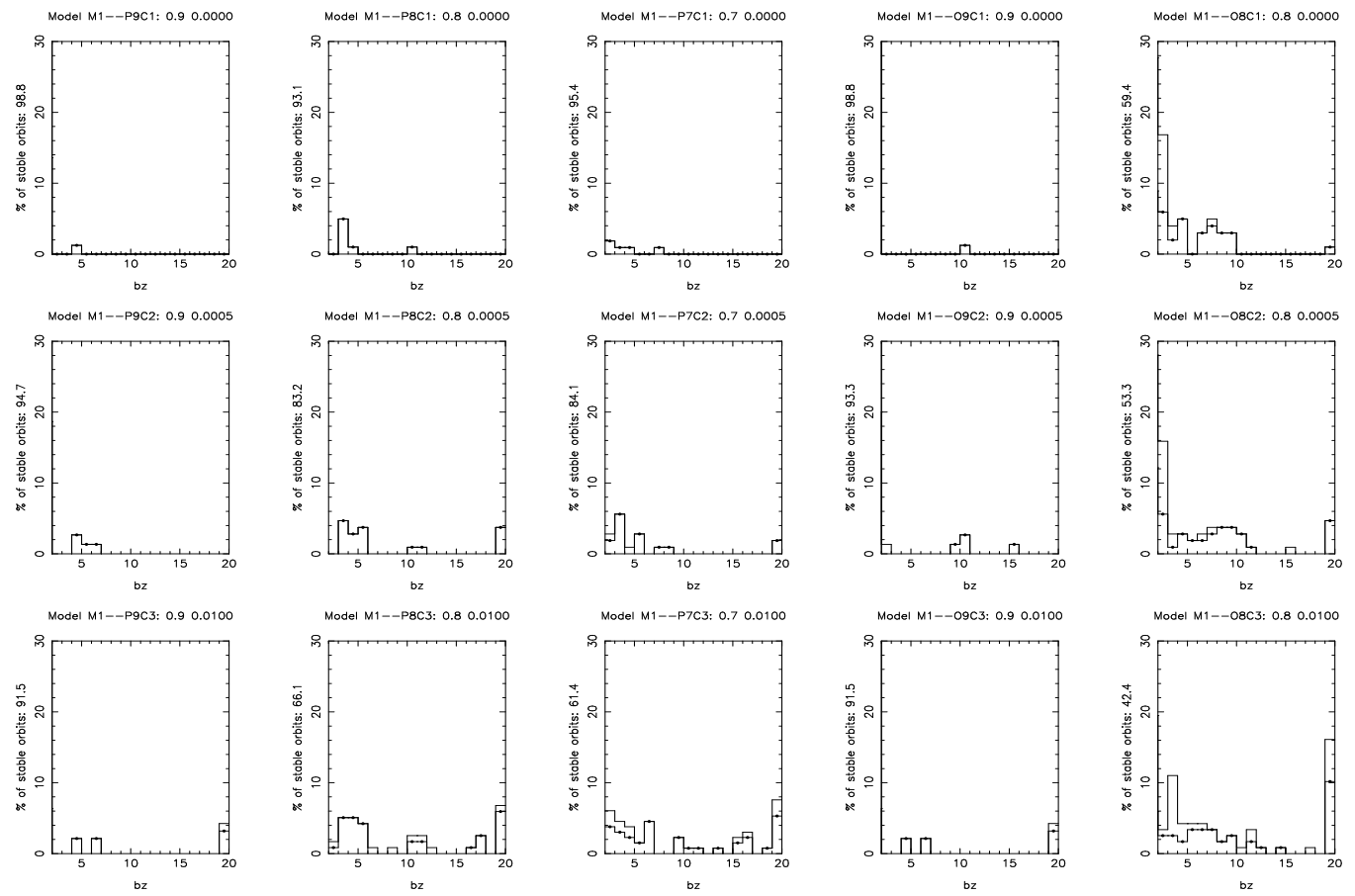

Fig. 7. Distribution of vertical stability parameter $b_{z}$ for the periodic orbits of Model 1 of winding number up to 4 . The different runs are labeled according to the convention described in Section 2.5. Orbits with $\left\|b_{z}\right\| \leq 2.00005$ are labelled stable and the fraction of such orbits is indicated on each diagram

Table 2

\begin{tabular}{c|c|c|c|c|c|c|c}
\hline$G M_{C}$ & $1: 1$ & $1: 2$ & $2: 2$ & $2: 3$ & $3: 3$ & $3: 4$ & $4: 4$ \\
\hline 0.0000 & $0 / 8$ & $3 / 6$ & $19 / 31$ & $21 / 23$ & $3 / 11$ & $6 / 18$ & $1 / 4$ \\
\hline 0.0005 & $0 / 8$ & $10 / 13$ & $21 / 31$ & $22 / 28$ & $5 / 13$ & $2 / 10$ & $4 / 4$ \\
\hline 0.0100 & $0 / 8$ & $14 / 18$ & $29 / 40$ & $18 / 23$ & $9 / 13$ & $3 / 8$ & $6 / 8$
\end{tabular}

Fraction of unstable orbits of different multiplicities in Model 1 with oblate halo and $b / a=0.8$ AND GMC=???. PLUS THE 1:1 ARE 2:2 ARE ETC!!

model has a significantly smaller fraction of $z$ unstable orbits than when the disk is added (Fig. 8). This is in contrast to the case of horizontal stability where actually the reverse is true (since the presence of an axisymmetric disk decreases the asymmetry of the potential in the disk plane).

Between horizontal and vertical instability one usually finds a large fraction of unstable periodic orbits. The persistence of significant numbers of stable low order orbits however means that one has a "mixed" phase space. This is true of all orbit families as can be seen in Table 2. Results for other models are 

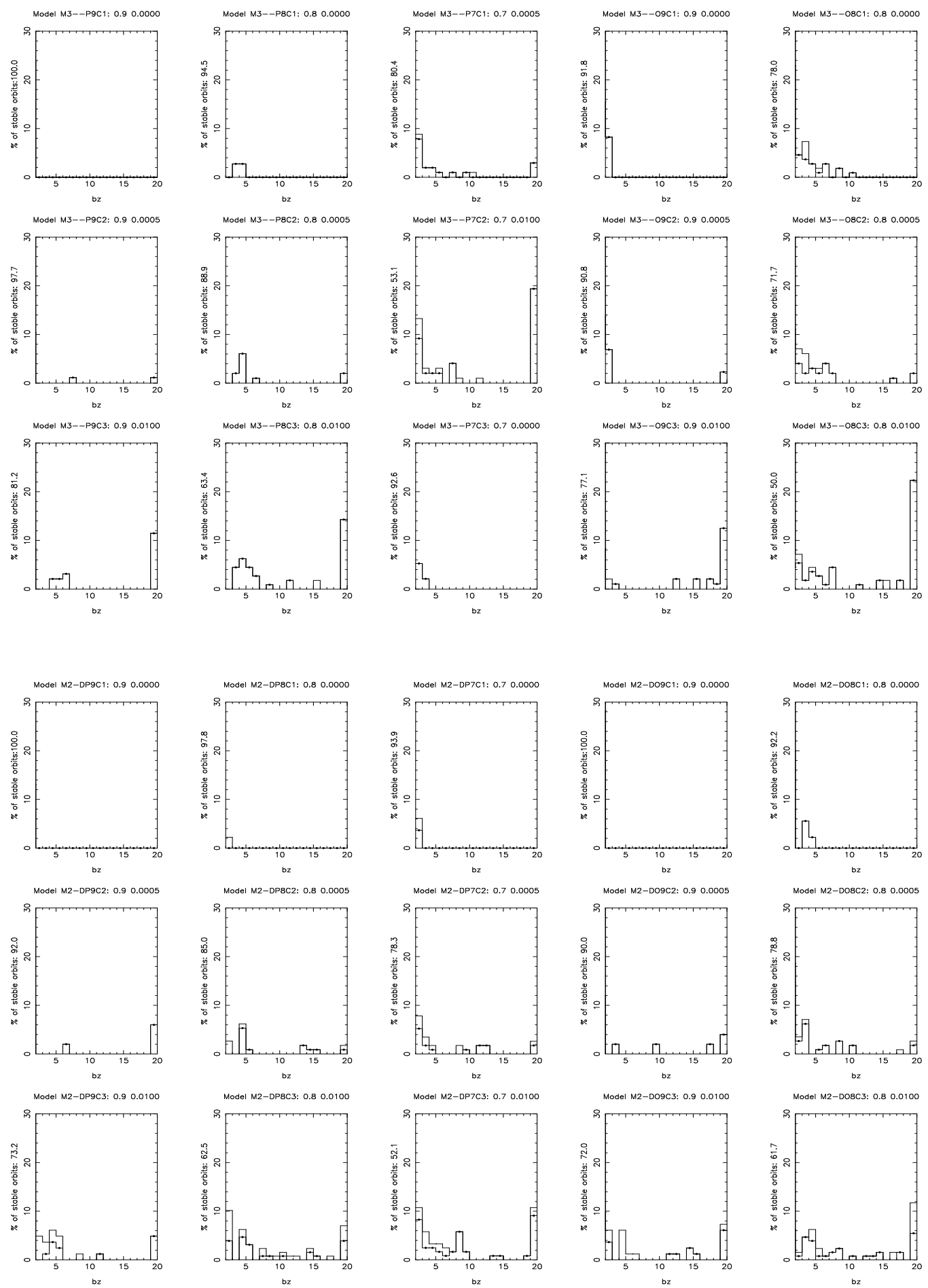

Fig. 8. Distribution of the vertical stability parameter for Model 3 (top) and Model 2 (bottom) but with $G M_{D}=0$ (which is therefore roughly equivalent to Model 3 without a disk). Notation is the same as in Fig. 7 
qualitatively similar. There are important differences between the response of such systems to additional external perturbations, and that of integrable or uniformly hyperbolic systems which are characterised by either KAM or structural stability respectively (see Section 4.2 for more detail).

\section{Stability properties of ensembles of general orbits}

\subsection{Models with disk, halo and central mass}

\subsubsection{Method}

We have looked at the stability of the periodic orbits which parent (or deflect) the general ones. We now try to get an idea of the global structure of the phase space by studying the stability of general orbits.

In each model, we have integrated an ensemble of nearly a hundred trajectories and calculated their maximal Liapunov exponents. The set of 12 first order differential equations - representing the six differential equations of motion and their associated variational (linearised) equations - were numerically integrated using the variable order, variable step size, Adams method as implemented in the NAG library routine D02CJF with a local tolerance of $10^{-14}$ per time step. The relative energy change along a trajectory was smaller than $10^{-10}$.

A two parameter "startspace" (Schwarszchild 1993) of initial conditions was chosen as follows

$$
\begin{aligned}
x_{i j} & =i \times 2 \\
y_{i j} & =0.0 \\
z_{i j} & =0.02 \\
\dot{x}_{i j} & =0.0 \\
\dot{y}_{i j} & =0.02 \times j-0.02 \\
\dot{z}_{i j} & =0.0,
\end{aligned}
$$

where the integer $i$ was varied from $i=1$ to $i=8$, while $j$ took values ranging from $j=1$ to $j=12$. In total, therefore, we have 96 orbits started from near the disk plane. The small initial $z$ value is included to test the vertical stability of the orbits, which in most cases is a measure of the degree of stochasticity of a given orbit. This choice of initial conditions starting near

the disk plane is motivated by the aims outlined at the end of Section 1 . The 
choice of range and resolution in the coordinate $x$ will mean that for Model 1 and 3 one will be studying areas outside the core (up to eight core radii), so that the stability results will mainly concern halo orbits and eccentric high energy stellar trajectories. In Model 2 one will be concerned with orbits inside the core and within two or three core radii. A large fraction of these can be interpreted as stellar orbits in the dynamically hot inner areas where, in the absence of a central mass contribution, motion is likely to be on box-like orbits characteristic of the harmonic core. (The extent of the effective - disk + halo - cores of our models can be inferred from the rotation curves where they are represented by the rising part of these curves).

The fact that the value of $\dot{x}_{i j}$ is always zero means that we are considering only orbits whose trajectories are normal to the $x-z$ plane at least once during their crossings of that plane near $z=0$ (choosing the initial conditions on the $x-z$ plane is no restriction, since, by symmetry, all orbits must cross it at least once). This condition is satisfied by both box and loop orbits - because the former can be regarded as independent 1-dimensional oscillations about the axial orbits while the latter are parented by the closed loops, which are themselves symmetric about the $x$-axis. One can also expect many highly chaotic orbits to satisfy this condition during their near random time evolution. The choice of a two parameter space of initial conditions instead of a three dimensional one also facilitates the representation of the results. Although there is no guarantee that all type of orbits will be caught in this space (for example orbits parented by the $x-y$ banana family which is asymmetric with respect to the $\mathrm{x}$-axis might not be caught), a representative sample should. In addition, this procedure avoids the non-uniqueness arising when the full 3-parameter space is used (where essentially the same trajectory may be integrated more than once: Schwarschild 1993).

Our startspace covers a large range of energies. However the variation of energy with the chosen initial conditions is slow enough so that orbits can be grouped together into a few small-energy intervals (as was done in Fig. 4 and Fig. 5). In Fig. 9 we have numbered our orbits sequentially and plotted the orbit number $N_{i j}=12(i-1)+j$ against the intersection of the zero velocity surface with the $x-y$ plane (assuming a spherical halo). Only a few orbits are confined within the same zero velocity circle, but the variation of the radius of this circle with the orbit numbers is seen to be slow enough so that all energy ranges are sampled reasonably well.

For each trajectory, we calculate the numerical maximal Liapunov exponent and record its value at $50000 \mathrm{Myr}$. This choice, which corresponds to a few Hubble times, ensured proper convergence for most orbits and facilitates the extraction of information from distribution of exponents (i.e., classifying trajectories according to their stability properties). We will be comparing the stability properties inferred from calculating the Liapunov exponents over such 

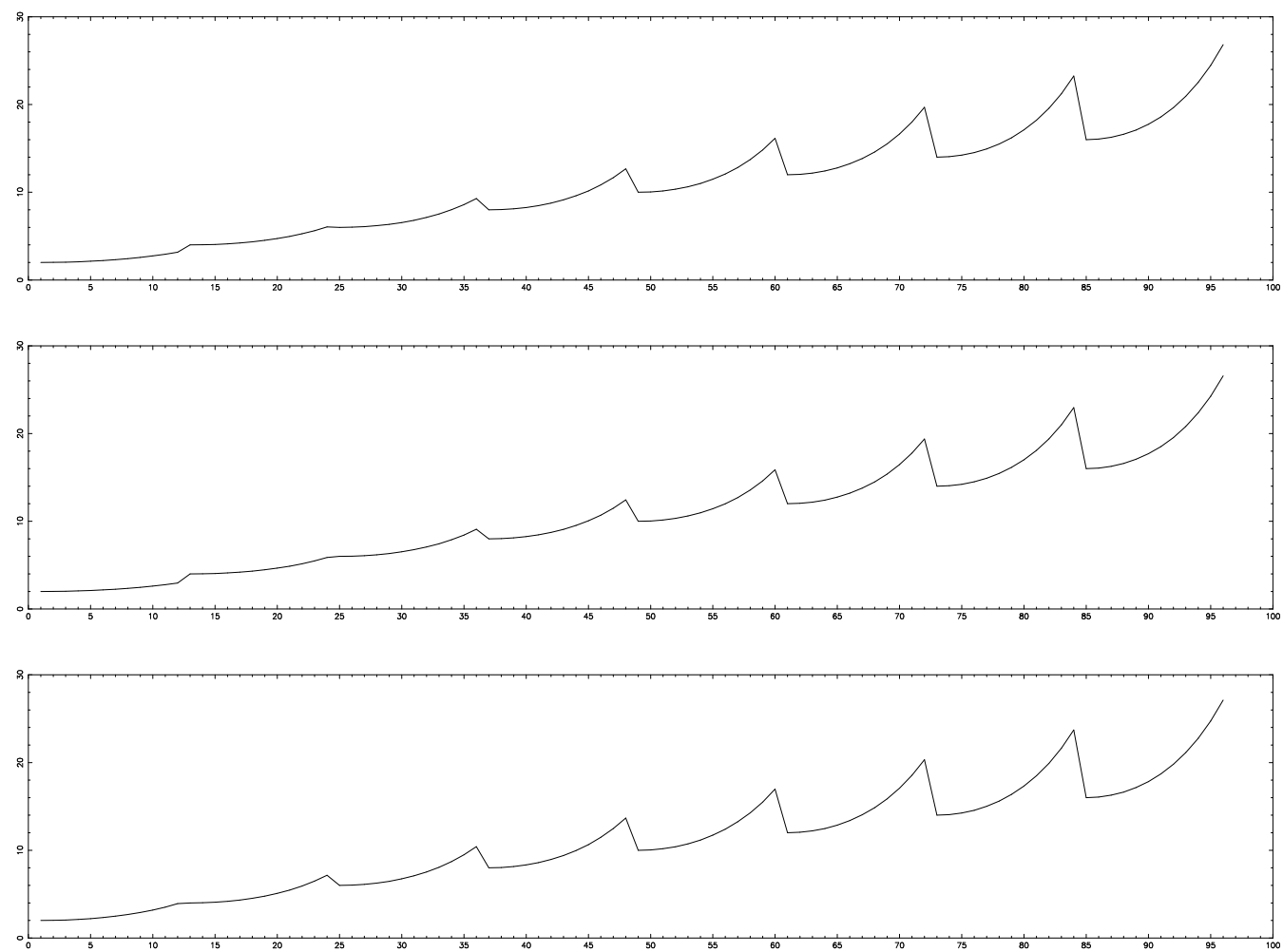

Fig. 9. Intersections of the zero velocity surfaces confining the orbits with initial conditions given by Eq. (12) to (16) with the $x-y$. The orbits are numbered according to the rule $N_{i j}=12(i-1)+j$. Top: orbits of model 1. Centre: orbits of Model 2. Bottom: orbits of Model 3

time-scales with the actual $z$ stability of trajectories over smaller times-scales (comparable to a Hubble time).

A well known difficulty (e.g., Udry \& Pfenniger 1988; Merritt \& Fridman 1996) is the absence of a universal criterion by which to distinguish if a given numerical Liapunov exponent describes a chaotic orbit or a regular one. We use the following estimate for the cutoff value of the exponent (below which we call an orbit regular). Consider a circular orbit of radius $R$ in the plane of an axisymmetric mass distribution. In the outer regions the rotation velocity is constant and $\sim v_{0}$, so that the differences between the angular frequencies of this orbit and another at a radius $R+\Delta R$ is, in the linear approximation, given by

$$
\Delta \omega=\frac{v_{0}}{R^{2}} \Delta R
$$

The phase distance between these orbits after a time $\Delta t$ is therefore

$$
D=R \Delta \omega \Delta R \Delta t=\frac{v_{0}}{R} \Delta R \Delta t
$$


Differences in all other phase space coordinates of these two orbits are constant, so that their contribution to the time dependent maximal Liapunov exponent is negligible by comparison. This quantity is then given by

$$
\lambda(t)=\frac{1}{t} \log \left(\frac{\left\|\frac{v_{0}}{R} \Delta R t\right\|}{\|\sqrt{6} \Delta R\|}\right)
$$

which for Model 1 at $10 \mathrm{kpc}$ for example has a value of $\sim 1.2 \times 10^{-4}$ at 50 $000 \mathrm{Myr}$, which implies an exponentiation time-scale of the order of a Hubble time. In practice, it is found that the vast majority of regular orbits (those for which the Liapunov exponent decreases monotonically as $\ln t / t$ even when integrated for very large times) have time dependent Liapunov exponents of the order of $\sim 5 \times 10^{-5}$ at $50000 \mathrm{Myr}$ (i.e., with an exponentiation time scale longer than most estimates of the Hubble time). We will therefore consider orbits with a maximal exponent less than $10^{-4}$ to be regular. The maximal exponent was calculated by the "standard algorithm" of Benettin et al. (1976) with a renormalisation interval of 150 Myr.

\subsubsection{Results}

We will illustrate our results using greyshade diagrams of our startspace. In these diagrams the (white) background will correspond to orbits with Liapunov exponent less than or equal to $10^{-4}$, while the (black) foreground will correspond to slots on the startspace occupied by the initial conditions of orbits having Liapunov exponents of $4 \times 10^{-3}$ or greater. This value implies an exponentiation time-scale smaller than a rotation period at $10 \mathrm{kpc}$ in the plane of the axisymmetric Model 1. Ensembles of trajectories with such large exponents are known to relax to time invariant distributions filling all their available energy subspace in just a few exponentiation times (e.g., Merritt 1996). Indeed it was found here that with almost all such trajectories for which this property (ergodicity) was checked (a total of about 50 orbits), an invariant distribution was reached in less than a Hubble time.

Fig. 10 shows the Liapunov exponent greyshade diagrams for the orbits of Model 1 for different values of the axis ratio, triaxiality parameter and central mass which we have considered (Section 2). As can be seen, the results are what is to be expected from both the general discussion of Section 3.1 and the analysis of periodic orbits of Section 3.2 In the absence of a central mass (top row) and when the halo is spherical (far left) all orbits are regular. As one moves towards a more asymmetric halo however (prolate model with $b / a=0.9$; second from left), some orbits starting with large initial $x$ coordinates start becoming chaotic. These are part of the stochastic layer around the 1:2 vertical resonance which was brought inwards with increasing asymmetry in the halo mass distribution (as is also clear from the subsequent diagrams on the right). 


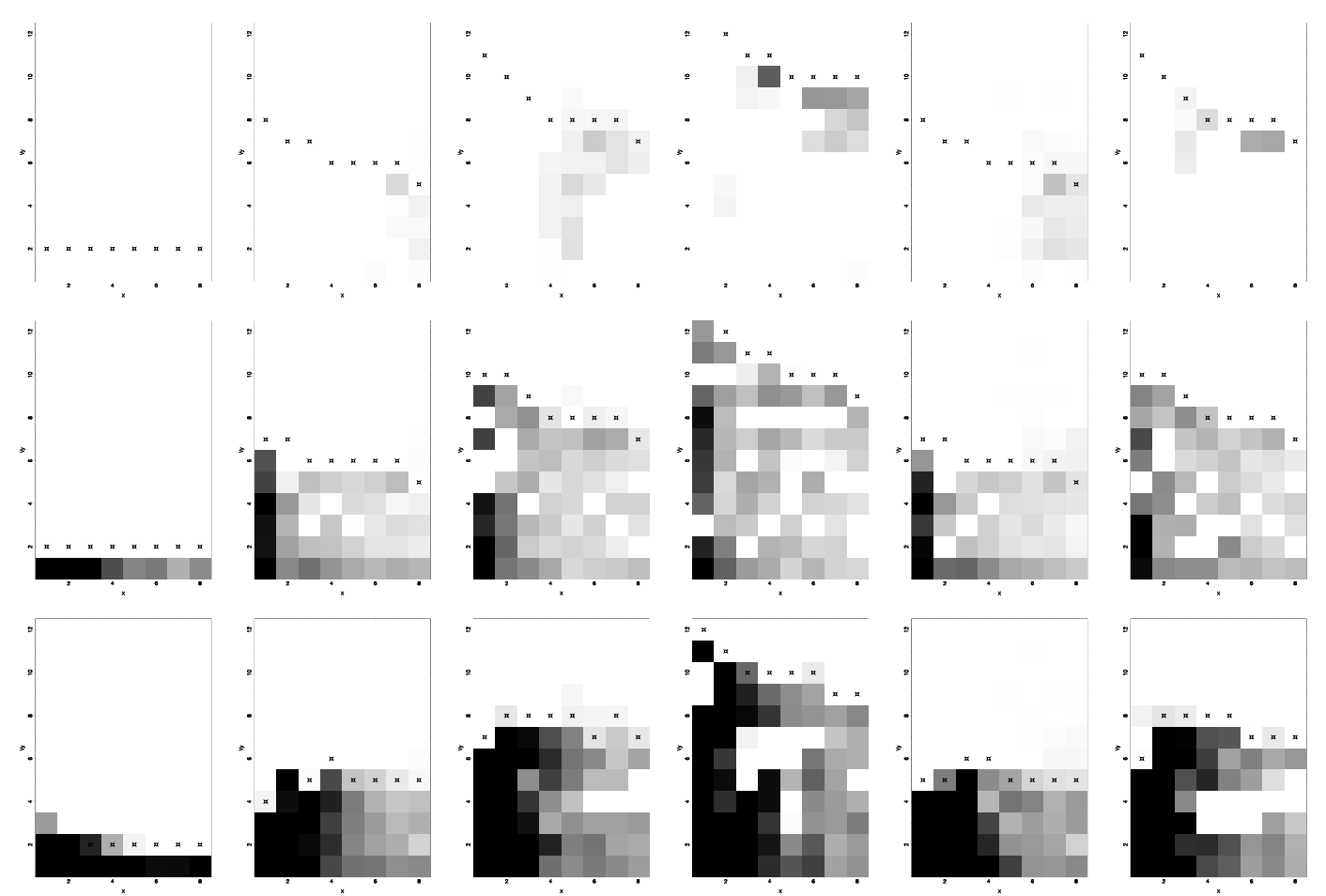

Fig. 10. Greyshade diagram showing the distribution of the maximal Liapunov exponents. The diagrams corresponding to the different runs are the same as in Fig. 6 except that in the first column in the left the diagrams correspond to the case when the halo is spherical which was not considered in the aforementioned figure. The initial conditions are obtained according to the prescription in Eq. (12) to Eq. (16)

In the case $b / a=0.7$ the asymmetry in the plane is large and the horizontal $3: 4$ resonance is broad enough to give rise to a significant stochastic layer around unstable antipretzel orbits, where "trapped" chaotic motion occurs.

The greyshade diagrams of Liapunov exponents for runs with $G M_{C}=0.0005$ are shown in the second row of Fig. 10. As we have seen (Section 3.1), in the two dimensional case, this central mass is enough for destabilising the $x$-axis orbit and to cause most trajectories in the central regions to become chaotic. Here we also see that remarkably modest deviations from axisymmetry (the models with 0.9 halo potential axis ratios in the plane are compatible with observations of present day galaxies) can have a large effect on the orbital structure when a modest central mass is present - although, as expected, the more asymmetric systems contain a larger fraction of initial conditions from which chaotic trajectories may start. It is interesting to note here that chaotic behaviour is not limited to the central box orbits only, but is significant even at $16 \mathrm{kpc}$ from the centre - contrary from what may be expected from simple arguments (e.g., Gerhard \& Binney 1985). However, the existence in the region once occupied by the box orbits, of a large number of trajectories which are either regular or have small Liapunov exponents, will mean that the 
halo would not rapidly (i.e., over a dynamical time or so) lose triaxiality.

The addition of a larger central mass (bottom row) increases both the exponentiation rates of individual chaotic orbits and the fraction of such orbits. A large fraction of the chaotic orbits now have exponentiation time-scales of the order of a dynamical time and, therefore, evolution is likely to take place on a time-interval of that order. This is compatible with the results of Merritt \& Quinlan (1997) where it was found that triaxial figures (representing elliptical galaxies in their case) do indeed lose their asymmetry on a time-scale of a few dynamical times when the central mass is a few percent of the total mass of the galaxy (which is the case here for the inner 10-20 kpc). Nevertheless, as we have seen in Section 3.1, some higher order closed boxlet orbits will remain stable even for such large central masses and asymmetries. The white "islands" seen in the diagrams here correspond to quasiperiodic orbits parented by these. In addition, there are, in general, fewer chaotic orbits in the central areas because many are parented by closed loops created by the central mass (because of the destruction of the harmonic core and the more axisymmetric potential). Due to the reasons mentioned in Section 3.1, none of these can be chaotic.

We have also calculated the Liapunov exponents for trajectories starting from the same initial conditions with the exception that $z=0$. The main difference found was that, in the two dimensional case, significantly fewer orbits are chaotic, but those that are, usually have larger Liapunov exponents than the corresponding ones in the three dimensional case. The fact that chaotic behavior is more widespread in the three dimensional model is probably due to the role of the role played by $z$ instabilities in the closed periodic orbit families (Section 3.1) - that is, some of the closed orbits that parented the regular islands in the two dimensional case are $z$ unstable and therefore cease to parent these orbits when a $z$ perturbation is applied.

We have recorded the maximum value of $\|z\|$ along the trajectories of the orbits for $t \leq 20000 \mathrm{Myr}$, with the $z$ coordinate being sampled at every renormalisation interval (sampling only until $t=20000$ means we catch orbits with effective $z$ instability over a period comparable to a Hubble time). These values have also been arranged in greyshade diagrams. The background value for these diagrams was taken to be equal to $0.1 \mathrm{kpc}$. Clearly, trajectories with larger maximum $z$ excursions are unlikely to be undergoing stable independent $z$ oscillations (as would happen if the vertical motion was decoupled). The foreground was chosen as $5 \mathrm{kpc}$. The results for Model 1 are shown in Fig. 11. As is clear from this figure, the $z$ stability of orbits is usually correlated with the value of their Liapunov exponents (Fig. 10). There are however orbits that have fairly large Liapunov exponents but are confined to the disk plane (for example those of Model M1-P7C3 at $i=7,8$ and $j=7$ ). These orbits start near regular two dimensional boxlet orbits and remain trapped 


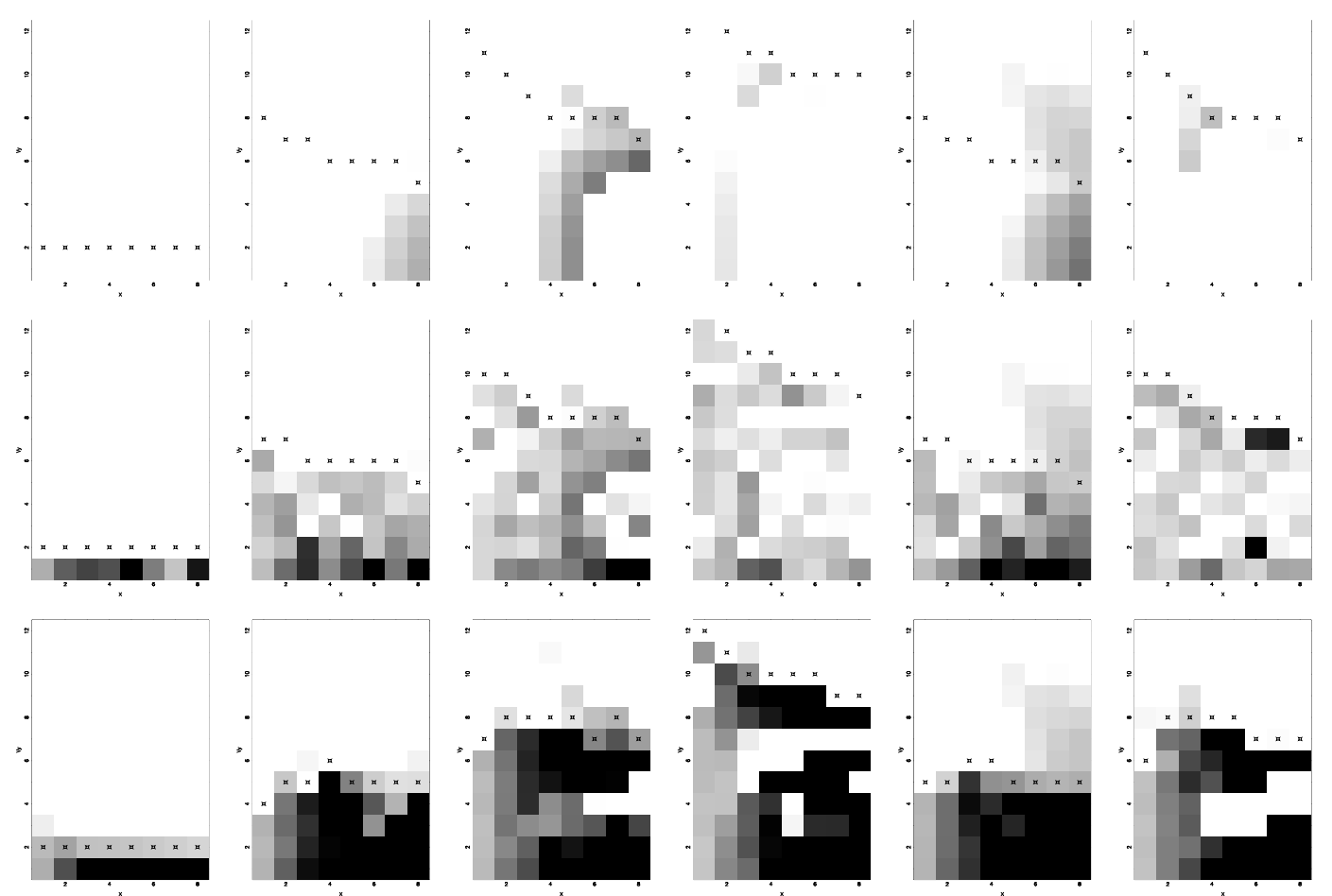

Fig. 11. Greyshade diagrams illustrating the vertical stability of orbits starting near the disk plane. The positions of the diagrams corresponding to runs with different system parameters $G M_{C}$, T and $b / a$ are the same as in Fig. 10

near the latter for very long times. There are also some orbits that have fairly large $z$ excursions but small Liapunov exponents. These are mainly trapped around the vertical 1:2 resonance.

The vertical 1:2 resonance is also present in flat axisymmetric potentials where it is also a source of $z$ instability for trajectories starting near the disk plane. This resonance is too far out along the $x$ axis to be of interest for models with large core radii but is moved inwards when the core radius is decreased. Below $R_{0}=2$, its position along the long axis does not vary much with core radius or the existence of a central mass, but depends primarilly on the flattening of the density distribution. Fig. 12 shows the maximum $z$ excursions for ensembles of trajectories in models where $R_{0}^{2}=0.5$. As can be seen, while the stability of trajectories passing sufficiently near the centre are affected by the central mass, the main instability region around the 1:2 resonance remains unchanged. This is due to the fact that the central mass, by creating a strong nonlinearity in the potential of the central region, gives rise to a set of high order vertical resonances with connected instability gaps along the axial orbit. In the triaxial case, this causes widespread chaotic behaviour in trajectories that oscillate about the axial orbit - the box orbits. The higher order resonances are not broad however, and therefore have little effect away from the axial orbit itself. Therefore, in the axisymmetric case, all but the lowest angular momentum orbits remain unaffected by the addition of a central mass. 


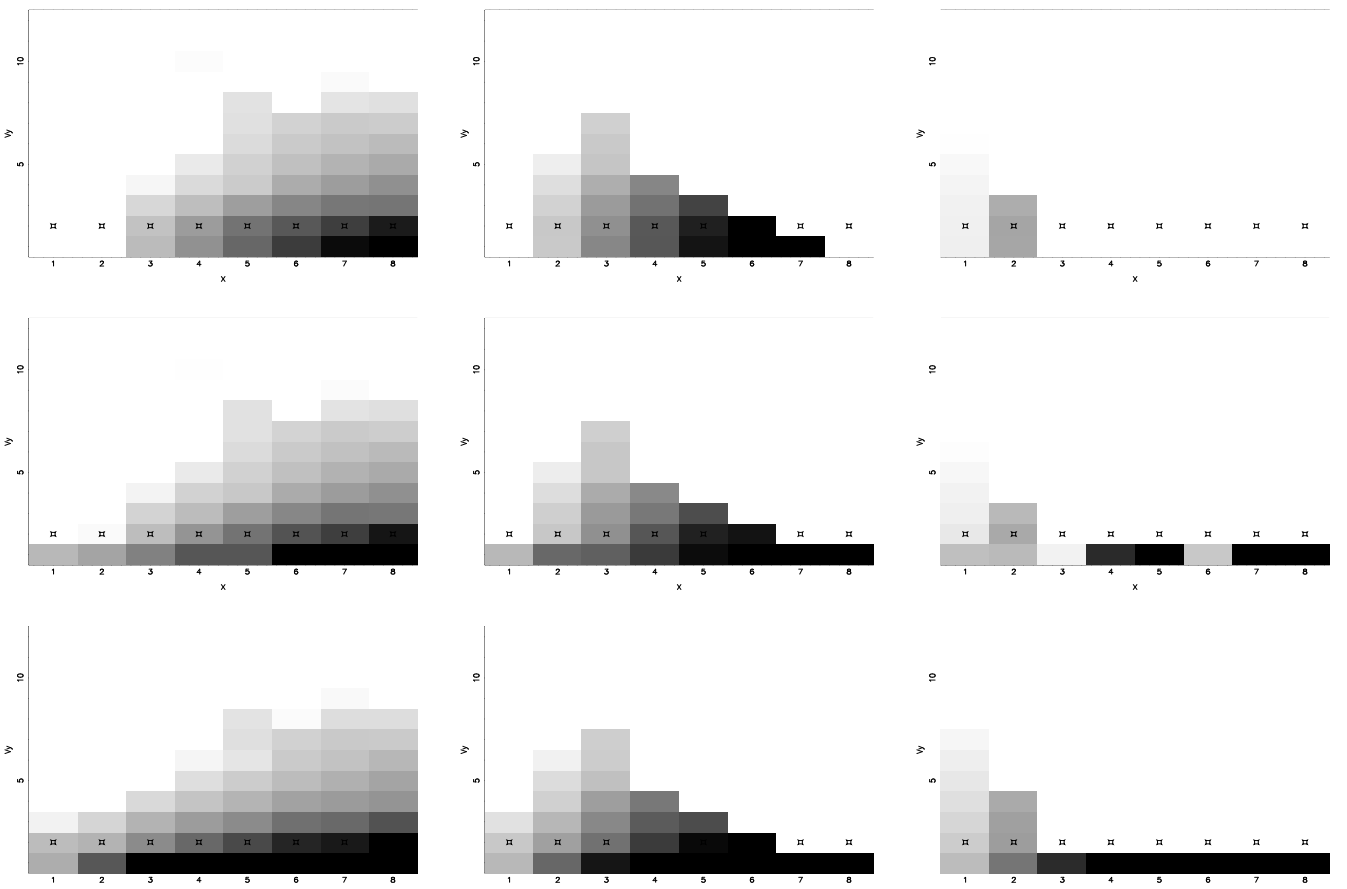

Fig. 12. Greyshade diagrams illustrating the vertical stability of orbits starting near the disk plane of axisymmetric models with $R_{0}^{2}=0.5$ with (from left to right) $c / a=0.9, c / a=0.8$ and $c / a=0.7$ and (from top to bottom) $G M_{C}=0$, $G M_{C}=0.0005, G M_{C}=0.01$.

Coming back to the triaxial case now, we examine in more detail the relation between the maximum of the absolute value of the $z$ coordinates of our trajectories, and the corresponding Liapunov exponents. This is shown in Fig. 13. Here we have plotted the values of the maximal Liapunov exponent against the ratio of the largest $z$ excursions of the trajectories of Model 1 to maximum value of the $z$ coordinate on the zero velocity surfaces of the orbits. (i.e. the $z$ coordinate of the zero velocity surface at $(x, y)=(0,0)$, which is the maximum possible $z$ value a trajectory can reach). It is clear that a strong correlation exists between the two quantities. For small values of the Liapunov exponent the relationship appears to be linear, while for larger values most orbits reach the maximum $z$ value possible. This correlation is remarkable given the fact that the sampling of the $z$ coordinates is done only up to $t \leq 20000$ while the exponent is calculated until $t=50000$. While some of the scatter is due to "trapped" chaotic orbits, only in a few cases did these have large Liapunov exponents. Thus there appears to be a strong correlation between the ergodic properties of the trajectories over a Hubble time and the Liapunov exponents calculated over significantly longer times. And indeed it was verified that trajectories with the larger Liapunov exponents showed significant $z$ excursions within a few tens or hundreds (depending on their energy) Myr, while for trajectories with small Liapunov exponents a significant fraction of the 20000 Myr period was needed before the $z$ instability manifested itself (if at all). We 

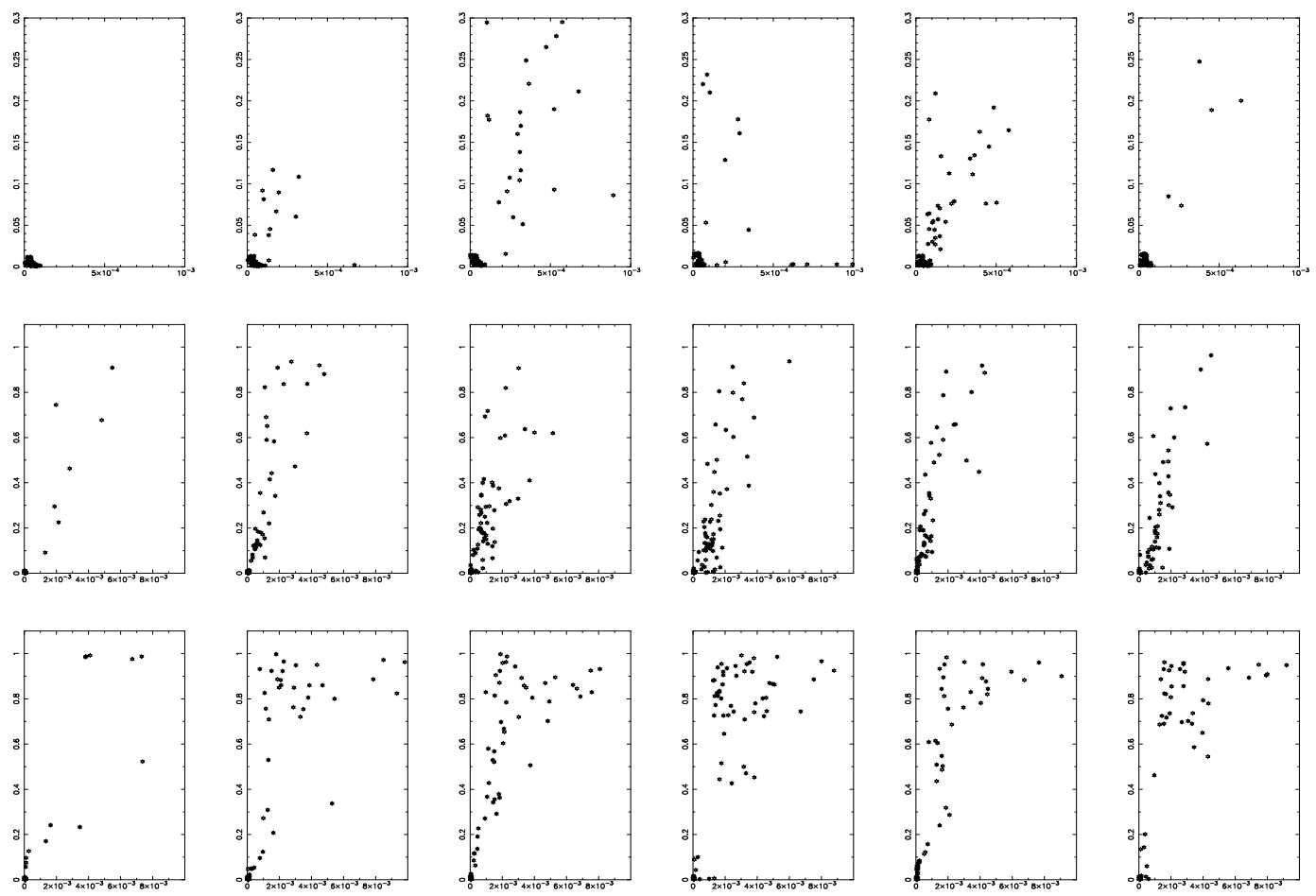

Fig. 13. Correlation between the values of the Liapunov exponents of trajectories of Model 1 (plotted in the abscissa) and the ratio of the largest $z$ excursions to the maximum possible $z$ excursions determined by the largest value of the $z$ coordinate on their zero velocity surfaces. The different diagrams represent runs with the same system parameters as the corresponding diagrams in Fig. 10

will see below however that the correlation between $z$ excursion and maximal Liapunov exponent is much weaker in the case when rapidly rotating bars are present.

The characteristic features distinguishing Models 2 and 3 from Model 1 are the large halo core radii (6 kpc compared to $2 \mathrm{kpc}$ in Model 1) and the more dominant disk. In Model 2 the disk has a larger total mass while in Model 3 it is more centrally concentrated. This amounts to decreasing the nonlinearity (especially in Model 2) of the potential and decreasing the asymmetry in the central regions (especially in Model 3).

In Model 2, the above effects will mean that the main resonances (both vertical and horizontal) are moved outwards. It follows that one will not be observing the $z$ instability around the 1:2 resonance which has moved beyond our range of initial conditions. This can be clearly seen from Fig. 14, where the Liapunov exponents greyshade diagrams for Model 2 are shown. In Model 3, there is some instability around the 1:2 resonance but it is limited and almost all orbits near the plane are again stable. As usual, this situation changes when a central mass is added. Even the mildly asymmetric cases with $b / a=0.9$ contain significant fractions of chaotic trajectories - even when the central 


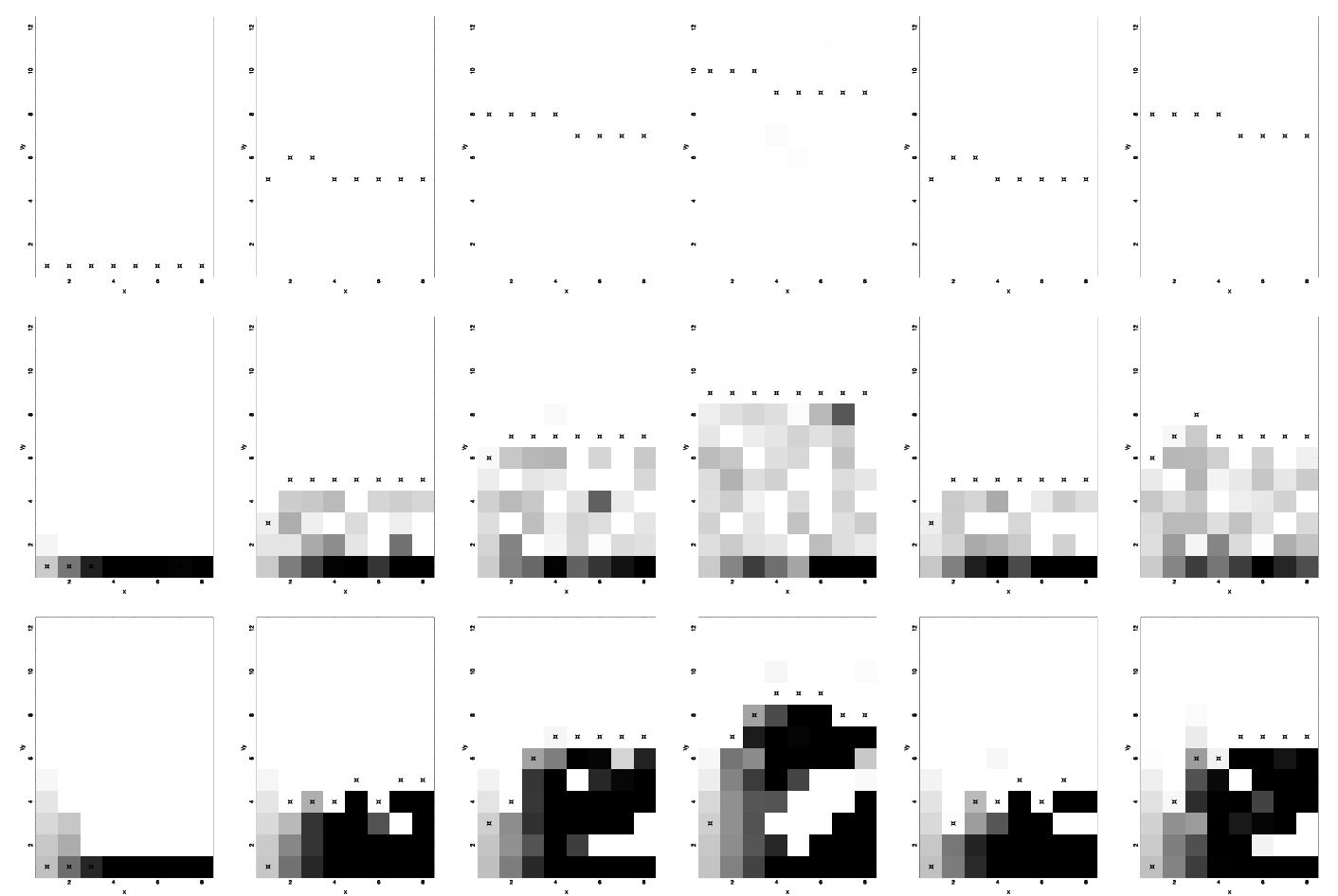

Fig. 14. Distribution of Liapunov exponents for orbits of Model 2. The different diagrams correspond to runs with the same system parameters as in Fig. 10

mass is small. So, as in Model 1, most box orbits are again replaced by chaotic ones. One important difference with Model 1 however is that, in runs with a large central mass, most orbits in the inner areas which have definite sense of rotation are not parented by the 1:1 loops. Many of these are chaotic; they appear to be repelled by unstable looplet orbits (cf. Section 3.2). There is also an important difference in interpretation: while in Model 1 we were concerned mainly with trajectories which venture way beyond the small harmonic core - and are therefore either halo trajectories or those of stars born (or heated) into eccentric trajectories - in Model 2, many trajectories we calculate are completely confined within the harmonic core and therefore must correspond to stellar trajectories born on box orbits (because of the absence of loop orbits completely confined within the core). These are subsequently destabilised by the accretion of central mass (cf. Section 4.4.1) and are therefore candidates for forming extensive bulge-like structures out of disk material.

\subsection{The effect of discreteness noise}

Up to now we have assumed that stars in a galaxy move in the mean field potential produced by the smoothed out mass distribution. This is of course the usual assumption made in galactic dynamics. However, it has been known for some time that, at least in some cases, this picture may not be accurate 
(Pfenniger 1986; Kandrup 1994; Merritt \& Valluri 1996). In considering the effect of discreteness on the dynamics, a first approximation is just to introduce random kicks to the trajectory in the smoothed out potential. We will model these as perturbations to the velocity which are sampled from a Gaussian distribution. This may not be a very accurate representation of discreteness effects in a real $\mathrm{N}$-body system where one can imagine all sorts of resonance effects between the different degrees of freedom (El-Zant 1997), but may be useful as a first approximation.

To examine the effects of discreteness noise, we have perturbed the velocities along the trajectories of Model 1 at intervals of $1 \mathrm{Myr}$. The perturbations to each component of the velocity were sampled from a Gaussian distribution with zero mean and dispersion of $10^{-4} \mathrm{kpc} / \mathrm{Myr}$, produced using the NAG routine G05DDF. Although this procedure does not explicitly conserve energy (the magnitude as well as the direction of the the velocity is changed, unlike the scheme of Goodman \& Schwarschild 1981), because of the smallness of the perturbation however, the energy is conserved to better than $1 \%$ (usually to a few parts in a thousand) over a period of $50000 \mathrm{Myr}$. That means, if one assumes that errors in the energy grow according to a $\sqrt{t}$ diffusion law, the energy relaxation time is $\sim 10^{5}$ Hubble times. The velocity relaxation time resulting from this perturbation is of the order of

$$
t_{r}=\frac{v_{0}^{2}}{3} \times\left(10^{4}\right)^{2} \sim 10^{6} \mathrm{Myr},
$$

which is about a hundred Hubble times.

Fig. 15 shows the greyshade diagrams for the maximum $z$ excursion of these perturbed orbits (which are to be compared with those of Fig. 10). As is apparent from these figures, even such small perturbations as those introduced here can have a non-negligible effect on the orbital structure. For the spherical case, the grey hue is compatible with the $z$ excursion expected from normal diffusion caused by the the perturbation the trajectories are subjected to. As can be seen however, for the triaxial models, rather large excursions can take place. These cannot be attributed to standard diffusion, especially because in the flatter models, the $z$ force is generally stronger and trajectories tend to reside for longer times in the central regions (which means they will require larger perturbations to achieve the same maximum $z$ excursions than their counterparts moving in a spherical halo). Instead, these highly unstable trajectories appear near the vertical resonances (especially the 1:2). Thus they are the result of the broadening of the chaotic regions around these resonances, which is a consequence of the external stochastic noise.

For the models with central mass one sees the above effect greatly enhanced. In particular, many of the isolated blank areas, corresponding to stable islands, 

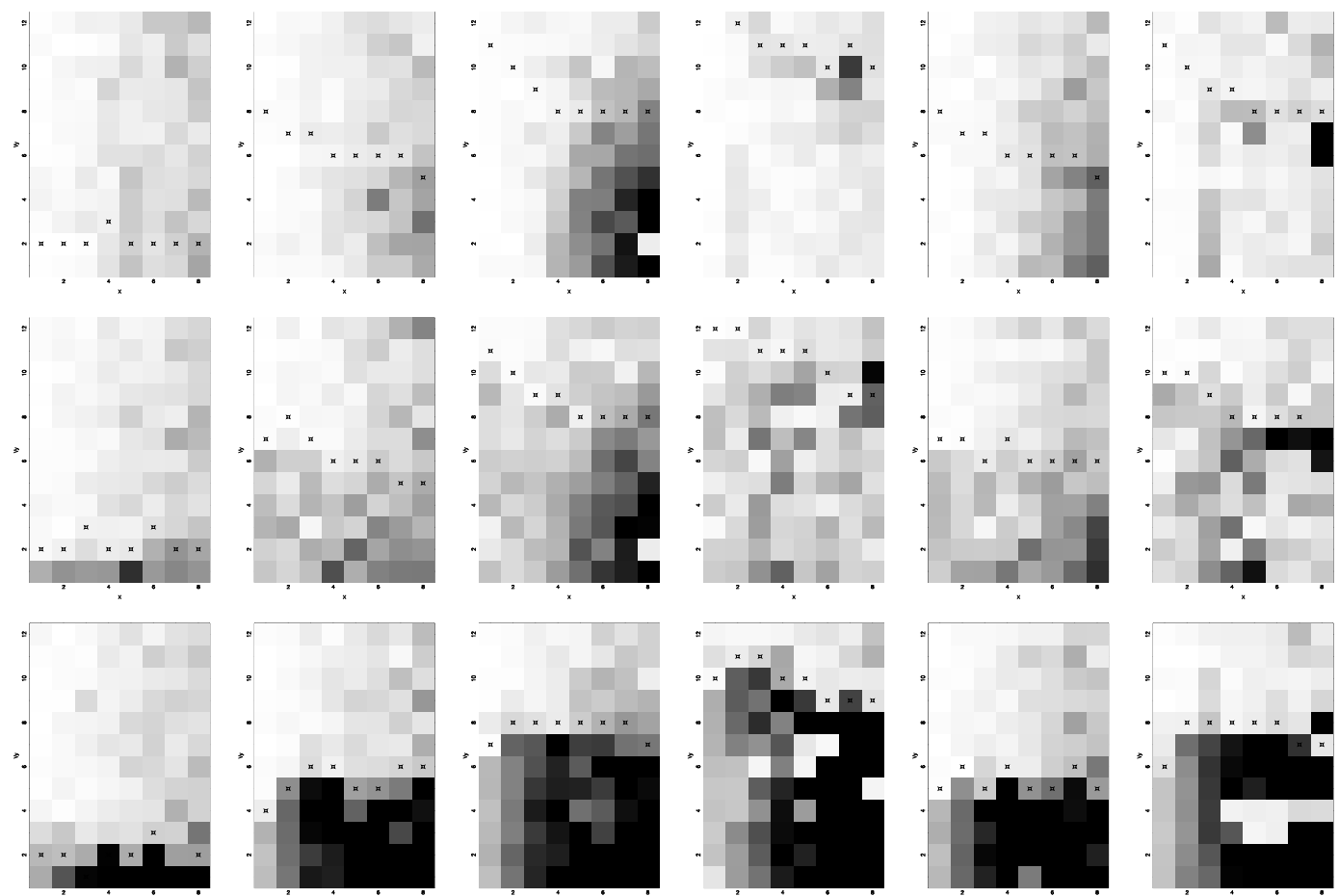

Fig. 15. Greyshade diagrams showing the distribution of the maximum value of the $z$ coordinates of trajectories of Model 1 the velocity components of which are perturbed by noise sampled from a Gaussian distribution with zero mean and dispersion $10^{-5}$. The different diagrams correspond to runs with the same system parameters as in Fig. 10

are now filled with highly unstable orbits. This has the effect that unstable regions merge into connected areas. Also, orbits that were only slightly unstable, may now also have very large $z$ excursions (some venturing out to vertical distances larger than $5 \mathrm{kpc}$ ). On the other hand it is found that, in some cases, orbits that were unstable can be stabilised. This effect however is less common (especially in the case with the models containing stronger central mass). However it was found to be of more relative importance when the dispersion of the perturbation was smaller $\left(10^{-5}\right)$. In that case however the overall effect of the perturbation was much less pronounced.

The question discussed above is that of the stability of qualitative behaviour against perturbations. The KAM theorem (e.g., Arnold 1987) guaranties actual stability against weak non-resonant external perturbations. This means, for example, that stars moving in a smooth spherical potential are not likely to change their qualitative behaviour just because some external Gaussian noise is added. Roughly speaking, such noise will act to move a star from one regular trajectory to another, but the total displacement will amount to the mean strength of the noise multiplied by the square root of the number of kicks a star suffered as a result of the presence of this noise (and will thus have an associated relaxation time given by Eq. 20). This was indeed found 
to be the case for regular trajectories moving near the disk plane of our disktriaxial halo models. On the other hand, very chaotic systems benefit from the structural stability (or "roughness" as it is often called in the Russian mathematical literature) of average behaviour. When the situation is intermediate however, so that neither structural stability nor KAM stability are guaranteed (mixed phase space), non-standard diffusion processes can occur and intermittent behaviour can take place on a variety of scales (Zaslavsky et al 1991; Shlesinger et al. 1993; Klafter et al. 1996). This will mean that the phase space structure is not stable and that weak external noise can have major effects not predicted by standard diffusion processes. It can change the qualitative orbital structure by turning regular or confined orbits into chaotic ones and vice-versa. This state of affairs makes it even harder to justify the assumption that galactic systems are near integrable since, even if the smoothed density distribution produces a potential that does not support too large a fraction of chaotic orbits which visit most of the available energy subspace, many of these could be made to do so by the action of weak noise or other weak perturbations.

\subsection{The effect of rapidly rotating barred perturbations}

To get instability in nearly circular periodic orbits a rotating barred perturbation must be present (Section 3.1). Rapidly rotating bar perturbations are of course present in many (if not most) galaxies and they may play an important role in galaxy evolution (e.g., Sellwood \& Wilkinson 1993). Here we discuss the effect of such a perturbation in a disk galaxy with triaxial halo.

We have now more than one potential source for chaos. First, there are the axial orbit resonances giving rise to chaos in the region occupied mainly by the box orbits (when no central mass is present). Second, there are the circular orbit resonances giving rise to chaos in the $x_{1}$ loops in the rotating barred potential. There is also the central mass which affects the location and width of both types of resonances and invariably increases the fraction of chaotic orbits present in the system. Of course these effects do not simply add up and their interaction may be very complex. The very time dependency of the potential, which means that not even energy is an integral of motion, suggests that this interaction between the various contributions will lead to widespread instability.

Fig. 16 shows greyshade diagrams of the Liapunov exponents of Model 1 with an added additional Ferrers bar with parameter values described in Section 2.6. To accommodate the expected large fraction of chaotic orbits with large initial circular velocities and to concentrate the integration of orbits to the region where the bar plays an important role, we have changed the range of initial 

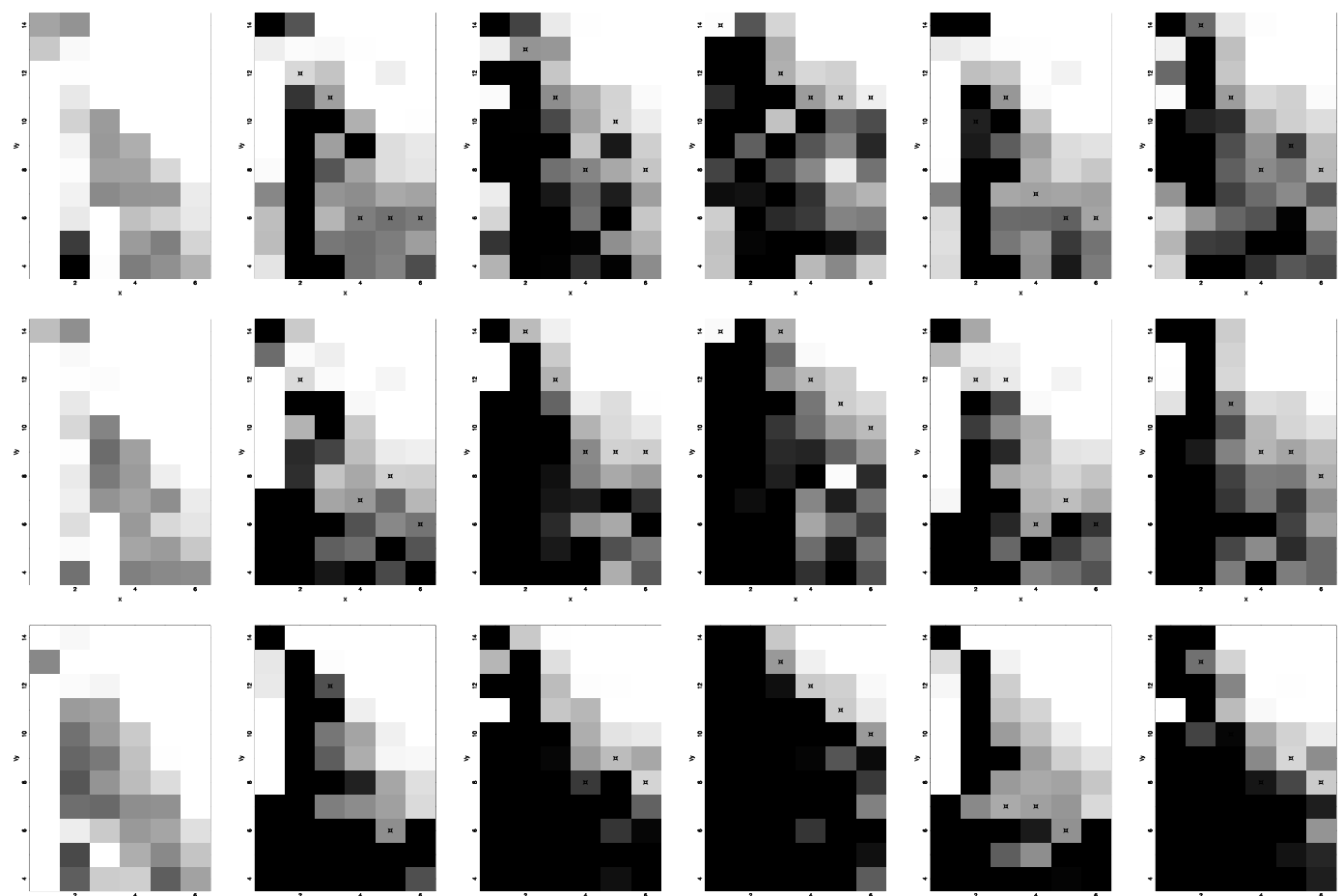

Fig. 16. Greyshade diagram showing the distribution of the Liapunov exponents for orbits of Model 1 when a rapidly rotating Ferrers bar perturbation with parameters described in Section 2.6 is present. The different diagrams correspond to runs with the same halo and central mass parameters as the corresponding diagrams in Fig. 10

condition indices of Equations (12) to (16) to $i=1,6$ and $j=4,14$. Also, due to the more complicated time dependent nature of the potential studied here, it was impossible to integrate trajectories with a tolerance of $10^{-14}$. For most trajectories a tolerance of $10^{-13}$ was adequate, although some required tolerances as high as $10^{-11}$.

Chaotic orbits in the spherical halo potential are mainly those with initial $x$ position starting in the region where the bar dominates and especially in the radii nearing corotation. In this time independent case the above result is standard (because of the accumulation of the resonances near corotation: e.g., Contopoulos 1985). What is somewhat surprising is that the fraction of chaotic orbits and their location does not change much when a central mass is added (even when that central mass is strong). This may be due to the relative weakness of the bar perturbation in our model to those in previous studies (e.g., Hasan et al. 1993) when a central mass was found to considerably alter the phase space structure.

As can be seen however, in the presence of a non-axisymmetric halo, the fraction of chaotic orbits dramatically increases, even when no central mass is present. This effect appears to be directly related to the strength of the time dependent perturbation to the potential (since orbits in prolate potentials with 

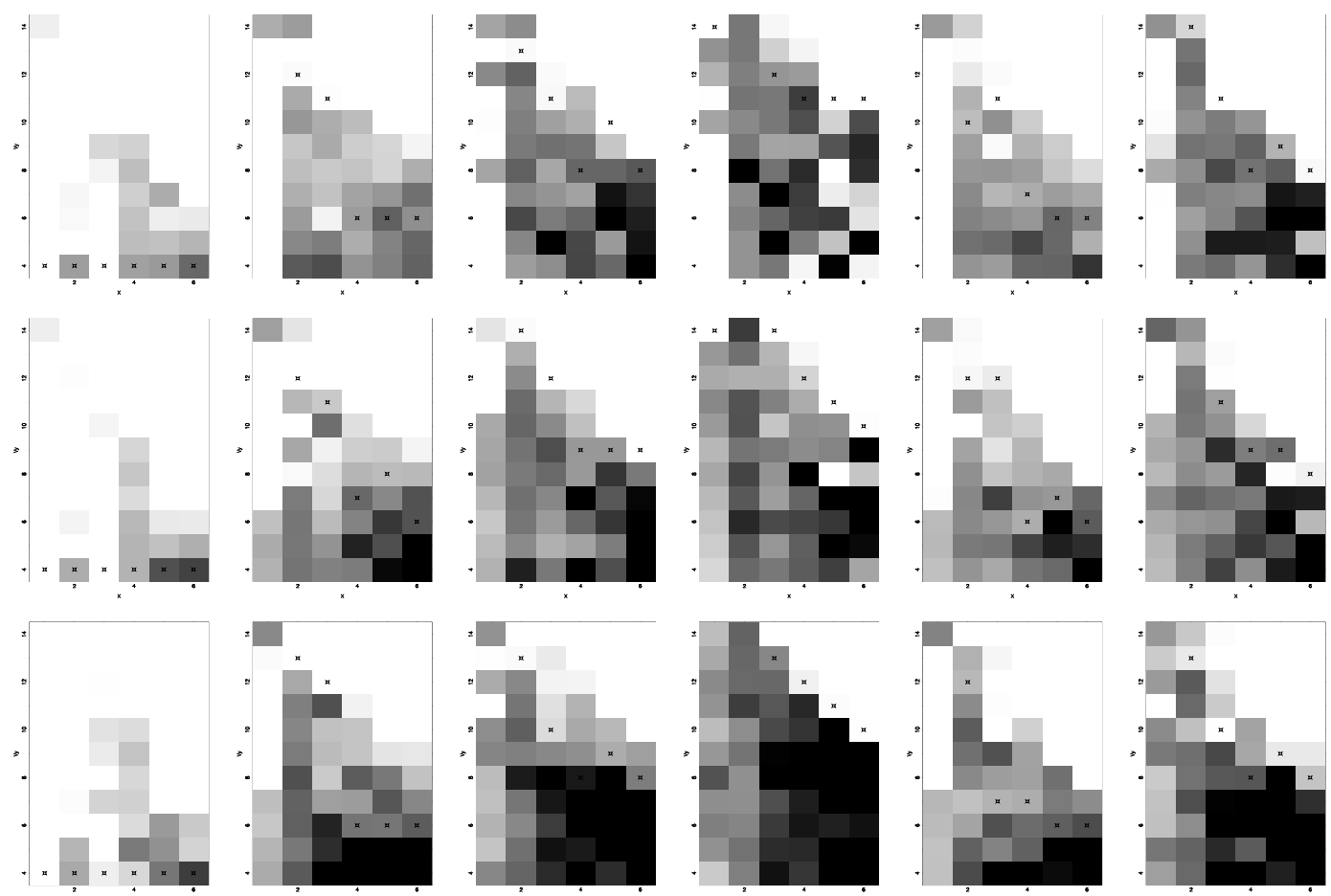

Fig. 17. Greyshade diagram showing the distribution of the maximum $z$ excursions for orbits of Model 1 when a rapidly rotating Ferrers bar perturbation with parameters described in Section 2.6 is present. The different diagrams correspond to runs with the same halo and central mass parameters as the corresponding diagrams in Fig. 10

similar axial ratios $c / a$ to the corresponding oblate ones but with smaller axial ratio $b / a$ in the plane appear to have larger Liapunov exponents). In each diagram, we have also marked with a little square the first of the orbits that has a definite sense of rotation. One can see that, while in the spherical case all orbits have a definite sense of rotation, in the case when the halo is asymmetric, many of the orbits do not have a definite sense of rotation. Although this result can be seen as an obvious corollary of having included the stationary triaxial halo, it can have important consequences. More precisely, it is unlikely that self consistent bars can be built with the help of these orbits, even if the phase space diffusion accompanying the chaotic behaviour does not prevent the building of such structures.

Adding a central mass increases the fraction of chaotic orbits present even further. This is especially true in the central regions where it is most effective, with the larger central mass affecting a wider area. This effect is again most apparent in the more asymmetric models and clearly depends more on the halo potential axis ratio in the plane than normal to it.

If one now looks at the diagrams showing the $z$ stability of the orbits of Model 1 in the presence of the Ferrers bar (Fig. 17), one sees the same general 


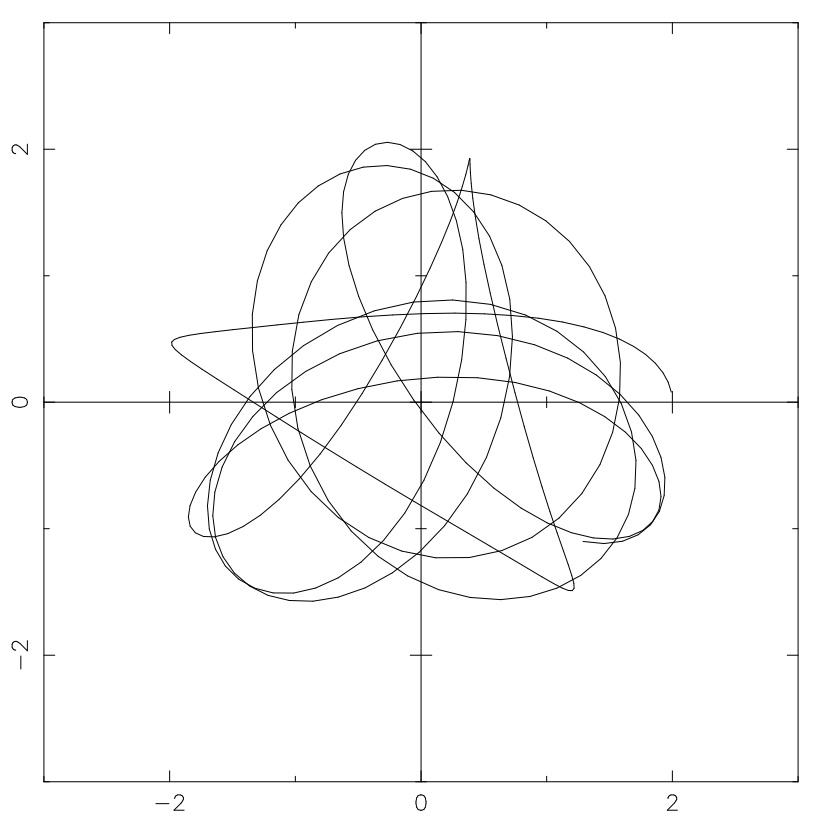

Fig. 18. Highly chaotic trajectory which is trapped in the disk plane. Note the frequent large deflections it suffers at successive crossings

trends as deduced from the Liapunov exponents. An interesting result which can be deduced from these plots however is that many orbits that have a large Liapunov exponent are not $z$ unstable or are very mildly so. This is found to also be mostly true if one samples the $z$ excursion up to the full integration time of 50000 Myr instead of only 20000 as in this figure. This effect is probably due to the fact that the time dependency in the potential is largest in the plane and therefore the $z$ stability is not as much affected as the stability in the plane. As one would expect then, the vertical instability is simply dominated by the stochastic layers around the vertical axisymmetric resonances which are concentrated near the end of the bar, this is enhanced by the time dependency but not nearly as much as instability in the plane.

The above means that many orbits with large Liapunov exponent are trapped near the disk plane because the diffusion rate normal to the plane is small (or non-existent). Fig. 18 shows a segment of such an orbit. The most important characteristics of the motion are the large deflection angles as the trajectory moves in and out of the rotating potential of the bar on eccentric motion forced by the asymmetry of the halo mass distribution. This type of motion results in a large Liapunov exponent. There is no equivalent motion in the vertical direction, since the bar is flattened and rotates strictly in the disk plane.

The relative bar perturbation is much less (about half as) significant in Models 2 and 3 than in Model 1 and therefore one expects the fraction of orbits with large Liapunov exponents to be smaller in these models than in Model 1. This is easier to see in Model 2 where, in addition, by virtue of its less concentrated density distribution, many low order axisymmetric resonances 

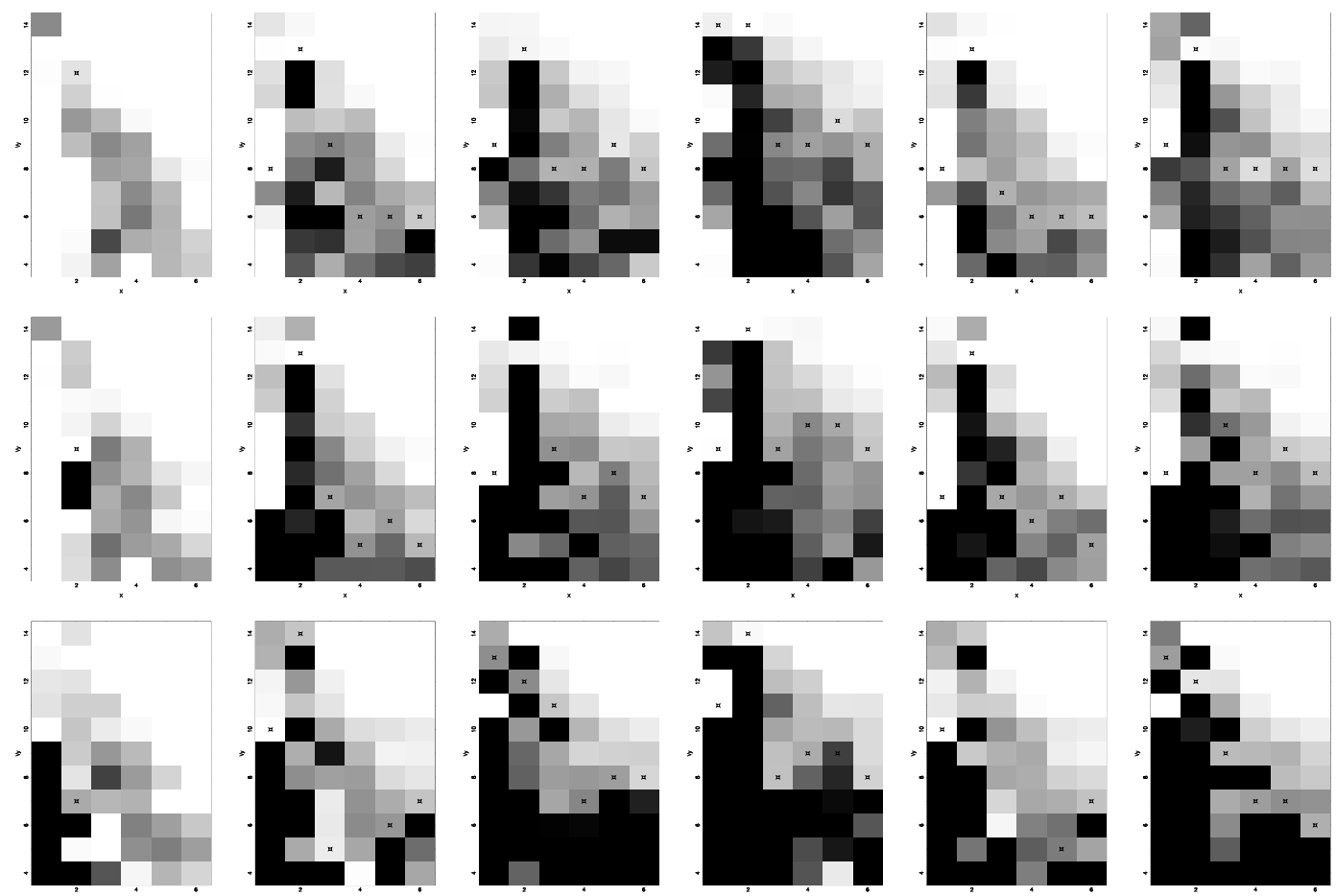

Fig. 19. Same as in Fig. 16 but for orbits of Model 2

are missing and others are deep in the core and thus have a limited effect. The Liapunov exponent greyshade diagrams for that model are shown in Fig 19. Again there are some orbits with large Liapunov exponents that are confined to the disk plane, this effect however was found to be less apparent in these models (especially in Model 3) than in Model 1 because of their lower asymmetry and the smaller bar contribution - causing the motion in the plane to be more regular. Clearly however, the simple relationship between the Liapunov exponent value and the $z$ excursion found in the runs in potentials without bars are not valid in any of the barred models discussed here. It is possible that evaluating the whole spectrum of Liapunov exponents and adding up the positive ones (to obtain the KS entropy: e.g., LL) may give a better approximation to the ergodic properties of the orbits. Chaotic two dimensional orbits must conserve some quantity at least approximately. This slow diffusion rate should correspond to a low value in one of the positive exponents. However this has not been checked here since we only calculate the maximal exponent.

The main processes described above are enhanced when one replaces the Ferrers bar with a homogeneous rectangular one. Namely, the maximal Liapunov exponents on average become much larger but the correlation between the values of these quantities and the maximum $z$ excursion becomes worse. The greyshade diagram for Model 1 with an added rectangular bar are shown in Fig. 20. Chaotic trajectories are found to be more common and the exponentiation time-scales are very small (of the order of a dynamical time). This is of course because of the much more asymmetric shape of the bar, which causes 

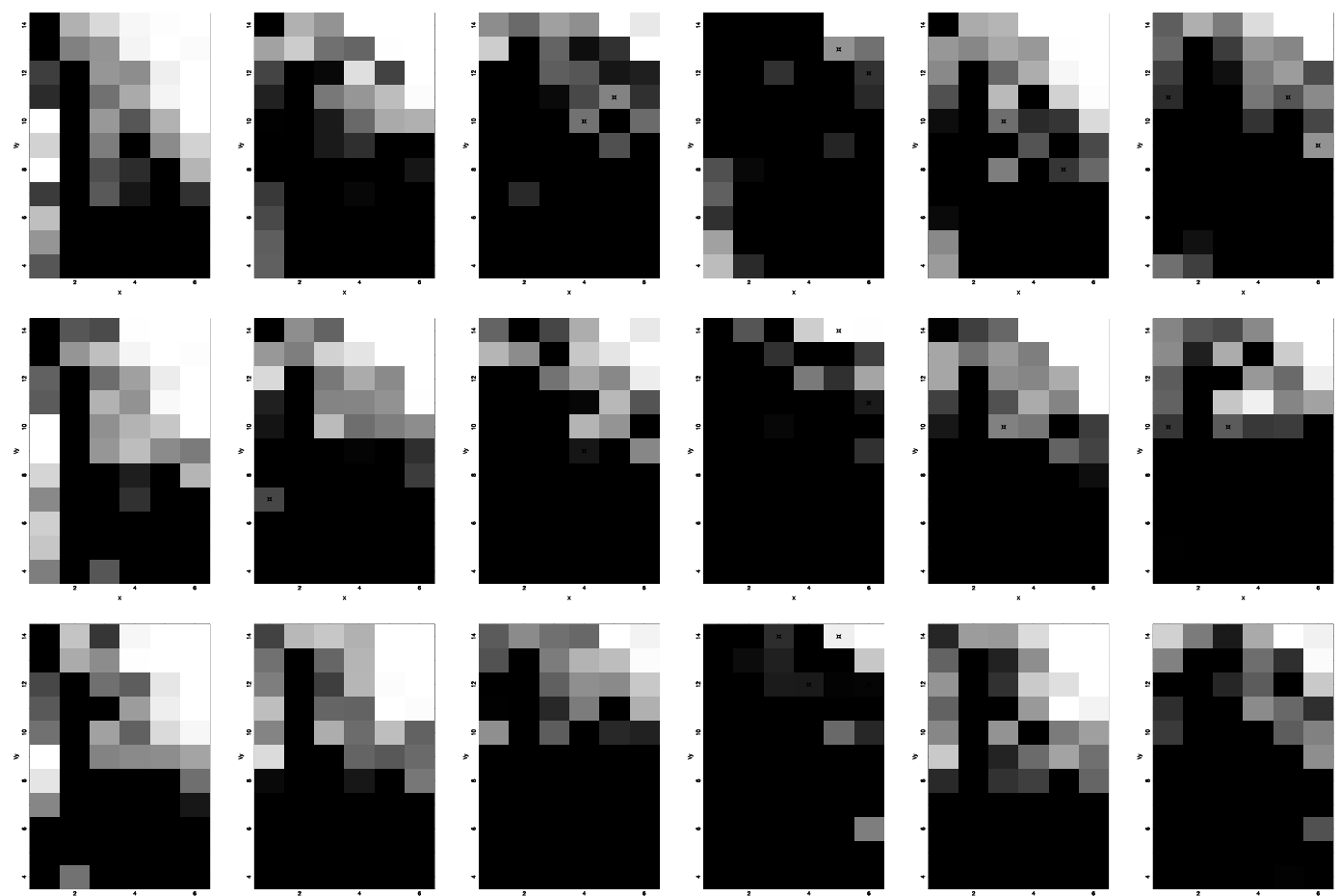

Fig. 20. Same as in Fig. 10 but when a homogeneous rectangular bar with the same parameters as the Ferrers bar replaces it

even larger and more frequent large angle deflections than the ellipsoidal Ferrers bar. The exceptions to this were the runs where a spherical halo was present and a central mass concentration is added. In this case, the potential is not time-dependent, thus there is no mechanism favouring instability in the plane.

Similar results to those described above were found for runs of Models 2 and 3 with rectangular bars. However, as in the Ferrers bar case, the values of the Liapunov exponents for these models were generally found to be somewhat smaller and with isolated regions of stability more common. On the other hand, contrary to the Ferrers bar case, the discrepancy between the values of the Liapunov exponents and the $z$ excursions in Models 2 and 3 was found to be somewhat larger than in Model 1.

\subsection{The effect of weak dissipative perturbations}

In addition to stars, the motion of which is essentially determined by gravity, galaxies also contain a gaseous component. Modelling the interstellar medium is not a trivial task. There are various processes that are thought to take place simultaneously in this medium. Among these are heating processes due to supernova explosions, the degradation of ordered motion through viscous processes and the inverse cooling processes which lead to heat being radi- 
ated away (e.g., Spitzer 1978). These processes appear to act in a complex and highly non-uniform manner, leading to several phases with very different properties (e.g., Norman \& Ikeuchi 1989; Clifford 1983).

One important characteristic that has to be taken into account when attempting to model the interstellar medium is its highly clumpy and apparently nonuniform nature. This property renders standard hydrodynamical treatments based on the continuum approximation and a perfect gas equation inadequate, since the assumption of local thermodynamic equilibrium stemming from a certain separation of "fast" and "slow" processes is no longer satisfied in a straightforward manner. In view of the above difficulty, an alternative approach based on the dynamics of a collection of locally interacting "sticky" particles has been tried by many authors (e.g., Combes \& Gerin 1985). In this scenario, particles move influenced only by gravity until they come close enough together when they collide inelastically. Although such a procedure might seem at first sight artificial and somewhat trivial, it can actually be fairly rigorously justified under certain conditions if some refinements are introduced. The clumpy and apparently scale invariant nature of the gaseous interstellar medium has also inspired attempts to treat it as a fractal object (Pfenniger \& Combes 1994). This method still awaits detailed application to realistic situations.

Some of the issues mentioned above are discussed in a little more detail in Section 4.5. We just mention here that there are indeed fast and slow processes which can be separated. These are the collision time of individual gas clouds, which is of order of 1 to $10 \mathrm{Myr}$, and their dynamical time which is much longer - being of the order of 60 to $600 \mathrm{Myr}$. This feature enables one to reconcile the two approaches (continuum approximation and sticky particle techniques) described above. (Details of the grounds on which such an approach may be justified are given by Scalo \& Struck-Marcel 1984). In this new formulation, one considers the hydrodynamics of collections of gas clouds which interact with each other over time-scales which are short compared to the dynamical time. A new set of hydrodynamic equations more appropriate to this situation is thus obtained. In these equations a "fluid element" is therefore a region small enough so that the macroscopic gravitational field can be considered roughly constant while large enough to contain a fair sample of gas clouds.

According to Combes (1991), the total mass of molecular Hydrogen in the Milky Way, for example, is about $2-3 \times 10^{9}$ solar masses concentrated in clouds of mass greater than $10^{5}$ solar masses. At about $10 \mathrm{kpc}$ these are concentrated in a region of a hundred pc from the plane of the disk. Therefore, the volume mentioned above would be of the order of, say, 500 by 500 by 100 pc, but of course will be smaller in the central areas where the concentration of molecular hydrogen increases significantly (Combes 1991). 
Assuming that the hydrodynamic effects (e.g., pressure, viscosity etc.) are small (i.e., gas clouds move primarily under the influence of gravitational forces) and that any evolutionary effects are slow, one can imagine the full hydrodynamic equations to be perturbations to the Collisionless Boltzmann Equation. This approach is similar in nature to the representation in Section 4.2 of discreteness noise in the $N$-body problem as a perturbation to single particle trajectories. Here we will follow Pfenniger \& Norman (1990; hereafter $\mathrm{PN}$ ) and assume that in the context discussed above, the main hydrodynamic effect can be approximated to first order as a velocity dependent viscous force, the value of which is fairly small in comparison to the value of the mean gravitational field. In PN, dissipative perturbations of the form

$$
F_{\text {fric }}=-\gamma v \mathbf{v}
$$

where $\gamma$ is a constant which determines the strength of the dissipation, were used. This form is similar to the form obtained if one assumes that the individual clouds are composed of infinitely compressible isothermal gas. Then in each collision the acceleration during an encounter of two clouds is given (e.g., Quinn 1991) by

$$
a_{e n} \sim u^{2} / r
$$

where $u$ is the absolute value of the relative velocity of the clouds and $r$ is their separation.

One can, to first order, estimate $\gamma$ using the usual "mean free path" approximation of (linear) viscosity:

$$
\gamma=n \lambda v_{r m s}
$$

where $n$ is the volume number density of gas clouds, $\lambda$ the mean free path and $v_{r m s}$ is the average random velocity. Supposing that the volume density is of the order of a few hundred or so per cubic kpc and the random velocity of the order of a few parts per hundred kpc/Myr, so that the mean free path is also of that order if the mean free time is about 1-10 Myr. This gives a value $\gamma \sim 0.01-0.1$. Following $\mathrm{PN}$ we use more conservative values for $\gamma$ and see that even these can have highly non-trivial effects.

\subsubsection{Dissipation in disk-halo systems}

Using a value of $\gamma=0.005$ we have integrated the equations of motion of dissipative particles moving in potentials with disk and triaxial halo contributions. The results are easier to interpret (and the figures in general tidier) in the case 


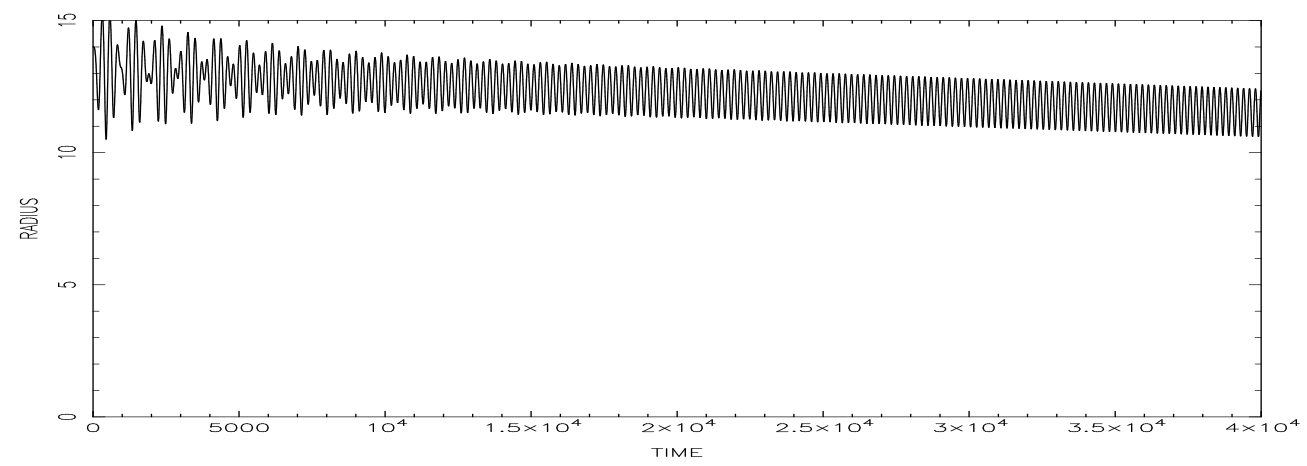

Fig. 21. Evolution of radial coordinate of trajectory starting from an initial value of $14 \mathrm{kpc}$

when a halo alone is present, although the situation is qualitatively the same when an additional disk contribution is also present (as long as significant asymmetry remains of course). We stick here to the case $G M_{D}=0$ and the other parameters being those of Model 2 with $c / a=0.8$ and $b / a=0.9$. All trajectories studied start on the $x$-axis with the local rotation velocity in the azimuthally averaged potential.

The quantity $\mathbf{v}$ in Eq. (21) is taken to be the vector difference between the velocity of the test particle and that of a particle moving with the local circular velocity. Such motion cannot exist because in a non-axisymmetric potential the orbits with the smallest radial departure from circular motion are ovally distorted closed loop orbits. Because the dissipation rate is small however, far from the core where these orbits are fairly close to circular, the difference in velocities is fairly small and trajectories can oscillate around the closed loops for very long times (Fig. 21). This means that, effectively, our dissipative point particles representing the fluid elements of gaseous disks, settle into regular quasiperiodic "limit cycles". In the case where we would have calculated $\mathbf{v}$ in terms of the difference between a particle's trajectory and the local periodic loop orbit, we would obtain a true limit cycle as the asymptotic attractor (an excellent review of concepts related to chaos in dissipative systems is given by Eckmann \& Ruelle 1985).

The situation is rather different however as one moves nearer the halo core. In this case, the closed loop orbits become more and more eccentric and cannot parent any trajectories that spend all their time inside the core (Fig. 22). One can then say that the above attractor ceases to exist, leaving only the center as a stable attractor for trajectories inside the core.

In Fig. 23 we plot the radial coordinate of a trajectory starting at $6 \mathrm{kpc}$. As can be seen, as one moves closer and closer towards the centre, the dispersion in the radial coordinate of the particle increases as the local loop orbits become more and more eccentric. At the separatrix beyond which no loop general loop orbits can exist (which occurs at about $4 \mathrm{kpc}$ ), there is a sharp transition in 

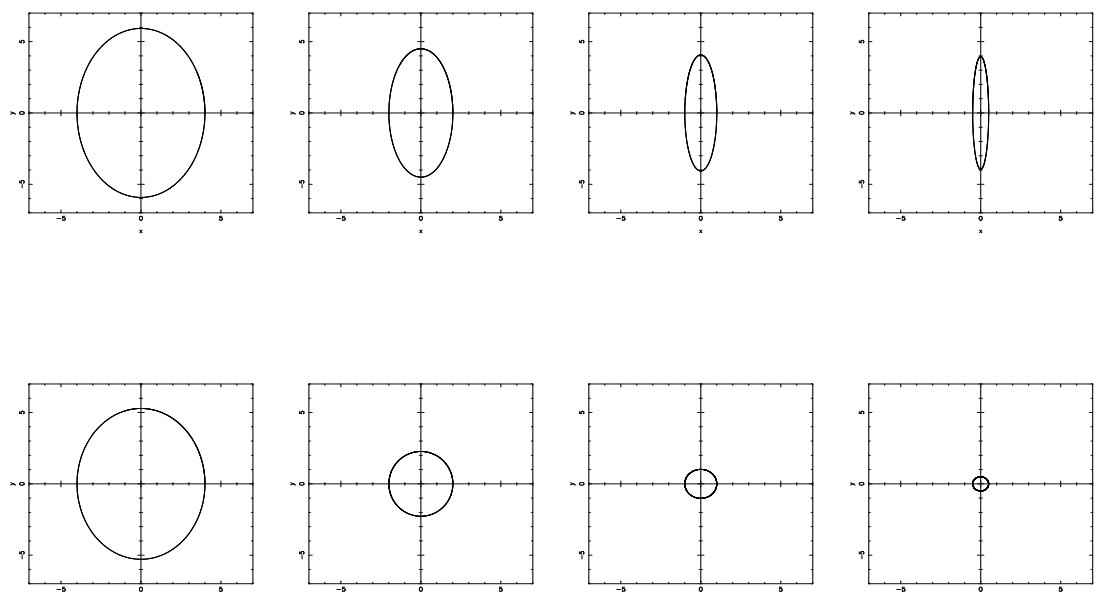

Fig. 22. Sequence of closed loop orbits. Top: When no central mass is present. Bottom: In the presence of a central mass $G M_{C}=0.01$

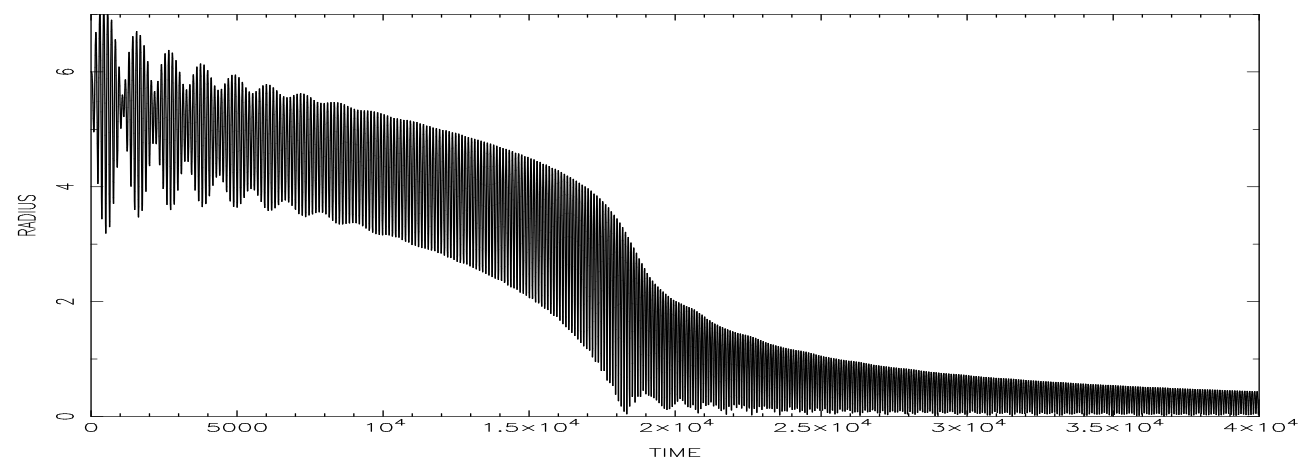

Fig. 23. Evolution of radial coordinate of trajectory starting from an initial value of $6 \mathrm{kpc}$

the dissipation rate and the particle quickly moves towards the centre.

Fig. 24 displays the spatial evolution of this trajectory, which shows it to follow a sequence of closed loop orbits followed by what essentially are sections of box orbits. This latter behaviour, which starts roughly at about $16000 \mathrm{Myr}$ and increases the dissipation rate dramatically, is shown in the plot on the right hand side of this figure. This process of course will lead to the growth of central mass concentrations, which as we have seen, can be important in determining the orbital structure. However it appears that it slows down significantly as the central mass increases. The central mass ruins the harmonic nature of the potential core which also becomes more symmetric in the inner regions so that the closed loop orbits, which now exist in the central areas, are close to circular (Fig. 22). The extent of the region where the potential is strongly affected by the presence of the central mass will of course depend depend on its strength. If the central mass is strong enough, this will be the whole harmonic core and nearly circular loop orbits could exist everywhere in the central areas.

The top diagram in Fig. 25 shows the evolution of the radial coordinate for 

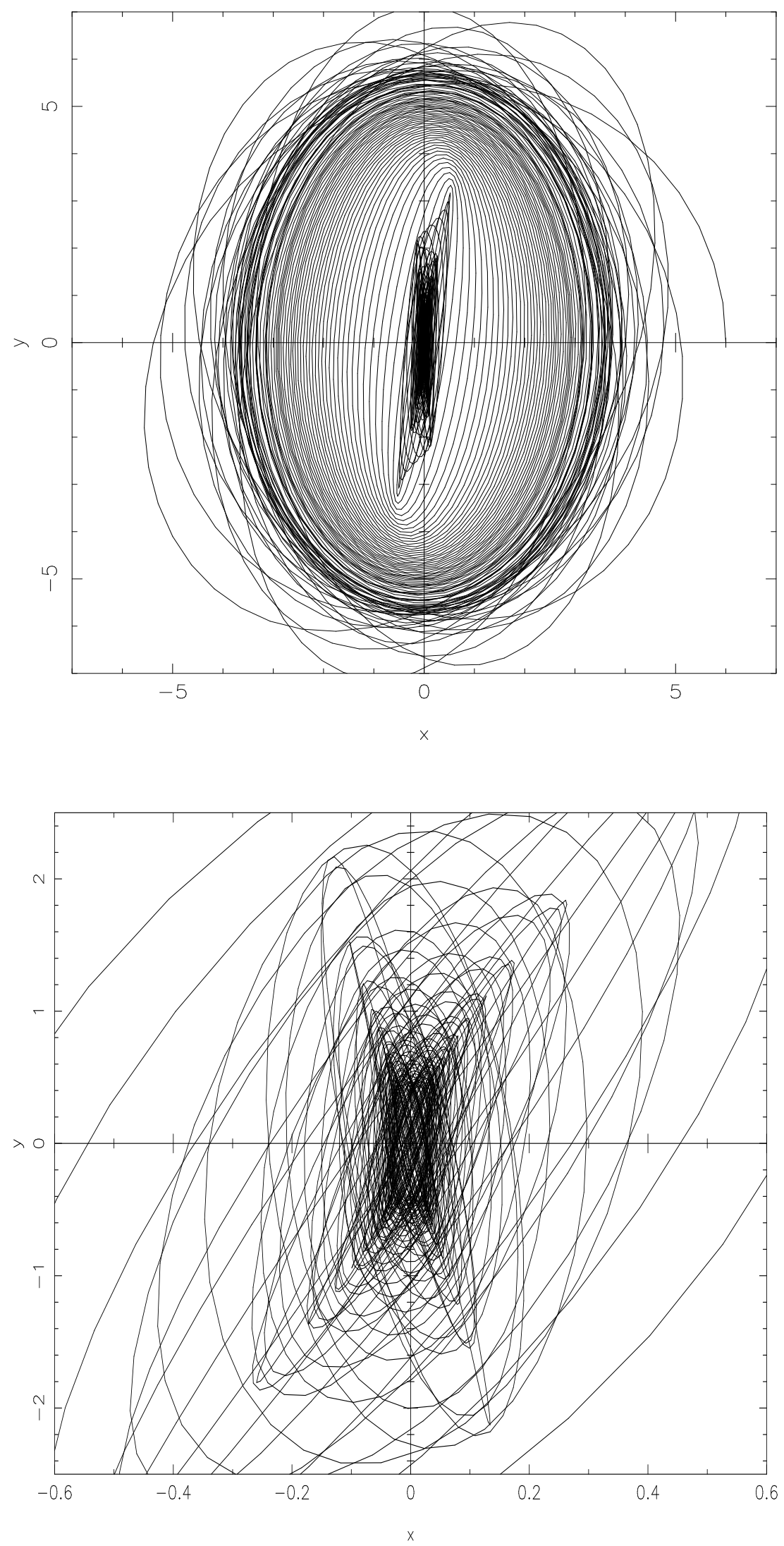

Fig. 24. Trajectory in the $x-y$ plane of orbit with radial coordinate represented in Fig. 23 and a more detailed view of the segment of the trajectory from $1750 \mathrm{Myr}$ to end of the run at $50000 \mathrm{Myr}$ (bottom) 

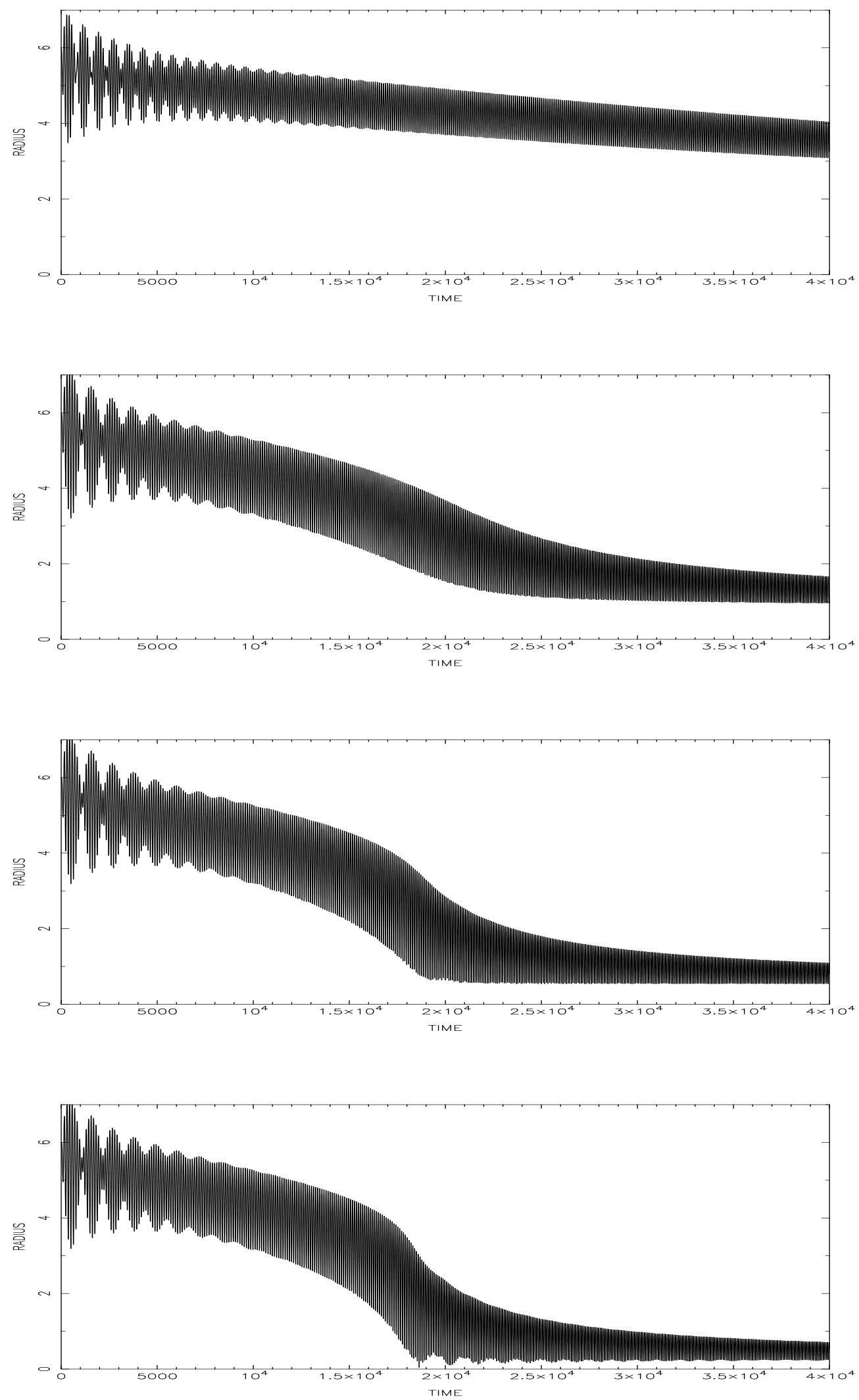

Fig. 25. Same as in Fig. 23 but with a central mass of (from top to bottom) $G M_{C}=0.01, G M_{C}=0.0005, G M_{C}=0.0001, G M_{C}=0.00001$ 
a trajectory starting from the same initial conditions as in Fig. 23 and exactly the same potential except that a central mass of $G M_{c}=0.01$ is present. Clearly, the behaviour previously observed — that is the accelerated dissipation rate - is no longer present. For very small central masses (anything greater than $G M_{C} \sim 0.00001$ ), loop orbits exist deep inside the core. Subsequent plots of Fig. 25 clearly show that there is a certain region, between the halo core radius and the centre, where the trajectories are extremely eccentric and the dissipation is very large. The scope of this region increases with decreasing central mass. Orbits completely confined in this annulus cannot be parented by the closed loops, therefore they can either oscillate around the variety of boxes and boxlets available or move chaotically. This causes the large increase in the dissipation rate. If the central mass is strong enough, closed loops exist everywhere and so this region does not exist - which means that the dissipation rate is always small. Thus there is a threshold for the central mass concentration beyond which accretion completely stops - and does not appear to be above a few tenths of a percent of the relevant total mass (e.g., within 10-20 kpc).

It is also possible for dissipative orbits to be $z$ unstable. However, given the fact that the instability requires significant central mass and that in this case trajectories settle down on closed loops, few orbits are $z$ unstable unless the dissipation rate is very small. Nevertheless, we expect the process of accretion of material to the central region (in a realistic situation) to be accompanied by shocks which may cause much of the gas to be transformed into stars (e.g., Pfenniger 1993). These will be born on eccentric trajectories which will usually be vertically unstable as we have seen in Section 4.1. As the central mass increases, one expects this to take place at progressively larger radii (as the accretion from the innermost region stops and trajectories with higher energy are destabilised by the growing central concentration). Once the harmonic core is completely destroyed, the accretion ceases completely, and all box orbits within the core are also destroyed. One therefore expects the formation of a bulge-like structure, the extent of which is similar to that of the original harmonic core, and which is formed "inside out" from layers of unstable trajectories. Since however the inflow of mass is gradual and saturates before a situation is reached whereas most box orbits are unstable over short time-scales (cf., Section 4.1 Merritt \& Quinlan 1997) the above processes are expected to be slow. In particular, a significant bulge may form before the halo loses triaxiality (if at all).

\subsubsection{Dissipation in systems with rotating bars}

When the halo is axisymmetric, the effect of dissipation is analogous to the models studied by $\mathrm{PN}$ - in regions where stable $x_{1}$ periodic orbits exist, the dissipative trajectories mimic these, however in the case the $x_{1}$ are unstable, 


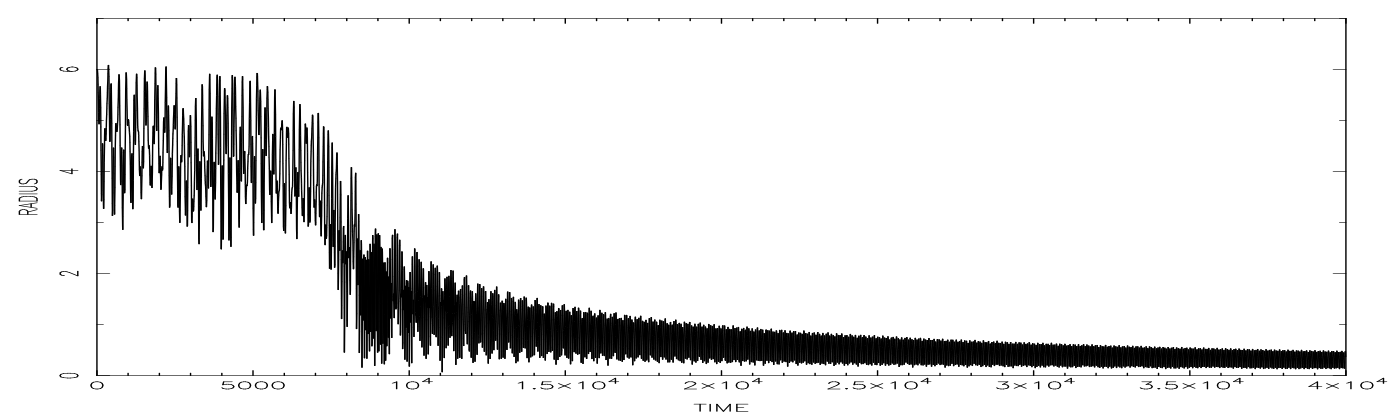

Fig. 26. Trajectory started from edge of the bar in Model 2 with axis rations $b / a=0.8$ and $c / a=0.7$
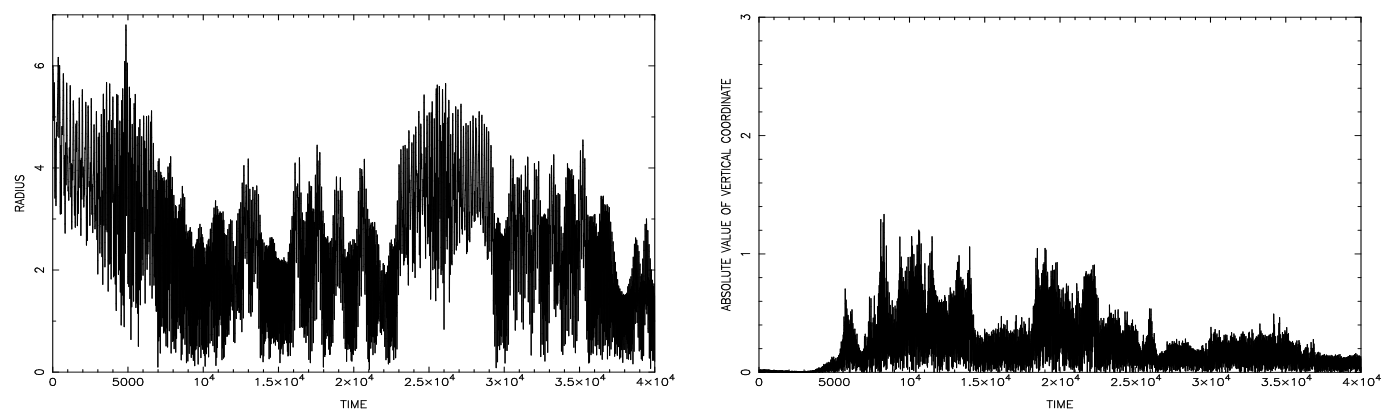

Fig. 27. Left: Same as in Fig. 26 but with $\gamma=0.0005$. Right: Corresponding evolution of the absolute value of the $z$ coordinate

the dissipative trajectories are quickly brought inwards, a process that is accompanied by (modest) $z$ instability. The addition of a central mass brings about large instability strips in the $x_{1}$ family, especially in the central areas $(\mathrm{PN})$. (This is in contrast to the case when no bar is present, when the central mass actually stabilizes the closed loop orbits!) The effect of orbit dissipation therefore becomes much more dramatic with an attracting point at the centre quickly reached. Varying the flattening of the halo has the only effect of suppressing the $z$ instabilities even further (since the vertical resonances are moved in).

We focus on the case where the halo is non-axisymmetric. We will concentrate on trajectories of Model 2 which has its rotation curve rising over the extent of the bar's major axis (an observed property of bars: e.g., Pfenniger 1984a) and a large axisymmetric disk contribution. Indeed, this model appeared in the Hamiltonian limit to have a more regular phase space than the other two models - thus suggesting that it may be possible for a bar to survive long enough so that its effects on the gas dynamics be important (see Section 4.3 

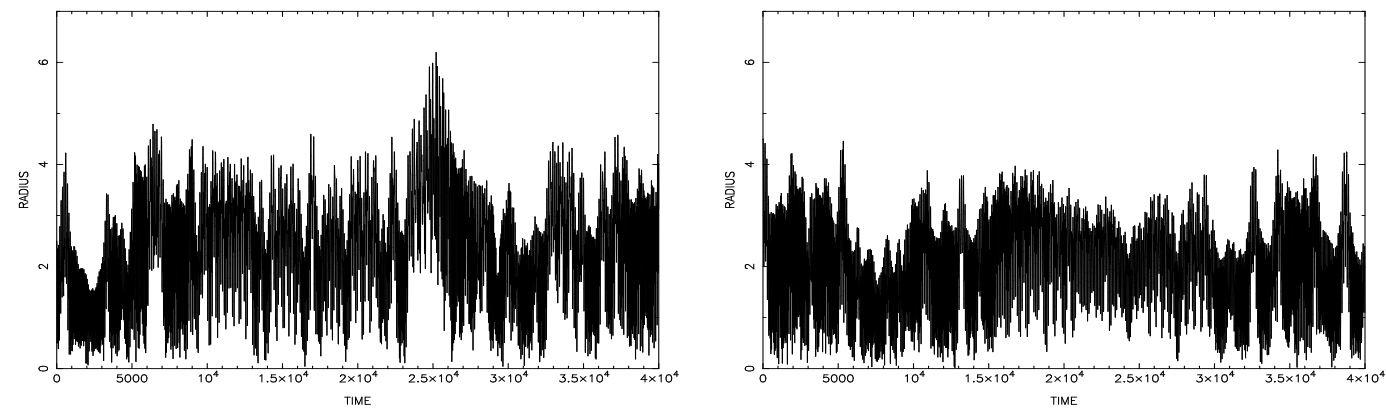

Fig. 28. Evolution of radial coordinates for trajectories starting at a only $2.5 \mathrm{kpc}$ (left) and $4.5 \mathrm{kpc}$ from centre of the mass distribution but appearing to end up in the same attractor as the trajectory in Fig. 27. Here also $\gamma=0.0005$

above). We fix the halo potential axis ratio in the disk plane at $b / a=0.8$ while normal to the plane of the disk we take the axis ratio to be $c / a=0.7$.

Fig. 26 shows the time evolution of the radial coordinate of a particle started from the edge of the bar with the velocity of a circular orbit in the azimuthally averaged potential. As can be seen from that figure, even trajectories starting this far out from the centre of the potential can be transported there. It can be noted however that after the initial rapid decay in the radial coordinate, the trajectory settles down to a state where this coordinate oscillates about a value of roughly $0.5 \mathrm{kpc}$ with little further decay. It is not obvious whether this is a regular limit cycle or a strange attractor. In principle this can be checked by calculating the Liapunov exponents for example, though the difference here is of no great practical consequence. However, when the dissipation was decreased (by lowering the value of $\gamma$ to $\gamma=0.0005$ ), it was found that some end states can be seen to be highly chaotic even by simple inspection. Fig. 27 displays the radial coordinate time series of such an orbit. This behaviour is also usually accompanied by non-negligible excursions in the vertical direction which are suppressed by high dissipation rates. It is also interesting to note that trajectories starting from as small an initial radial coordinate as $2.5 \mathrm{kpc}$ appear to end up in this attractor, at least as far as the radial coordinate behaviour is concerned (Fig. 28).

In the presence of a stronger bar and in the absence of dissipation (e.g., $G M_{B}=$ 0.04 ), it was found that many of the trajectories starting near the end of bar escape in the Hamiltonian limit. In the presence of significant dissipation however most of these trajectories end up in stable attractors outside the bar.

\subsection{Gas dynamics}

The dissipative point particle approach used above may give some indication about the effect of dissipation but is certainly not suitable for detailed work. 
The best numerical approach to modelling clumpy structures such as gas cloud systems in galaxies is the kinetic approach.

The gas in galaxies moves mainly under the influence of gravity. However when gas clouds, or groups of gas clouds, come within a certain distance of each other, non-gravitational forces such as viscous and pressure forces act. Pressure forces act to scatter the clouds off each other, while viscous forces act to cause dissipation (only the latter were considered in the point particle case). Thus we have the standard weak fluctuation dissipation behaviour described by the Fokker-Planck Equation. The Fokker Planck equation is a partial differential equation of seven variables and is thus not easily solved directly in the general case. However, one can set up numerical experiments that mimick the behaviour expected of the evolution ruled by this equation. A simple algorithm displaying the Fokker Planck kinetic behaviour was developed by Hoogerbrugge \& Koelman (1992). We describe it here in language adapted to our situation (instead of that used in conjunction with laboratory systems for which the method was developed).

In the aforementioned algorithm, particles move in the smoothed out galaxy potential for a time interval $\Delta t$ after which a search is made to identify the nearby neighbours $j$ of each of the particles. At that point, the velocity of the $i^{\text {th }}$ particle is updated according to

$$
\dot{\mathbf{x}}_{i}=\dot{\mathbf{x}}_{i}+\sum \Omega_{i j} \mathbf{e}_{i j}
$$

where $\mathbf{e}_{i j}=\left(\mathbf{x}_{i}-\mathbf{x}_{j}\right) /\left\|\mathbf{x}_{i}-\mathbf{x}_{j}\right\|$. Linear and angular momentum conservation require that $\Omega_{i j}=\Omega_{j i}$, and that it is a scalar function of the relative positions and velocities of the particles. A particularly simple function satisfying these conditions is a direct modification of the one used by the original authors, but facilitating direct comparison with our dissipative point particle simulations and conditions in the interstellar medium (Section 4.4.1)

$$
\Omega_{i j}=\Theta_{i j}\left(\Pi_{i j}-\gamma_{p}\left\|\dot{\mathbf{x}}_{i}-\dot{\mathbf{x}}_{j}\right\|^{2} \mathbf{e}_{i j}\right)
$$

where $\Theta=1$ when the separation between the two particles is less than a certain radius $r_{i n t}$ and zero otherwise. $\gamma_{p}$ determines the strength of the dissipation. $\Pi$ is sampled from a Gaussian distribution with mean $\Pi_{0}$ and dispersion $\delta \Pi$. Given that these collisional processes are fast compared to a dynamical time, we expect that hydrodynamical behaviour is recovered on large scales. Indeed, it can be rigorously shown that this is what the algorithm reproduces when the above condition is satisfied (Espagnol 1995). In this case we have a stable local thermodynamic equilibrium if the "generalized Einstein 
relation" (e.g., Klimontovich 1994) is satisfied. In our case this means

$$
\Pi_{0}=\gamma_{p}\left\|\dot{\mathbf{x}}_{i}-\dot{\mathbf{x}}_{j}\right\|\left(\left\langle\left\|\dot{\mathbf{x}}_{j}\right\|^{2}\right\rangle-\left(\left\langle\dot{\mathbf{x}}_{j}\right\rangle\right)^{2}\right)
$$

where the local mean squared velocity is averaged over the neighbours interacting with particle $i$.

In practice the right hand side of (26) should be multiplied by a factor smaller than 1 to have predominantly dissipative behaviour. In the simulations we now describe this was taken as 0.9 . Also, since we use a relatively small number of particles (500), we just use the heuristic relation

$$
\Pi_{0}=\gamma_{p}\left\|\dot{\mathbf{x}}_{i}-\dot{\mathbf{x}}_{j}\right\|^{2}
$$

which makes the fluctuation term of similar form to the dissipation term. In this case the dissipation term of Eq. 21 is related to $\gamma_{p}$ simply by

$$
\gamma \times \Delta t \sim(1-0.9) \gamma_{p} \times \sqrt{N_{\text {int }}}
$$

The interaction range (which scales as $\sim \sqrt{N}$ in a flat system) was also taken to be rather large to make up for the small number of particles: $r_{i n t}=0.5 \mathrm{kpc}$ . There were roughly $N_{\text {int }}=4-8$ particles within that radius for each particle. $\Delta t$ was taken to be $5 \mathrm{Myr}$. We therefore take a value of $\gamma_{p}=0.12$ to match the value $\gamma=0.005$ usually adopted in Section 4.4. Finally the dispersion of the Gaussian distribution was taken as $\delta \Pi=0.2 \Pi_{0}$.

The particles are placed at random in the inner $10 \mathrm{kpc}$ of a given model. A hundred of them have $z=0$ and the rest are in groups of a hundred with $z=-0.2,-0.1,0.1,0.2$. All particles are given the local rotation velocities in the plane. Fig. 29 shows the results for the halo models in which the dissipative point particle runs of Figs. 21 and 23 were integrated. Clearly, one recovers the same conclusions as before: trajectories inside the core radius of the halo quickly dissipate and spiral down towards the centre, while those in the outer areas find stable loop orbits to oscillate around and are relatively long lived. Fig. 30 shows the case when a central mass $G M_{C}=0.01$ is present. Again, just like in the case of single dissipative point particles, no dissipation towards the centre takes place (cf. Fig. 25).

Modelled in a self consistent way (with the gravitational field of the gas taken into account), this process will lead to a self regulating mechanism for gas accession to the central regions of a galaxy. In a general situation the particles should also be assigned different sizes, masses and "internal energies" which they can exchange with each other during collisions. This might enable one to 

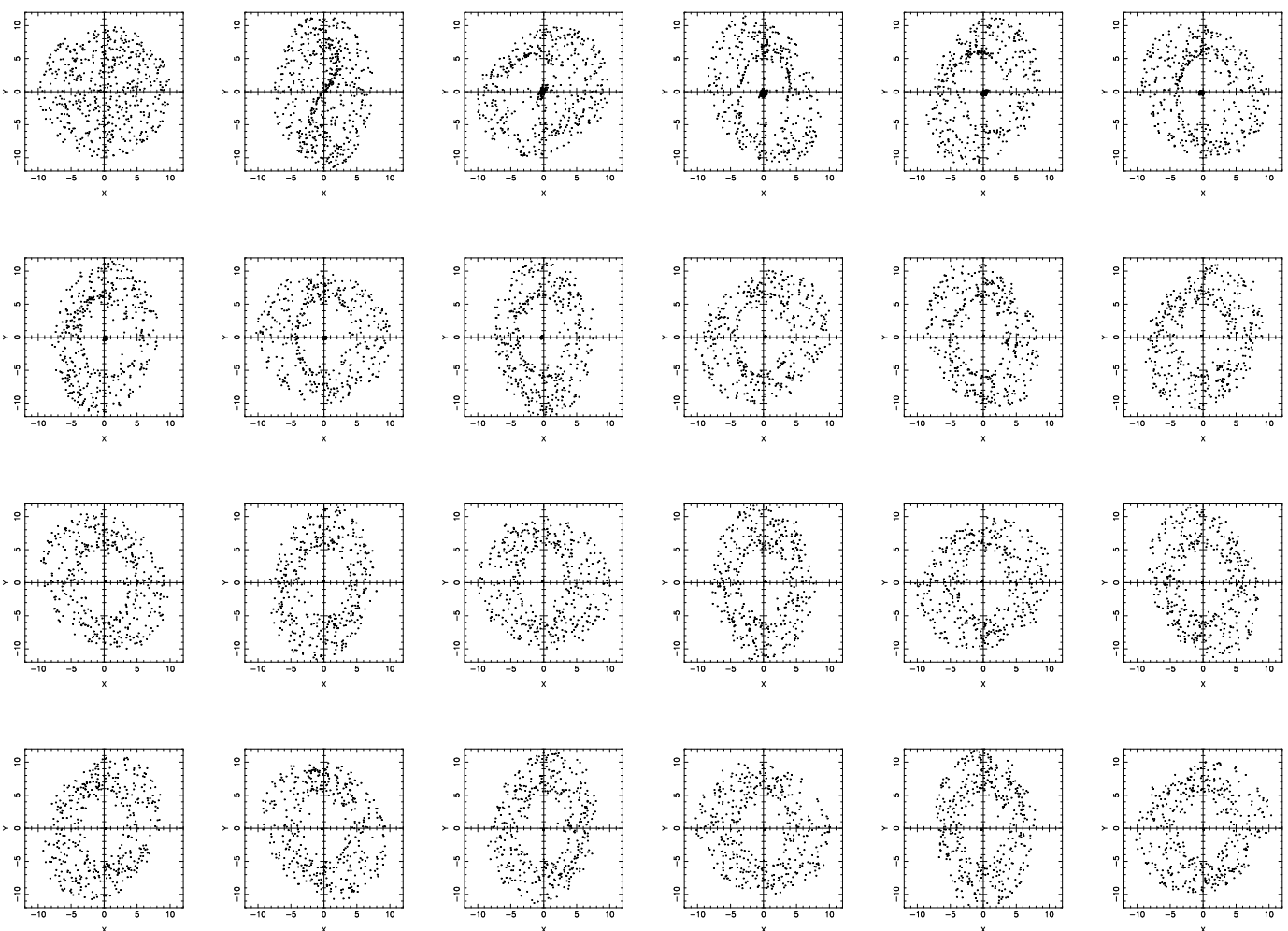

Fig. 29. $x-y$ projection of spatial evolution of Fokker-Planck type sticky particle system in model corresponding to the plots in Figs. 21 and 23. Snapshots are shown at intervals of $500 \mathrm{Myr}$ (in sequence from left to right and from top down)

model "physical" processes such as fragmentation of gas clouds, coalescence, star formation and energy feedback into the interstellar medium, which are now believed to have an important effect on the dynamics and which should be included in any detailed modelling. However, it is clear that to a first approximation, the main effect of a triaxial potential with a harmonic core is to provide an efficient self regulating mechanism for accretion of gaseous material within that core to the central region. This is not only important for the formation of central masses which are so crucial in determining the dynamics, but perhaps to other phenomena like the fueling of active galactic nuclei. It provides an alternative mechanism whereby non-axisymmetric perturbations can lead to gas accretion into the inner areas of galaxies, now that the correlation between the presence of galactic bars and of AGN in galaxies is thought to be weak (e.g. Mulchaey \& Regan 1997). The fact the accretion is takes place over a Gyr and is self regulating makes it compatible with the lifetime of AGN and with the observation of Merritt \& Quinlan (1997) that observationally detected black holes have smaller masses than the critical mass required to destroy axisymmetry — the more obvious mechanism for self regulation. 

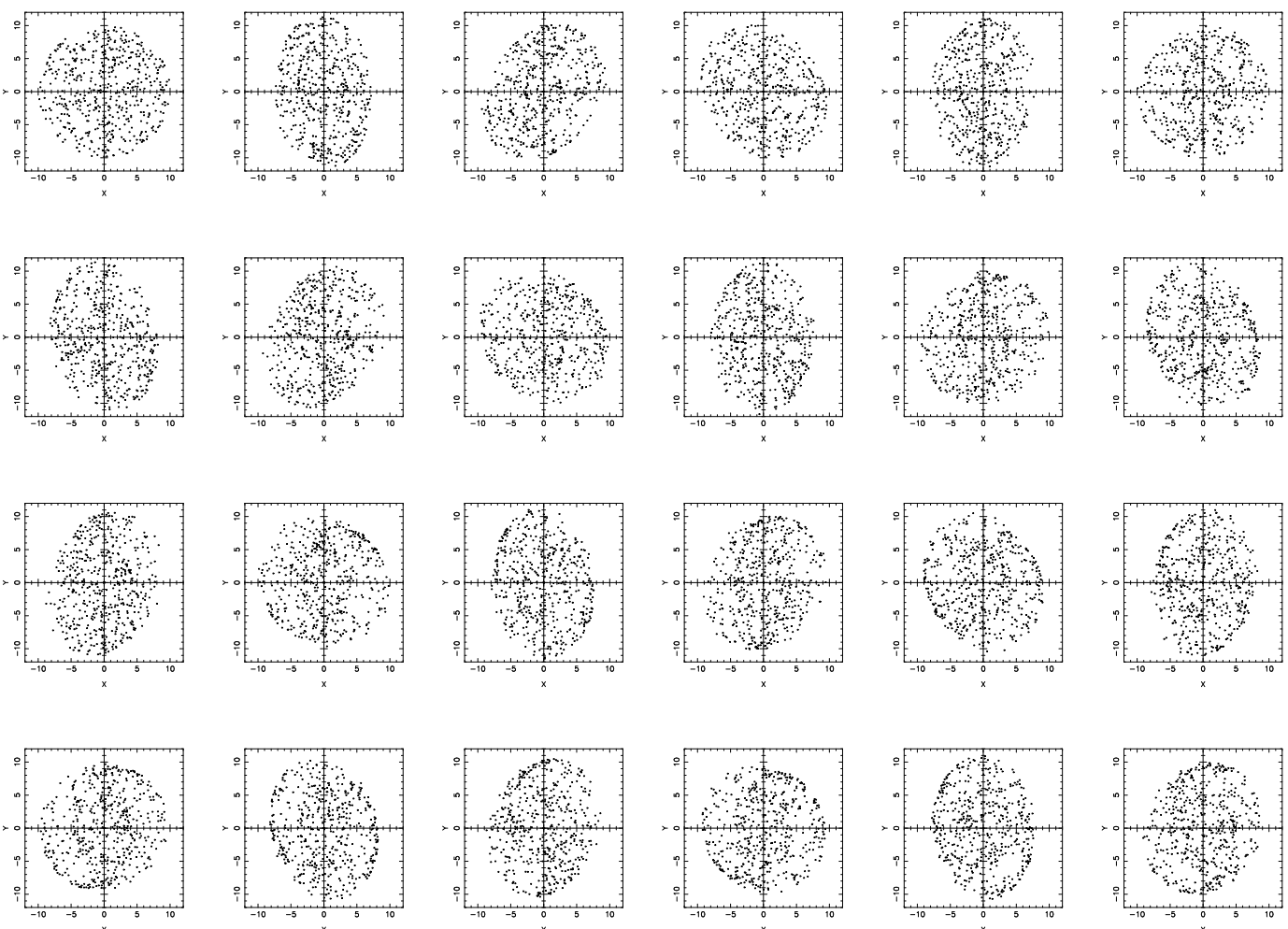

Fig. 30. Spatial evolution of sticky particle system of Fig. 29 when a central mass is added. Snapshots are shown at intervals of $500 \mathrm{Myr}$ (in sequence from left to right and from top down)

\section{Conclusions and possible consequences}

\subsection{Summary of results}

Triaxial structures are expected from the instability of gravitational collapse to non-spherical perturbations and the ineffectiveness of violent (or collisionless) relaxation in washing out completely the initial state. Triaxial dark matter halos are predicted by hierarchical theories of galaxy formation and although there is some doubt if they could survive dissipational collapse, it seems that this would bring about at most a rearranging of shape but not complete loss of triaxiality. Here we have mainly concentrated on models with constant density core halos. These are favoured by observations but are not predicted by cosmological simulations which nevertheless are in agreement with other observed features (Section 2.1).

We have studied the orbital structure near the disk plane of disk galaxy models with triaxial halos. This was done by examining the vertical stability and calculating the maximal Liapunov exponent of general orbits and studying the stability of periodic orbits that parent them. These effects were, as could be 
expected, in general correlated. There are three factors which mainly determine the orbital structure of our models: the non-linearity of the force field, the asymmetry in the density distribution (Section 3.1) and the presence of a rotating perturbation (Section 4.3). The nonlinearity is determined by the central concentration of the density distribution. In models with core radii of $R_{0}=2 \mathrm{kpc}$ significant instability is found around the 1:2 resonance of the $x$-axial orbit, which is moved inwards when the asymmetry is greatest. For models with very small core radius, widespread instability occurs even in the axisymmetric case. For models with larger core radius or which were disk dominated in the central areas, the major low order resonances were too far out to be significant even when significant asymmetry is present.

One way of enhancing nonlinearity is by adding a central mass concentration. Then, as is usually the case (e.g., Gerhard \& Binney 1985), almost all box orbits are destabilised and replaced by either boxlets or chaotic orbits. The effect of the central mass can be understood in terms of the stability properties of the closed periodic orbits. Although the lower order resonances (e.g., the 1:2 resonance) appear at somewhat smaller radii when a central mass is present, the main effect causing the instability seems to be the creation and broadening of higher order resonances. When the central mass is strong enough, the instability gaps on the $x$ axis orbit merge and, even for relatively small central masses of about $10^{-5}$ the total galaxy mass at $20 \mathrm{kpc}$ the axis orbits (which parent the boxes) are destabilized and are replaced by higher order (KAM islands known as) "boxlets" (Section 3.1). Increasing the central mass destabilizes these and the remaining stable orbits are mainly those of still higher order in the self replicating phase space hierarchy (e.g., LL). This leads to widespread chaotic behaviour in the region of the phase space once occupied by the box orbits. In addition, it was found that the destabilisation of the box orbits takes place out to many core radii. Therefore high energy stars born on eccentric orbits may be also be unstable. For central masses of up to $0.05 \%$ the total galaxy mass at $20 \mathrm{kpc}$ many trajectories in the region once occupied by the box orbits are either regular or have small Liapunov exponents (and therefore also small diffusion rates) and thus any resulting evolutionary effects are expected to be slow. For central masses of $\sim 1 \%$ the galaxy mass (at 20 kpc) most orbits in the aforementioned region are strongly chaotic and quickly relax to an invariant distribution (Section 4.1).

Not all unstable periodic orbits are "centrophylic" boxlets. In fact many higher order looplets (in particular the 2:2 loops) which have a definite sense of rotation and do not pass near the centre, may be unstable in the presence of a central mass concentration. As might be expected, the fraction of looplet orbits is greater in more axisymmetric potentials, and, in general, the fraction of vertically unstable orbits was found to be larger in flatter potentials. Thus, the presence of a disk may, in some situations, increase the fraction of unstable general orbits. For although there are fewer box orbits to start with, many 
orbits with a definite sense of rotation (which are parented by higher order looplets) are unstable in the central areas and fewer orbits are parented by stable boxlets.

Even for highly asymmetric systems with strong central mass concentrations, and in the region of phase space once occupied by the box orbits, there remains a significant fraction of stable periodic orbits (albeit parenting a small fraction of general orbits). This will mean that even though there is widespread chaotic behaviour, trajectories in non-integrable but smooth galaxy potentials do not approximate strongly chaotic uniformly hyperbolic systems. Such systems therefore are not structurally stable and thus their qualitative behaviour can be affected by small perturbations (Section 4.2). This was indeed found to be the case. It was found that perturbations that take many Hubble times to change the energy can have a significant effect on the orbital structure. For smaller perturbations, the effect was more or less equally likely to stabilize the trajectories as to destabilize them. For stronger perturbations however the latter effect was much more predominant as these destroyed the intricate island structures. The fact that weak discreteness noise can have such an effect on the dynamics is important for both the estimation of the importance of discreteness effects in real systems and the faithfulness to which $N$-body simulations can reproduce the dynamics of galaxies. In particular, the triaxiality of the halos produced by $N$-body simulation should be taken as a conservative estimate since these simulations employ far fewer particles than present in halos mainly composed of elementary particles.

In the absence of rapidly rotating bar perturbations, the nearly circular closed loops are stable. This is due to the absence of resonances, which arises from the fact that the angular frequency and the $R$ and $z$ oscillation frequencies have nearly constant ratios in the type of models chosen (this is analogous to the situation when response is linear, in which case each of the frequencies is constant). The addition of a rotating bar breaks this symmetry and therefore markedly increases the fraction of chaotic orbits present - since now widespread chaotic behaviour can occur in both regions once occupied by the main orbit families (boxes and loops). The presence of the rotating perturbation within the triaxial halo potential means that the potential is time dependent and that no transformation of coordinates can change that. This will lead to very complicated behaviour near the disk plane, with trajectories suffering large angle scattering. The result is that trajectories are much more irregular in the disk plane where the time dependence is greatest. These trajectories are sometimes not $z$ unstable even though they may have a very large maximal Liapunov exponent. Thus some additional "invariant" may be at least approximately conserved. In this case therefore the maximal exponent does not provide complete information on the phase space transport properties. This was especially true if the bar has a rectangular shape. 
Central masses can be produced if small dissipative perturbations are given to the loop orbits in the inner areas near the halo core radius (where the 1:1 resonance occurs). Trajectories are then observed to spiral inwards in a few rotation times, even though the magnitude of the dissipation would require many Hubble times for this effect to be noticeable in the outer areas of the potential. This behaviour is easy to understand since closed loop orbits (which parent the general loop orbits) become more and more eccentric as one moves nearer to the center of the potential and do not exist at all deep inside the core, at the point where this happens (the "separatrix") dissipation rates are greatly enhanced. Beyond this, the trajectories do not find any closed loop orbits to oscillate around and quickly spiral to the centre. However once a strong central mass has formed, closed loop orbits are stabilised in the central area and dissipative trajectories may settle around them in stable limit cycles instead of spiraling to the centre. The build up of the central mass thus saturates. This provides a self regulating mechanism for the formation of central masses in galaxies, and may explain why black holes are observationally found to have masses smaller than those required to destroy the triaxial equilibrium (Merritt \& Quinlan 1997). Since a central mass of $0.05 \%$ of the total galactic mass at $20 \mathrm{kpc}$ appears to be enough to essentially stop further gas inflow. And thus from the discussion earlier in this section one expects the evolution caused by the central mass to be slow. The time-scale for the formation of the central mass is about 1 Gyr.

In the presence of time-dependent forcing (that is a rotating bar), dissipative trajectories were found to settle into long lived chaotic states where their radial coordinates oscillated around definite values. Such strange attractor states may explain some of the observed ring structure in galaxies ( $\mathrm{Gu}$ et al. 1996) - provided of course that a bar can survive long enough in a triaxial halo potential. Trajectories on some of these attractors may also have large $z$ excursions and large radial motion through the galactic disk.

\subsection{Possible consequences}

The effects described above may have the following consequences. First there will be a redistribution of the gas accompanied by the fuelling of a central mass. In general therefore, gas motion in nonrotating non-axisymmetric potentials may be linked to the fuelling of central black holes or trigger bursts of star formation as the gas moving on self crossing trajectories is shocked and dissipates towards the centre as in the case of barred potentials (Beckman et al. 1991; Pfenniger 1993). This provides an alternative mechanism now that the correlation between the presence of galactic bars and of AGN's is thought to be weak (Mulchaney \& Regan 1997). 
In the absence of a central mass, and within the region delimited by the harmonic core (approximately equal to $R_{0}$ if the halo is dominant but decreases with increasing symmetry), only box orbits can exist and almost all of these are unstable to perturbations out of the disk plane in the presence of a central mass concentration. Therefore, initially any stars born from a settling disk in this region will become unstable when a central mass forms. The vertical instability will lead to the formation of bulge like structures even if this system of stars, which is dynamically hot, is initially confined near the disk plane. As the central mass builds up, the harmonic nature of the potential is destroyed in its vicinity and closed loop orbits are stabilised at progressively larger radii within the core. Eventually, when the central mass is large enough, the harmonic core is completely destroyed. At that point the gas accretion also stops, since the gas can now settle on the newly created closed loops anywhere inside the core region (Fig. 25). One then expects the end result of this process to be the formation of a bulge-like structure, the extent of which is similar to that of the original harmonic core and is formed from layers of unstable trajectories forming from inside out (see the discussion at the end of Section reftax:dispdisk).

It then follows that haloes with larger cores will be expected to develop centrally condensed "bulgy" disks. Once fully evolved these will then be expected to dominate the rotation curve in the inner areas. On the other hand, for halos with smaller core radii, this effect will be less significant and the halo would dominate the rotation curve at all radii. This suggests an explanation as to why in galaxies with widely different disk-halo contribution rotation curves one still gets these to be approximately (but not exactly: Persic et al. 1996) flat over most of the detected radius of a galaxy. If this is the case then it will have to be that more massive haloes have larger core radii, since it is observed that galaxies in which are disk dominated near the centre have larger terminal rotation velocities. Since the effective harmonic core radius in the disk plane for a given $R_{0}$ is also a function of the asymmetry in that plane, one may also suggest that larger mass haloes may have a larger asymmetry.

We have seen that, in the presence of bars rotating in triaxial halos, almost all orbits become chaotic. In addition, these orbits do not have a definite sense of rotation. It is not clear if such a bar could form in the first place because of the large random motion (due to the triaxiality). However, if it is possible to still have a bar unstable disk inside a triaxial halo, it is likely to evolve a random bulge like structure extremely quickly as the bar is destroyed. It may be interesting to note here that observations from the Hubble deep field survey (van den Bergh et al. 1996) suggest that barred galaxies are rare at high redshifts. It is also found that the fraction of late type galaxies is much lower than in standard catalogues. This may be expected if disks form inside strongly triaxial haloes where star formation may be caused by gas particles moving in the central regions on self-intersecting trajectories. In the outer regions, 
gas particles may move around (non-self-intersecting) closed loop orbits so that the star formation rate is much smaller. In galaxies with small halo core radii (which according to our scenario would end up as late type galaxies), the more violent effects are limited to a small central region and the evolution will generally proceed at a much slower rate - which means less of them are observed at high redshift.

Evidently, as the above processes unfold, the halo gradually loses triaxiality (because of the destruction of the box orbits which are crucial for maintaining the triaxial structure, and the settling of stars onto loop orbits which are alligned perpendicular to the halo). This also can bring an end to the gas inflow and the scattering of stars into a bulge. This process is not expected to be very fast however, since for a weak central mass there is usually a large fraction of regular and weakly chaotic (ie., with small Liapunov exponents and phase space diffusion diffusion rates) orbits remaining in the region once occupied by the box orbits. The threshold central mass needed for the majority of trajectories in that region to be highly chaotic (i.e., to have an exponentiation time-scale of a dynamical time or so) appears to be much larger than the mass needed to stop the accretion. Thus, such central concentrations would probably never be reached because of the self regulating nature of the accretion mechanism, which may explain why central concentrations as high as those apparently required for rapid evolution towards axisymmetry in elliptical galaxies are rarely observed (Merritt \& Quinlan 1997). Therefore, one may expect there to be a period where the effects described above act on the stellar trajectories before the halo becomes completely axisymmetric (at which point it is then possible to develop a barred disk).

Although no precise determination of the time-scales of the processes described in this section can be obtained without full $N$-body simulation, including hydrodynamic effects, one can, to first order, perhaps give the following order of magnitude estimates. Since the time-scale over which originally planar trajectories 1 are destabilised corresponds to a few exponentiation time-scales, we expect that, after a significant central mass has formed (which takes in $\sim 500 \mathrm{Myr}$ ), a significant fraction of the orbits within the inner few kpc will be destabilised over a few dynamical times (less than a Gyr). In the outer areas this will correspond to a few Gyr. Complete loss of triaxiality and the evolution towards a final state should take about a Hubble time.

\section{Acknowledgments}

Thanks go to Martin Hendry for reading the manuscript. The routine calculating the forces due to order 2 Ferrers bars was kindly made available by Daniel Pfenniger. This work has benefited from many discussions with the 
late Professor R. J. Tayler.

\section{A Equidensity contours}

It is well known (e.g., BT Fig. 2-8) that the logarithmic potential can have wild dents in its density distribution when the axis ratio in one plane is not very near unity. In addition, the density distribution can even be negative. This happens because the potential was chosen for convenience and does not arise from any presupposed realistic density distribution (but actually the reverse is done - the density distribution arises from the potential: This point is discussed in more detail by Pfenniger 1984b who also gives references to the relevant mathematical literature). Nevertheless, the situation is less serious for the three dimensional triaxial potential (as opposed to the flattened axisymmetric one). With two parameters to adjust one can obtain fairly realistic and well behaved density distributions. For example, the diagram on the left hand side of Fig. A.1 shows the equidensity contours for the same model parameters as in the aforementioned BT figure but when $c / a=0.64$. Clearly, although some denting remains, the density distribution exhibits a much better behaviour. Moreover, it was checked that it is always positive. These two properties are connected and it is a general result that triaxial logarithmic haloes for which the axis ratios $b / a$ and $c / a$ are not too different have fairly well behaved density distribution even for significantly asymmetric systems. To see how this happens, we use apply the Poisson's equation to the potential in Eq. (1) and obtain the relation

$$
(p+q-1) x^{2}+p(1+q-p) y^{2}+q(1+p-q) z^{2}=\frac{4 \pi G \rho}{v_{0}^{2}} R_{e}^{2}-R_{0}^{2} P
$$

where $R_{e}^{2}=R_{0}^{2}+x^{2}+p y^{2}+q z^{2}$ and $P=1+p+q$.

If the right hand side and the terms in brackets on the left hand side are positive then this equation can be written in the form of an equation of an ellipsoid with variable axis ratio

$$
\frac{x^{2}}{A^{2}}+\frac{y^{2}}{B^{2}}+\frac{z^{2}}{C^{2}}=1
$$

with

$$
A^{2}=\frac{\frac{4 \pi G \rho_{e}}{v_{0}^{2}} R_{0}^{2}+R_{e}^{2}-R_{0}^{2} P}{p+q-1}
$$



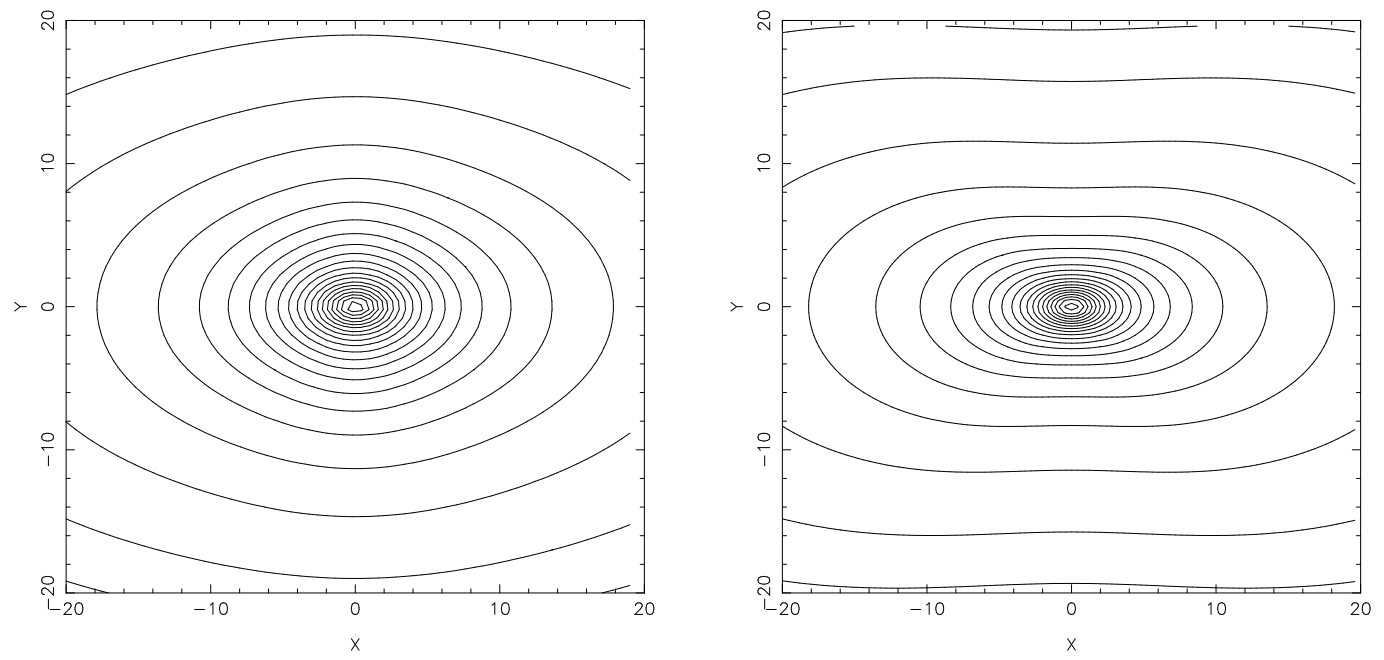

Fig. A.1. Equidensity contours in $x-y$ of Model 1 with prolate halo $(T=0.85)$ and $c / a=0.7$ (left) and when the disk contribution is omitted

Fig. A.2. Three dimensional equidensity contours of some of the systems studied. Top left: Model 3 with oblate halo and $c / a=0.8$. Top right: Model 2 with oblate halo and $c / a=0.8$. Bottom left: Model 3 with prolate halo and $c / a=0.8$. Bottom right: Model 1 with prolate halo and $c / a=0.7$. THIS FIGURE WAS TOO LARGE TO BE INCLUDED

$$
\begin{gathered}
B^{2}=\frac{\frac{4 \pi G \rho_{e}}{v_{0}^{2}} R_{0}^{2}+R_{e}^{2}-R_{0}^{2} P}{p(1+q-p)} \\
C^{2}=\frac{\frac{4 \pi G \rho_{e}}{v_{0}^{2}} R_{0}^{2}+R_{e}^{2}-R_{0}^{2} P}{q(1+p-q)}
\end{gathered}
$$

where $\rho_{e}$ is the value of the density along an equipotential. It is easy to see that

$$
(p+q-1) x^{2}+p(1+q-p) y^{2}+q P z^{2}>0
$$

is the condition for the right hand side of (A.1) to be positive and that

$$
R_{0}^{2} P+(p+q-1) x^{2}+p(1+q-p) y^{2}+q(1+p-q) z^{2}>0
$$

is the condition for the density to be positive. Therefore the conditions for the density to be well behaved - i.e. not being constant on hyperbolic concave isocontours and being positive everywhere - is that

$$
\begin{array}{r}
1+p+q>0 \\
p+q-1>0 \\
p(1+q-p)>0
\end{array}
$$




$$
q(1+p-q)>0
$$

The first two of these conditions are always satisfied while the other two imply that

$$
\|p-q\|<1
$$

This means that one can have a fairly realistic but very flat density distribution using the logarithmic potential as long as the asymmetry is not too different in the principal coordinate planes. However it is clear from (A.3), (A.4) and (A.5) that the equal value surfaces of the right hand side of (A.1) and the equidensity surfaces have axis ratios that differ by factors of

$$
\sqrt{\frac{p(1+q-p)}{1+q-1}}
$$

for $(B / A)$ and

$$
\sqrt{\frac{q(1+p-q)}{1+q-1}}
$$

for $(C / A)$. Therefore for $p$ and $q$ differing significantly from one, the density distribution may be severely dented even when the condition (A.8) is satisfied. This is because the axis ratio will vary significantly along the equidensities. Nevertheless, the equidensities will not be as severely distorted as to be locally hyperbolic (and therefore open) and the density will remain positive.

From the above discussion we expect that the density contours would be better behaved if the potential axis ratios in the plane and normal to it are not too different. For example, the halo equidensity contours even for such a rather extreme potential axis ratio as $b / a=0.7$ are found to have reasonable shape if $c / a \sim 0.64$. This can be seen from Fig. A.1 where have plotted the equidensity contours in the $x-y$ plane of Model 1 with halo potential axis ratio $b / a=0.7$ with and without the disk contribution (the latter case should be compared to Fig. 2-8 of BT where the contours are potted for the case $c / a=1$, in which case they are found to be extremely dented and the density can be negative).

We have also checked the shapes of the three dimensional density distributions by producing some three dimensional contours of this quantity. The relevant contours for several Model parameters are shown in Fig. A.2 


\section{References}

[1] Aarseth S.J., Binney J.J., 1978, MNRAS 185,22

[2] Arnold V.I., 1987, In Mackay R.S., Weiss J.D. (eds) Hamiltonian dynamical systems. J.W. Arrowsmith ltd., Bristol

[3] Aubry S., 1995, Physica D86,284

[4] Barbanis B., Contopoulos G., 1995, A\&A 294,33

[5] Beckman J.E., Varela A.M., Munoz-Tunon C., Vilchez J.M., Cepa J., 1991, A\&A 245, 436

[6] Benettin G., Galgani L., Strelcyn J.M., 1976, Phys. Rev. A14, 2338

[7] Binney J.J. \& Tremaine S., 1987, Galactic dynamics. Princeton Univ. Press, Princeton (BT)

[8] Blitz L., Spergel D.N., 1991, ApJ 370,205

[9] Botteno R., 1996, A\&A 306,345

[10] Burkert A., 1997, To appear in: Klapdor-Kleingrothaus H.V, Ramachers Y. (eds) Aspects of Dark Matter in Astro-and Particle Physics (astro-ph/9703057)

[11] Clifford P.J., 1983, The role of Neutral Hydrogen clouds in the interstellar medium. PhD thesis, Univ. of Sussex

[12] Cole S., Lacey C., 1996, MNRAS 281,716

[13] Combes F., 1991, ARAA 29,195

[14] Combes F., Gerin M., A\&A 150,327

[15] Contopoulos G., 1985, In: Buchler J.R., Perdang J.M., Spiegel E.A. (eds.) Chaos in Astrophysics. Reidel Publishing Company, Dordrecht

[16] Debattista V.P., Sellwood J.A, 1996, To appear in the proceedings of the Dark Matter 1996 conference, Sesto, Italy. Rutgers Astrophysics Preprint Series 194 astro-ph/9610009)

[17] Dubinsky J., 1994, ApJ 431,617

[18] Dubinsky J., Carlberg R., 1991, ApJ 378,496

[19] Eckmann J.P., Ruelle D., 1985, Rev. Mod. Phys. 57, 617

[20] El-Zant A.A., 1997, A \& A in press

[21] Espagnol P., 1995, Phys. Rev. E52,1734

[22] Evans N.W., Collett J.L.,1994, ApJ 420,L70 

412,443

Flores R.A., Primack J.R., 1994, ApJ 427,L1

Franx M., de Zeeuw T., 1992, ApJ 392,L47

Franx M., Illingworth G., de Zeeuw T., 1991, ApJ 383,112

Freeman K.C., 1993, In: Thuan T.X., Balkowski C., Trn Thanh Vn J. (eds) Physics of nearby Galaxies: nature or Nurture? Editions Frontires, Paris

Friedli D., Benz W., 1993, A\&A 268, 65

Gerhard O.E., Binney J., 1985, MNRAS 216,467

Goodman J., Scchwarzschild M., 1981, ApJ 245,1087

Gu Q.S., Liao X.H., Huang J.H. Qu, Q.Y., Su H., 1996, A\&A, in press

[32] Gutzwiller M.C., 1990, Chaos in Classical and Quantum Mechanics. Sringer Verlag, New York

Hasan H., Pfenniger D., Norman C.A., 1993, ApJ 409,91

Hénon M., 1973, A\&A 28,415

Hofner P., Sparke L.S., 1994, ApJ 428,466

Hoogerbrugge P.J., Koelman J.M.V.A., 1992, Europhys. Lett. 19,155

Kandrup H.E., 1994, invited talk at seventh Marcel Grossman meeting July 1994 (astro-ph/9410091)

Katz N., Gunn J.E., 1991, ApJ 377,365

Klafter J., Shlesinger M.F., Zumofen G., 1996, Phyiscs Today Vol. 49 No. 2 P. 33

Klimontovich Yu. L., 1994, Phys. Uspekhi 37,737

Kuijken K., Tremaine S., 1991, In: Sundelius B. (ed) Dynamics of disk galaxies. Göteborg Univ. Press., Göteborg

Kuijken K., Tremaine S., 1994, ApJ 421,178

Kuiken K., Fisher D., Merrifield M.R., 1996, MNRAS 283,543

Lewis J.R., Freeman KC, 1989, AsJ, 97,139

Lichtenberg A.J., Lieberman M.A., 1983, Regular and stochastic motion. Springer, New York (LL)

Lin C.C., Mestel L., Shu F.F., 1965, ApJ 142,1431 
[48] Merritt D., 1996, In: Chaos in gravitational N-body systems, Muzzio J.C. (ed). Kluwer

[49] Merritt D., Fridman T., 1996, ApJ 440,136

[50] Merritt D., Quinlan G., 1997, ApJ Submitted, astro-ph/9709106

[51] Merritt D., Valluri M., 1996, ApJ 471,82

[52] Miyamoto M., Nagai R., 1975, PASJ 27,533

[53] Mulchaey J.S., Regan M.W., 1997, ApJ Letters in press astro$\mathrm{ph} / 9704094)$

[54] Navarro J.F., 1996, astro-ph/9610188

[55] Norman C.A., Ikeuchi S., 1989, ApJ 372,383

[56] Norman C.A., May A., van Albada T.S., 1985, ApJ 296,20

[57] Norman C.A., Sellwood J.A., Hasan H., 1996, ApJ 462,114

[58] Parker T., Chua L.O., 1989, Practical Numerical Algorithms for Chaotic Systems. Springer Verlag, New York

[59] Persic P., Salucci P., Stel F., 1996, MNRAS, in press

[60] Pfenniger D., 1984a, A\&A 134,384

[61] Pfenniger D., 1984b, A\&A, 141,171

[62] Pfenniger D., 1986, A\&A 165,74

[63] Pfenniger 1993, In: Thuan T.X., Balkowski C., Trn Thanh Vn J. (eds) Physics of nearby Galaxies: nature or Nurture? Editions Frontires, Paris

[64] Pfenniger D., Combes F., 1994, A\&A 285,94

[65] Pfenniger D., Norman C.A., 1990, ApJ 363,391

[66] Pfenniger D., Friedli D., 1991, A\&A 252,75

[67] Powell M.J.D., 1968, Atomic Energy Research Establishment Reports: 5947

[68] Quinn T., 1991, Particle simulations of polar rings. In: Warped disks and inclined rings around galaxies. Cambridge University Press, Cambridge

[69] Rix H.-W., 1995, nvited Review at the IAU Symposium 169, "Unsolved Problems of the Milky Way", The Hague, August, 1994 (astro-ph/9501068) 
Rix H.-W., Zaritsky D., 1995, ApJ 447,82

[71] Rix H.-W., Franx M., Fisher D., Illingworth G., 1992, ApJ L5

Roberts M.S., Haynes M.P., 1994, ARAA 32,115

Rubin V.C., Graham J.A., Kenney J.D.P., 1992, ApJ 394,29

Sackett P., Sparke L., 1990, ApJ 361,408

Sackett P., Rix H.W., Jarvis B., Freeman K., 1994 ApJ 436,629

Scalo J.M., Struck-Marcell C., 1984, ApJ 276,60

Schmidt M., 1985, IAU Symposia 106,75

Schwarzschild M., 1993, ApJ 409,563

Sellwood J.A., Wilkinson A., 1993, Rep. on Prog. in Phys. 56,173

Shlesinger M.F., Zaslavsky G.M., Klafter J., 1993, Nature 363,31

Spitzer L., 1978, Physical processes in the interstellar medium. Wiley, New York

Tully R.B., Verheijen M.A.W., 1997, Contibution to ESO workshop November 1996 astro-ph/9702221

Udry S., Pfenniger D., 1988, A\&A 198,135

van den Bergh S., Abraham R.G., Ellis R.S. et al., 1996, AsJ 112,359

van der Kruit P.C., 1986, A\&A 157,230

van der Kruit P.C., 1989, Kinematics and Mass Distributions in Galaxies. In: Gilmore G., King. I.R., van der Kruit P., Buser R. (eds.), 1989, The Milky Way as a galaxy. Geneva Observatory, Sauverny

Verdes-Montenegro L., Bosma A., Athanassoula, 1996, A\&A 306,1011

Warren M.S., Quinn P.J., Salmon J.K., Zurek W.H., 1992, ApJ 399,405

[89] Zaslavsky G.M., 1991, Sagdeev R.Z., Chernikov A.A., Usikov D.A., Weak chaos and quasi-regular patterns. Cambridge University Press, Cambridge 